Portland State University

PDXScholar

Winter 3-21-2016

\title{
Finding the Missing Links: A Comparison of Social Network Analysis Methods
}

Shawn James Mehess

Portland State University

Follow this and additional works at: https://pdxscholar.library.pdx.edu/open_access_etds

Part of the Developmental Psychology Commons, Educational Sociology Commons, and the Social Psychology Commons

Let us know how access to this document benefits you.

\section{Recommended Citation}

Mehess, Shawn James, "Finding the Missing Links: A Comparison of Social Network Analysis Methods" (2016). Dissertations and Theses. Paper 2728.

https://doi.org/10.15760/etd.2724

This Thesis is brought to you for free and open access. It has been accepted for inclusion in Dissertations and Theses by an authorized administrator of PDXScholar. Please contact us if we can make this document more accessible: pdxscholar@pdx.edu. 
Finding the Missing Links: A Comparison of Social Network Analysis Methods

by

Shawn James Mehess

A thesis submitted in partial fulfillment of the requirements for the degree of

\author{
Master of Science \\ in \\ Psychology
}

Thesis Committee:

Thomas Kindermann, Chair

Ellen Skinner

Andrew Mashburn

Portland State University

2016 
Finding The Missing Links, i

\begin{abstract}
Too many students leave school without even the essential skills (ACT, 2011), and many others are so drained by the experience they lack a desire to continue on to a post-secondary education. Academic engagement has emerged as a construct representing students' personal investment in school (Greenwood, Delquadri, \& Hall, 1984), and may be a psychological variable which can be intervened on. However, interventions must occur as quickly as possible to maximize their efficiency (Heckman, 2007). Students' peer groups may be a particularly potent venue of intervention, however several options exist for how to go about measuring their social networks.

In this thesis, social networking data of the only middle school of a small town in the north-eastern United States is analyzed to determine the properties of two collection methods (self-reported networks and participant observations) and four network identification methods (probability scores, reciprocal nominations, factor-analyses, and rule-based). Analyses overwhelmingly supported participant observations as a more inclusive, less biased data collection method than self-reports. Meanwhile, hypothesis tests were somewhat mixed on the most inclusive, least biased network identification method, but after a consideration of the findings and the structural properties of each network, the probability score method was deemed the most useful network. Implications, future research, strengths, and limitations are discussed.
\end{abstract}


Finding The Missing Links, ii

\section{Acknowledgements}

This thesis was written over the course of seven years, and without the help of a small village of loved ones, well-wishers, and interested scholars it may have taken much more. I thank my advisor Thomas Kindermann for believing in me and sticking with me for so long, spending the countless hours typing e-mails and meeting with me to help shape the focus, hypotheses, and analyses. Ellen Skinner has also been an invaluable source of suggestions and alternate takes on many of my assumptions. Andrew Mashburn, for attacking such a lengthy thesis on such a short time-line, but giving it his all and providing wonderful and helpful critiques. Finally, the many conversations I have had with Justin Vollet, Price Johnson, Hyuny Clark-Shim, and James DeLaney have been vital in shaping my approach, hypotheses, and analyses.

Finally, I would like to thank my family. First, my wife, Brittany Vuylsteke, who has stuck it out with me for so long, supporting me even while working on her own Master's degree. Without her help, her understanding, her encouragement, and her ideas, this thesis would surely be a fraction of what it is now. Second, my parents, who were so excited about my thesis, even if they had trouble understanding it, and whose excitement boosted me and kept me going along. Finally, my four-year-old son, James VuylstekeMehess, whose unending love for me inspires me to attempt so much more than I ever dreamed I would, all for him. 


\section{Table of Contents}

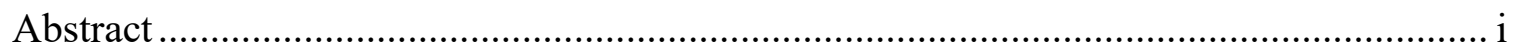

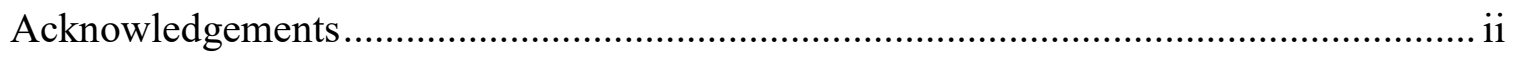

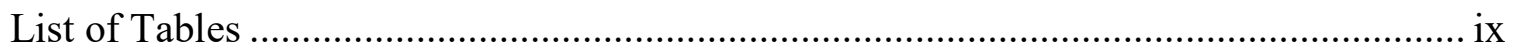

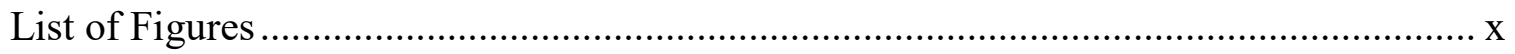

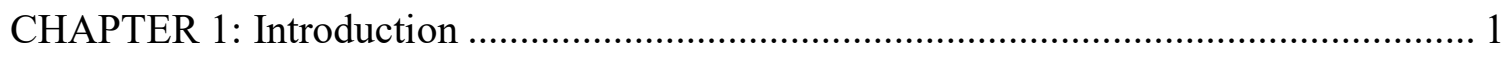

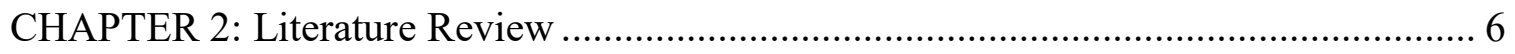

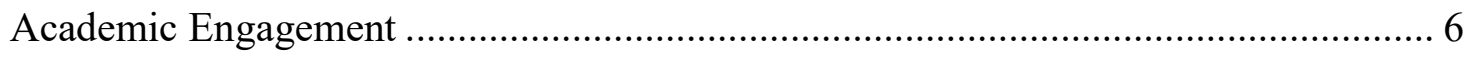

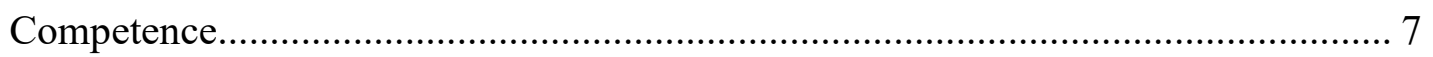

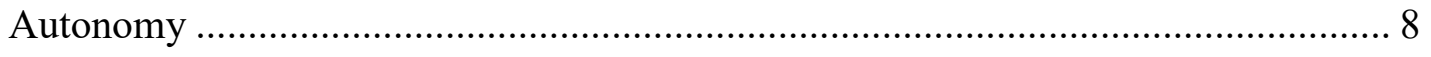

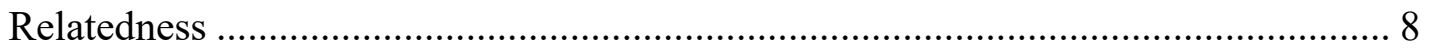

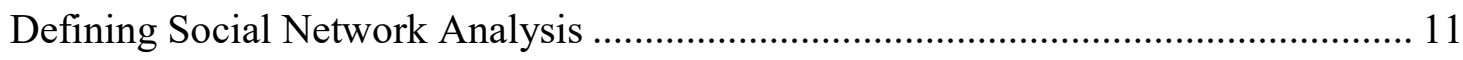

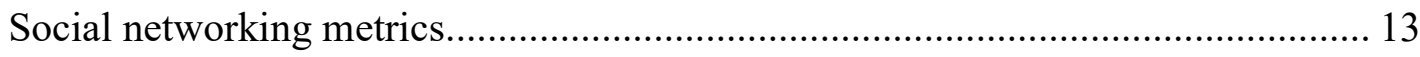

Network Identification Methods ........................................................................... 14

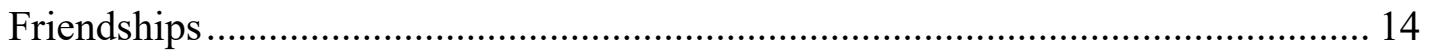

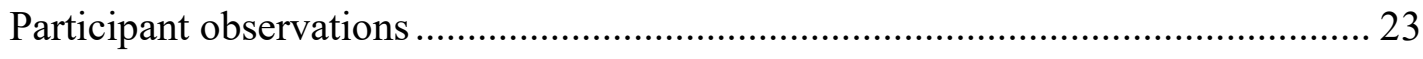

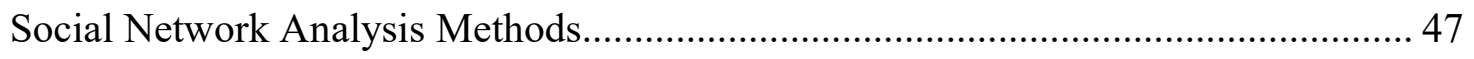

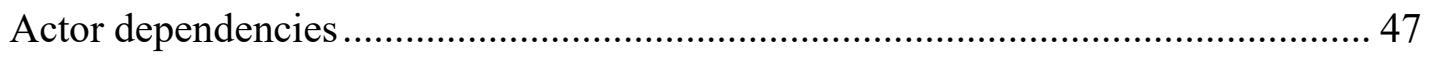

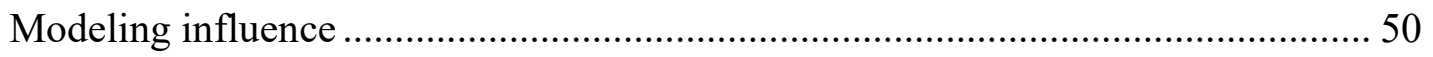

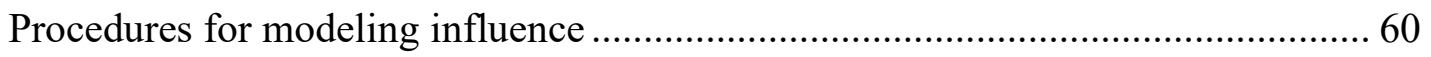


Finding The Missing Links, iv

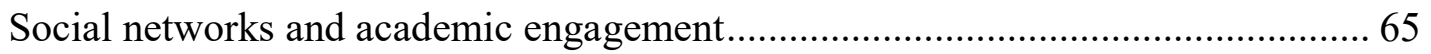

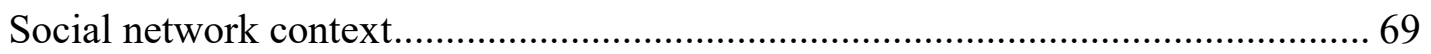

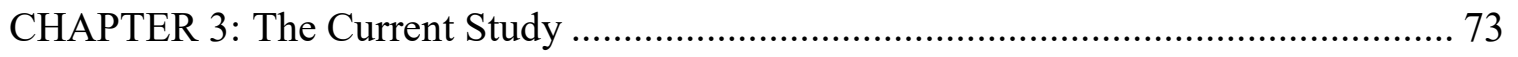

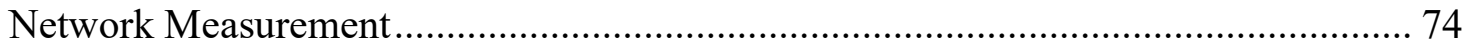

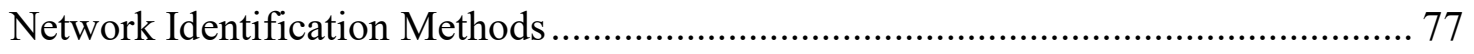

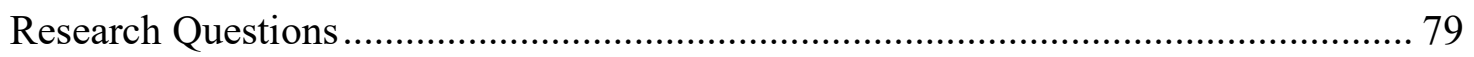

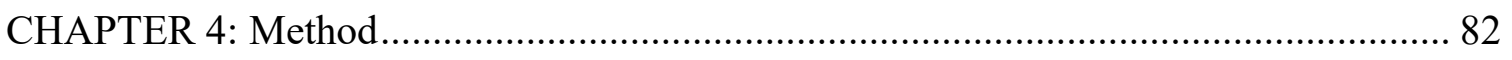

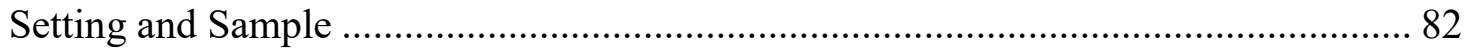

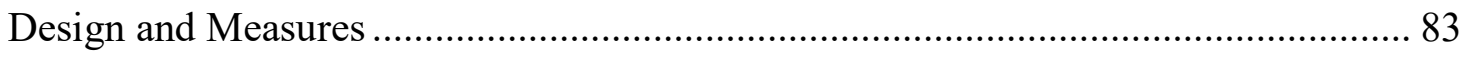

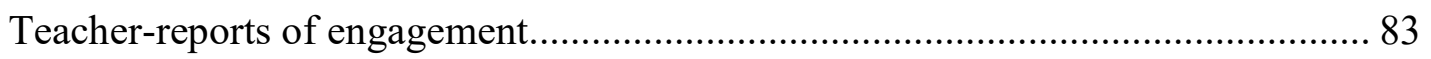

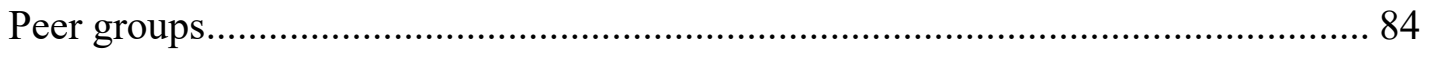

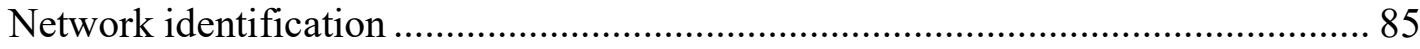

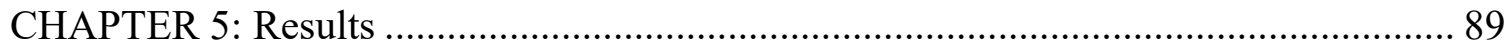

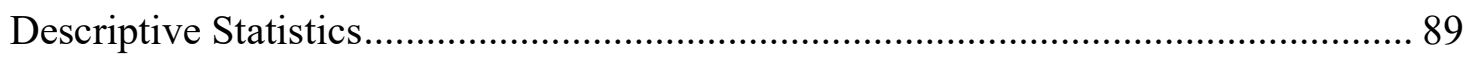

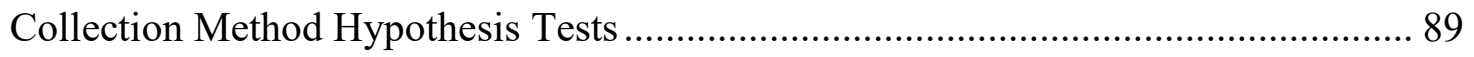

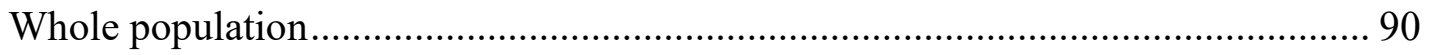

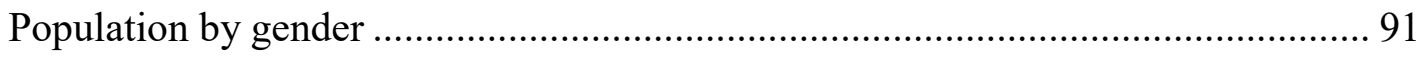

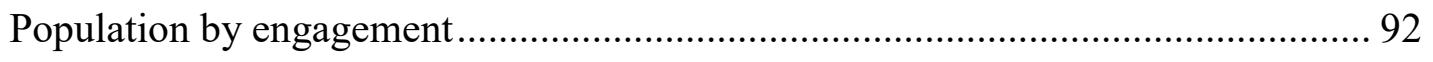

Network Identification Method Hypothesis Tests ................................................. 93

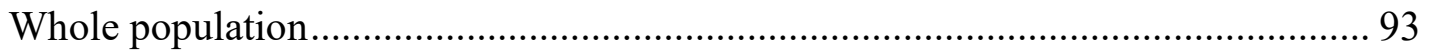


Finding The Missing Links, $\mathrm{v}$

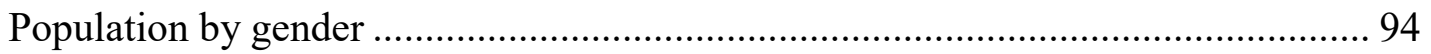

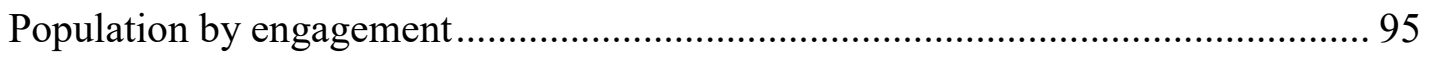

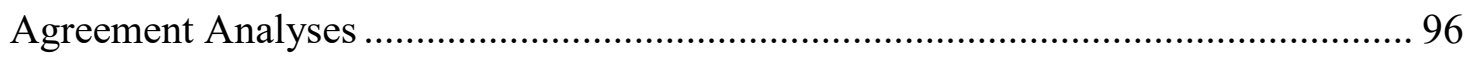

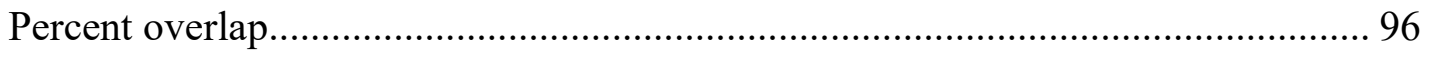

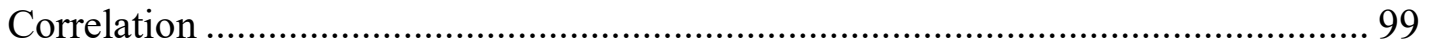

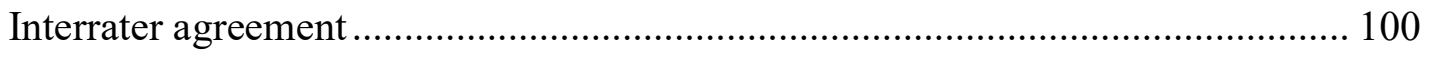

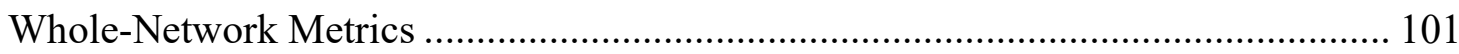

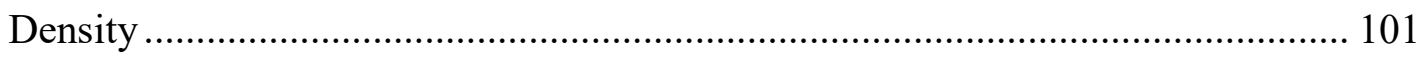

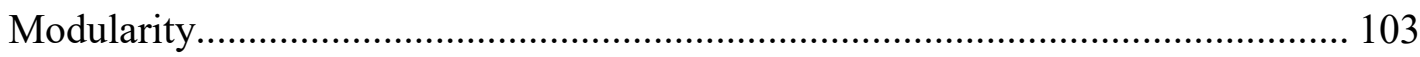

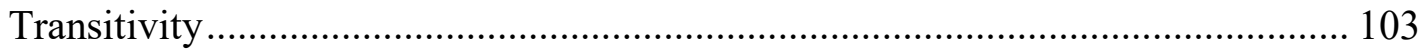

Clustering of academic engagement ........................................................... 104

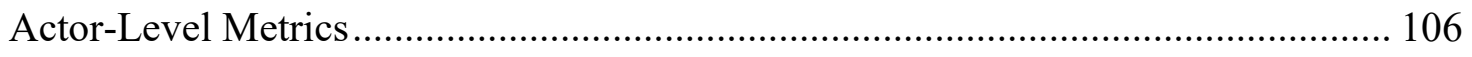

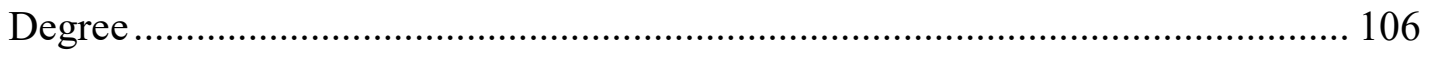

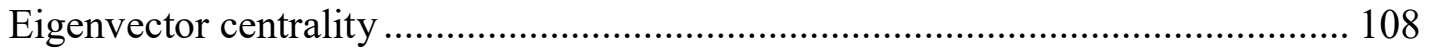

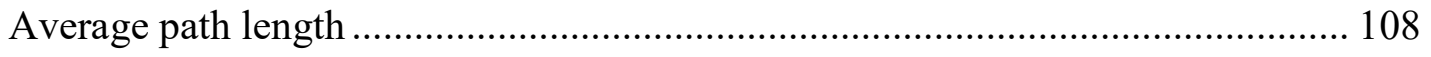

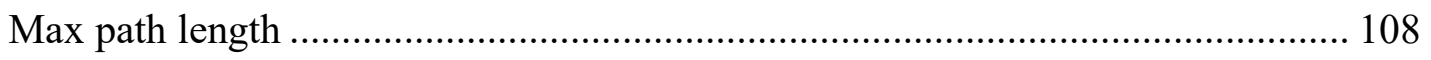

Student-partner correlation of academic engagement...................................... 109

Changes in Academic Engagement by Peer Group ............................................ 109

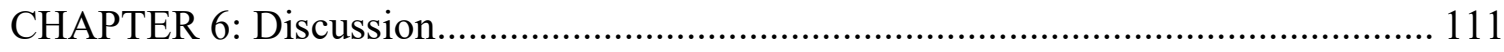


Finding The Missing Links, vi

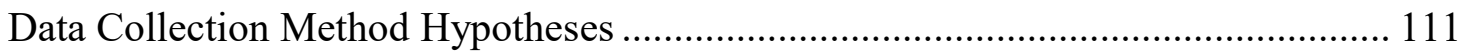

Network Identification Method Hypotheses........................................................ 112

Network Identification Method Properties .......................................................... 118

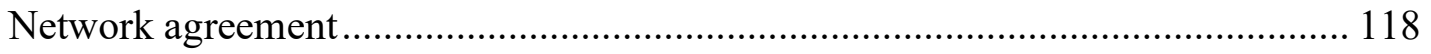

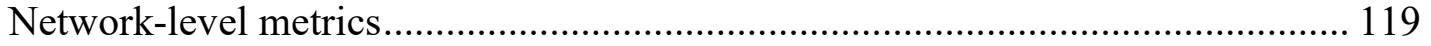

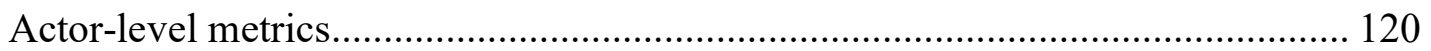

Changes in academic engagement by peer group ....................................... 121

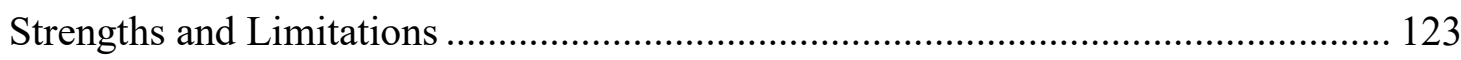

Implications and Future Research............................................................ 125

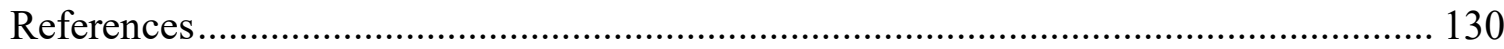

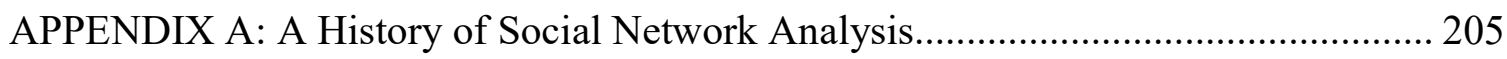

Development of the Social Network Perspective ...................................................... 205

Development of the Social Network Method ............................................................ 207

Graph Theory and Social Network Analysis ................................................... 209

"Who Shall Survive?" and the New Approach.................................................... 210

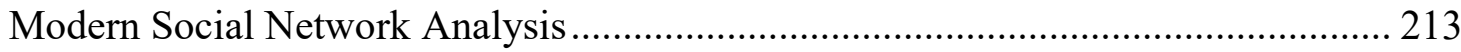

APPENDIX B: Network Data Collection Methods................................................... 214

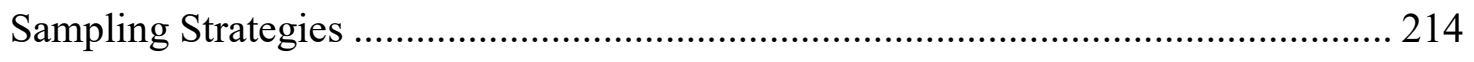

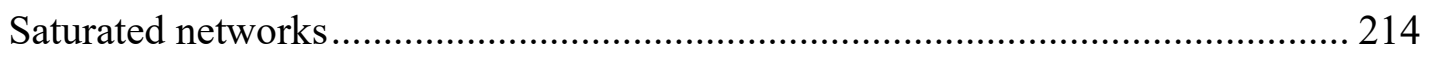

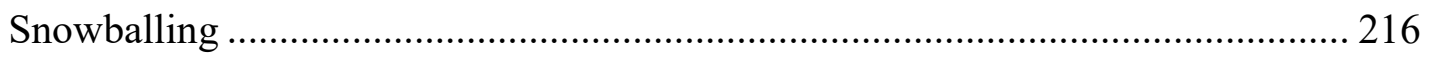




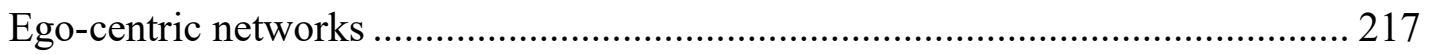

Collection Methods .......................................................................................... 218

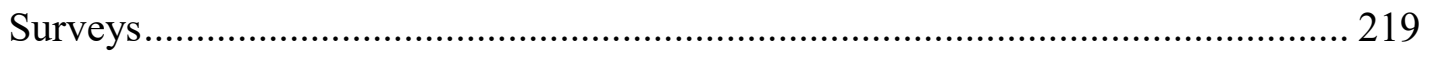

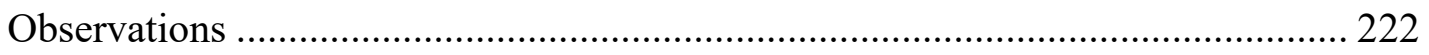

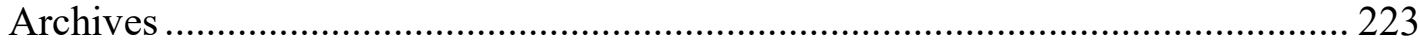

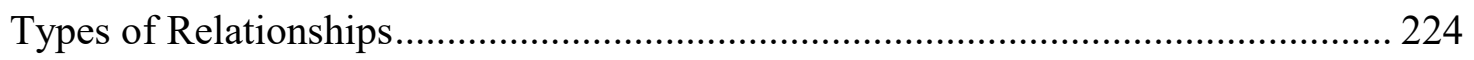

APPENDIX C: Other Network Identification Methods ............................................ 226

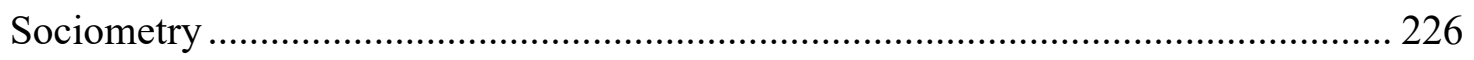

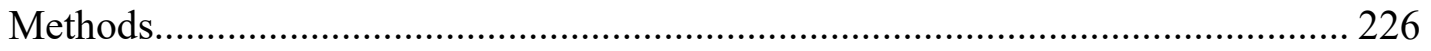

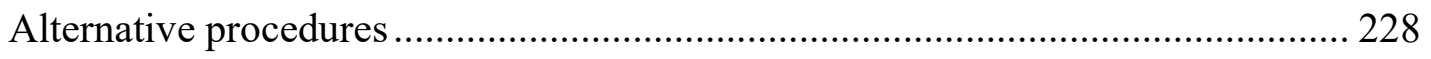

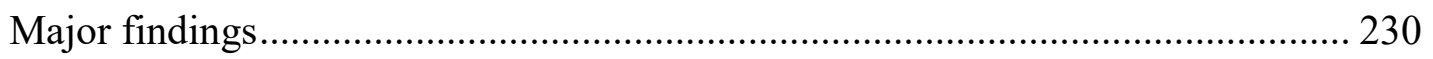

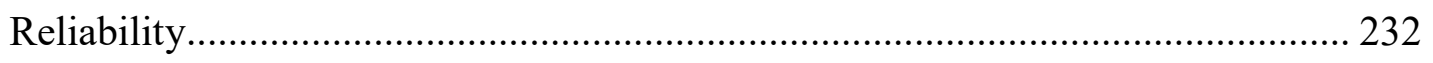

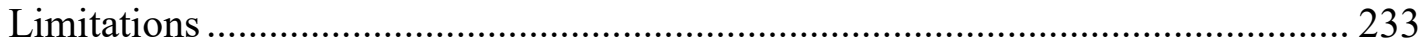

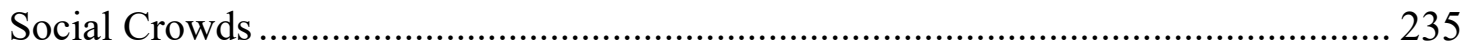

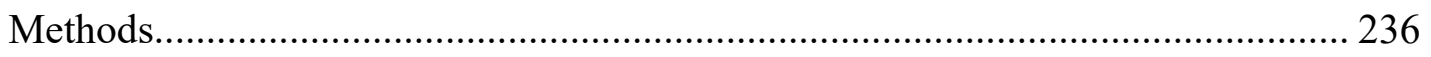

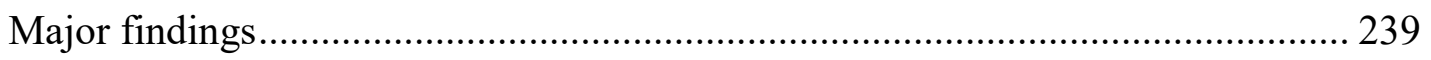

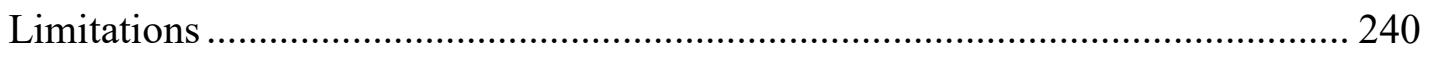

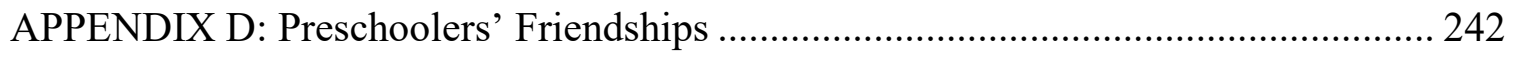

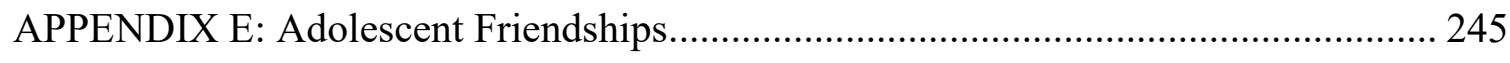

Romantic Relationships ................................................................................ 245 
Finding The Missing Links, viii

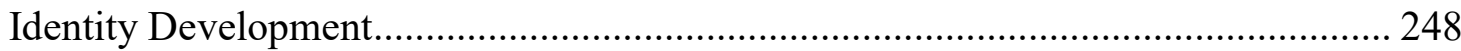

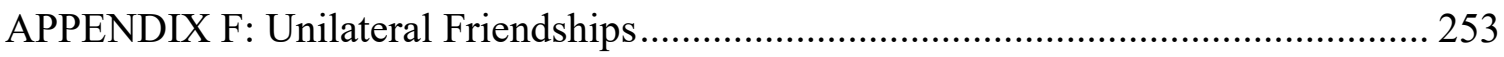

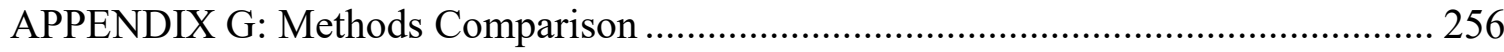

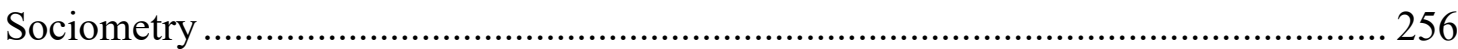

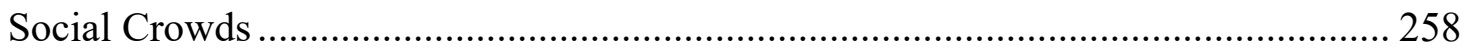

APPENDIX H: Controlling for Network Dependencies .......................................... 260

APPENDIX I: Stochastic Actor-Based Modeling ...................................................... 263 
List of Tables

Table 1. Descriptive statistics for collection methods 90

Table 2. Descriptive statistics for network identification methods 90

Table 3. Log-odds of being covered, by data collection method 91

Table 4. Log-odds of being covered, by data collection method and student gender .......91

Table 5. Log-odds of being covered, by data collection method and academic engagement level

Table 6. Log-odds of being captured, by network identification method 93

Table 7. Log-odds of being captured, by network identification method and student

gender

Table 8. Log-odds of being captured, by network identification method and academic engagement level 96

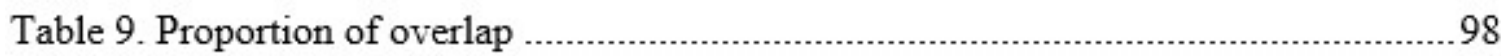

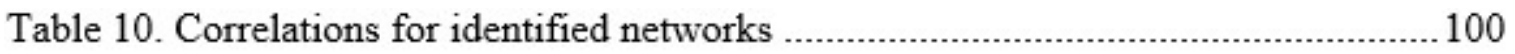

Table 11. Interrater agreements for identified networks ......................................... 101

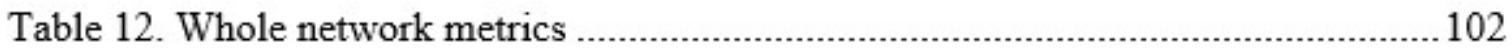

Table 13. Effect sizes (Cohen's $d$ ) of pairwise comparisons of whole network metrics by identified network. 102

Table 14. Actor-level network metrics 107

Table 15. Estimates of selection and influence by network identification method .110 
Finding The Missing Links, $\mathrm{x}$

\section{List of Figures}

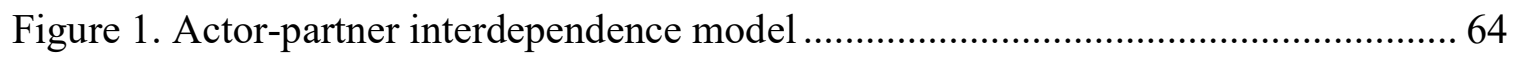

Figure 2. An example of a typical student report....................................................... 85 
Finding The Missing Links, 1

CHAPTER 1: Introduction

The middle school years are an integral period for American students regarding achievements in science and mathematics. Courses in science and mathematics are sequential, and so access to, and success in, later advanced courses in high school and beyond are contingent upon early mastery (Singh, Granville, \& Dika, 2002).

Furthermore, high school curricular choices are determined by performance in these subjects in middle school. In turn, these curricular choices may further influence access to occupational or postsecondary opportunities (Reynolds, 1991). In addition, student achievement has been linked to a series of social, psychological, and behavioral outcomes, such that academic success is associated with development of social competence, engagement in prosocial behavior (Ma, Shek, Cheung, \& Lee, 1996; Masten et al., 1995). Furthermore, academic failure is related to antisocial or delinquent behavior by students (Cheung, 1997; Patterson, DeBaryshe, \& Ramsey, 1989).

Unfortunately, grade school students in the United States are not doing very well. In the 2008-2009 school year, $24.5 \%$ of high school students failed to graduate on time (Aud et al., 2012). Even amongst graduates, reading, writing, and math abilities are poor, with less than one third of graduates able to perform any of these to a satisfactory level, a figure which has decreased since 1992. As noted by The Organization for Economic Cooperation and Development (OECD, 2010), the international market depends on a highly educated workforce, and the United States' drought of highly capable workers may undermine its performance on the global stage. 
Finding The Missing Links, 2

The United States (U.S.) education system is falling behind other countries. According to the OECD (2010), the U.S. was ranked 31 out of 65 countries for mathematics scores in 2009 , falling statistically significantly below the average. Somewhat more encouraging, the U.S. was ranked 23 out of 65 for science scores and 21 out of 65 for reading scores in 2009 , both of which were not significantly different from the average. However, the U.S. was not producing as many high achieving students as many other countries.

According to OECD scores (2010), high achieving students were those who attained Levels 5 and 6 on the Programme for International Science Assessment science scale, Level 5 on the reading scale, and Levels 5 and 6 on the mathematics scale. In 2006, $9.1 \%$ of students in the U.S. attained high achievement on the science scale, not significantly different from the global average of $9.0 \%$. However, less than $.1 \%$ of students attained high achievement on the reading scale (global average was $8.6 \%$ ) and $7.6 \%$ on the mathematics scale (global average was $13.4 \%$ ). The U.S. ranked 18 out of 37 countries for proportion of high achieving science students, 37 out of 37 for high achieving reading students, and 26 out of 37 for high achieving mathematics students.

Academic engagement is a promising construct to intervene on for improving performance in science and mathematics. Previous research indicates higher levels of engagement are associated with increases in academic achievement, adjustment to school, and future success, particularly in industrialized societies such as the U.S. (Luster \& McAdoo, 1994; Ricard, Miller, \& Heffer, 1995; Stipek \& Hoffman, 1980) and Hong Kong (Choi, 2000; Ma, Shek, Cheung, \& Lam, 2000). In the literature, academic 
Finding The Missing Links, 3

engagement refers to processes concerned with education that students engage in (Chen, 2005).

A great deal of research has investigated the precedents of engagement, yet in spite of the inherently social nature of public school, little research has examined the contributions of a child's social network to her engagement. Nonetheless, there is plenty of evidence to suggest this might be a relevant factor. For instance, friendships have been shown to be related to academic performance (Altermatt \& Pomerantz, 2003; Berndt, Hawkins, \& Jiao, 1999; Wentzel, McNamara-Barry, \& Caldwell, 2004) and a deterrent to behavioral problems at school (Ennett \& Bauman, 1994; Poulin, Dishion, \& Haas, 1999). Acceptance scores and sociometric categories have also been correlated with school performance (Chen, Chang, \& He, 2003; Guay, Boivin, \& Hodges, 1999). A few studies have examined the contribution of peers to student engagement, typically finding an influential relationship (Brown, 1999; Kiesner, Poulin, \& Nicotra, 2003; Kindermann, 2007; Kurdek \& Sinclair, 2000; Wentzel \& Caldwell, 1997).

Peers represent a particularly intriguing venue of student influence, as they transcend both the school and home environment, especially in the cyber age (Junco, 2012; Junco, Heiberger, \& Loken, 2011), even though teacher-parent relations may represent a second multi-environment influence on children's engagement (Hughes \& Kwok, 2007). Some researchers have already seized upon the student social environment as a stage for interventions. For example, in a study concerning health behaviors, Valente, Hoffman, Ritt-Olson, Lichtman, and Johnson (2003) used social networking methods in sixth grade classrooms to identify student opinion leaders. A tobacco 
Finding The Missing Links, 4

prevention program utilizing these leaders was found to be more effective at reducing intention to smoke and improving self-efficacy than a prevention program utilizing random students.

To measure the influence of the peer group on individual students' academic engagement, researchers must turn to the field of social network analysis. However, as new methods for measuring and analyzing social networks emerge (e.g., Cairns, Perrin, \& Cairns, 1985), researchers must consider the best method to capture the most at-risk children and to identify their peer groups as accurately and completely as possible in order to properly assess the influence of that group.

In this thesis I will compare the characteristics of two major social network data collection methods: self-reports and participant observations. In addition, I will compare the characteristics of networks identified using four different methods: reciprocally nominated friendships, networks with ties based on probability scores, networks derived from factor analytic methods, and networks based on certain rules and cutpoints. I will argue that data collection methods based on self-reports are inherently biased against boys and students with low academic engagement, and that methodologies based on participant observations will be less biased. In addition, of the four network identification methods, I will argue that networks based on probability scores will be the least biased against boys and students with low academic engagement.

I will begin by discussing the promise of academic engagement as a construct to intervene on to improve student achievement. Then, I will briefly discuss the history of 
Finding The Missing Links, 5 social network analysis and major topics in the measurement and analysis of school-wide social networks before discussing the present study and findings. 
Finding The Missing Links, 6

CHAPTER 2: Literature Review

\section{Academic Engagement}

Student academic engagement is an important goal for schools (Elmore, 1990). Students who are engaged in their education tend to demonstrate greater levels of academic achievement than students who are disaffected (Fredericks, Blumenfeld, \& Paris, 2004; Voelkl, 1997). In addition, disaffected students show a pattern of maladaptive behavior, such as absenteeism, poor overall attitude about school, and greater number of discipline referrals, all of which eventually leads to dropping out (Finn, 1993). These effects have been shown to be stronger for minority students (Voelkl, 1997). A study of high school students in New York (Martin, Fergus, \& Noguera, 2010) revealed that "behavioral engagement... is the greatest predictor of positive achievement among the Black and Latino boys attending these schools" (p. 4), accounting for 12.3\% of the variation in students' grades.

Academic engagement "implies both affective and behavioral participation in the learning experience" (Marks, 2000, p. 155), that students feel and intellectually recognize that learning is important and something they want. Engagement grew out of early attempts to identify behaviors associated with success in school, in which trained observers coded student behaviors into global categories defined ahead of time, such as "on-task", "attending", or "engaged", and compared the frequency of these behaviors to student achievement outcomes (Greenwood, Peterson, \& Sideridis, 1995). These global categories were found to be somewhat predictive of student success. Later research examined individual behaviors, such as "hand raised" or "reading orally", apart from 
Finding The Missing Links, 7

these global behaviors, and found that some were more strongly predictive of student success than the global categories (Cobb, 1972). Intervention studies even found that student success could be improved simply by increasing the frequency of these behaviors (Greenwood et al., 1979; Hops \& Cobb, 1973). Eventually, this research grew to reveal three classes of behaviors: those positively related to academic achievement (e.g., reading silently), those negatively related (e.g., looking at other students) and neutral behaviors (e.g., looking at the teacher).

Today, researchers conceptualize engagement as a motivational process, and thus have turned work toward discovering what will foster motivation and academic growth. One very promising avenue is Ryan and Deci's self-determination theory (2000). The first tenet of self-determination theory is that intrinsic motivation is essential to human functioning: "Perhaps no single phenomenon reflects the positive potential of human nature as much as intrinsic motivation, the inherent tendency to seek out novelty and challenges, to extend and exercise one's capacities, to explore, and to learn." (Ryan \& Deci, 2000, p. 70). The authors go on to note that for intrinsic motivation to flourish, three important supports are required: competence, autonomy and relatedness.

Competence. Competence refers to a feeling that one is effective in what they try to do. Ryan and Deci found that, when feelings of competence were supported, such as in "optimal challenges, effectance-promoting feedback, and freedom from demeaning evaluations" (2000, p. 74), intrinsic motivation was facilitated. Competence has been shown to be important for student success (Pajares \& Schunk, 2001; Schunk \& Pajares, 2005) and adjustment to the middle-school transition (Simons-Morton \& Crump, 2003). 
Finding The Missing Links, 8

Part of the reason for this may be a self-fulfilling prophecy: individuals who believe they can succeed tend to behave as though they will succeed (Bandura, 1997). Competence is best supported when students receive regular and prompt feedback for class- and homework (Pastorelli et al., 2001) as well as personal experiences of success and failure, vicarious experiences of classmates' successes and failures, and verbal persuasion and encouragement from teachers and peers (Pajares, 1996).

Autonomy. Autonomy refers to a feeling that behavior is determined by the self (Ryan \& Deci, 2000) and allows for students to manage their own education in a deliberate way (Doll, Spies, LeClair, Kurien, \& Foley, 2010). When students feel autonomous, they value academic progress (Assor, Kaplan, \& Roth, 2002), set ambitious and useful goals for themselves, regularly act in accordance with these goals, and devote appropriate amounts of time and energy to reach these goals (Masten et al., 1999). The classrooms that are most conducive to students' autonomy are those which tend to set mastery goals to become competent at a particular skill or set of skills, instead of performance goals to outperform other classmates (Pajares \& Schunk, 2001; Turner et al., 2002).

Relatedness. Relatedness refers to a sense of belonging to, and being supported by, others. Relationships are secure and can be counted on to be positive. These others do not necessarily even need to be proximal as "many intrinsically motivated behaviors are happily performed in isolation suggesting that proximal relational supports may not be necessary for intrinsic motivation" (Ryan \& Deci, 2000, p. 75). For school students, there 
Finding The Missing Links, 9

are three contexts important for relatedness: school (teachers in particular), family and peers (Sinclair, Christenson, Lehr, \& Reschly-Anderson, 2003).

Teacher-student relationships have been shown to affect students' behavioral outcomes (Hamre \& Pianta, 2001) as well as social outcomes (Pianta \& Stuhlman, 2004). These relationships are similar to the attachment bonds between parent and child (Kesner, 2000; Pianta, 1999) and is thought to work on at least two levels by making students more committed to their education and by improving their ability to cope with adverse events in their lives (Doll et al., 2010). Both sides of the relationship can work to strengthen it (Greenberg, Kusche, Cook, \& Quamma, 1995). Teachers can show caring, warmth and can offer assistance; they can model fair, responsible and level-headed behavior; they can be open and truthful; appropriately use humor; and encourage and empower student autonomy (Breckelmans, Wubbels, \& Levy, 1993; Damon, 1992; Welker, 1977; Wentzel, 2002). Meanwhile, students can help strengthen the relationship by learning emotional regulation strategies (Greenberg et al., 1995).

Parents are another widely supported source of academic support for students (e.g., Carter \& Wojtkiewicz, 2000; Eccles, Jacobs, \& Harold, 1990). Parental involvement has been shown to positively affect adolescents' grades and mathematics test scores (Muller, 1998) and time spent on homework (Fehrmann, Keith, \& Reimers, 1987), and reduce the chances of a student dropping out of high school (Teachman et al., 1996). These findings have also been supported cross-culturally, amongst students in Hong Kong schools (Siu, 1994; Yao, 1985), and seem to be more important amongst students in low socio-economic backgrounds (Dolan, 1993). 
Finding The Missing Links, 10

As a source of relatedness, peers are of particular interest. First, peer relationships embody an extremely wide range of interactions, as they include all relationships students have with their classmates, be they positive or negative, fleeting or ongoing (Doll et al., 2010). Second, peer relationships and friendships provide a large amount of assistance to students, in the form of companionship, assistance, comfort and even by making school more fun (Johnson, Johnson, Buckman, \& Richards, 1998). As such, peer relationships have been shown to be significantly, positively correlated with academic achievement (Coie, Dodge, \& Kupersmidt, 1990; Wentzel \& Caldwell, 1997), partially by making academic activities more interesting and by making students more active participants in the classroom (Malecki \& Elliott, 2002; Wentzel \& Watkins, 2002).

A third interesting feature of peer relationships is their unique overlap with the school and home environment (Ryan, 2001) and the nearly universal rise in prominence that the peer context undergoes as a child enters early adolescence (Brown, 1990). As children age into teenagers, peer relationships become more intense, influential and closer than in childhood (Berndt, 1982). Early adolescents respond in kind by conforming to peers more, followed by a steady decline in conformity in later adolescence (Berndt, 1979; Steinberg \& Silverberg, 1986). Thus, young adolescents may be particularly susceptible to peer group influence, which is a massive context with a wide range of effects.

A final interesting feature of peer relationships is its measurement. As the peer context is so large, it merits a form of measurement different from the measurement of teachers and parents' relationships with students. This analytical strategy has been 
Finding The Missing Links, 11

developed over several centuries by philosophers, researchers, and mathematicians. We will turn toward a brief history of social network analysis, and consider the modern day application of it.

\section{Defining Social Network Analysis}

Social network analysis (SNA) is the study of the pattern of relationships amongst individuals and groups of individuals in populations. SNA implies an inconvenient reality to most statisticians and researchers, that the assumption that elements under study are independent of one another is false. In some instances, this assumption can be justified via random sampling procedures, but in others (such as a census of a population or within a group of people who know one another), this assumption is often held without justification, as members of populations are likely to contain relationships and hierarchies which can influence individuals' responses to researchers. For example, individuals' social networks can influence their mortality (Berkman \& Syme, 1979), the success of business start-ups (Jenssen \& Agreve, 2002), attitudes toward education (Green \& White, 2007), status attainment (Line, 1999) and adoption of innovative technologies (Valente, 1996). This issue is not likely to go away because, although the population is growing larger, the social world is not. When Jeffrey Travers and Stanley Milgram first performed their famous study of the connectivity of individuals in the United States by sending out postcards, they found that people had, on average, 5.5 to six degrees of separation (Travers \& Milgram, 1969). More recent research by Watts and Strogatz (1998), using chain e-mails around the world, has supported the finding of six degrees of separation, 
despite that the global population was double what it was in 1969. The long-ignored assumption of independence is dangerous for research and is not going away. Thus, researchers should embrace the idea that individuals are connected, and measure and control for this connection through the use of SNA methods.

According to Freeman, there are four features which define the field of modern SNA (2004). First, he states that SNA is motivated by an assumption that people are linked by relationships. Second, he states that SNA can only be determined by empirical measurement. Third, SNA draws heavily on graphic representations, most often on maps of people's social networks. Fourth, SNA trades heavily in the use of mathematical/computational models, as SNA involves the creation of graphs, and (as will be discussed later) utilizes work done in graph theory. Although modern SNA is a relatively new field of research, its beginnings reach back far.

SNA has, in a sense, two origins (see Appendix A for a more in-depth treatment on this topic). Its premise that individuals are connected in patterned ties stems from thinking by $19^{\text {th }}$ century sociologists (and possibly earlier). Methods for the collection of systematic empirical data and drawing of network maps joined this work. Meanwhile, the analytical backbone of SNA, the mathematics and statistics that social networks researchers use, originates from graph theory, which stems from work by $18^{\text {th }}$ century mathematicians. Both strains of thought eventually converged in the $20^{\text {th }}$ century to form the modern social network studies. Today, the mathematics of social networks takes the form of formulas (commonly called 'metrics') which measures any of a number of properties of social networks. Several of these metrics have a demonstrated relationship 
with the workings of social networks of elementary school students, and some have even shown an association with academic engagement.

Social networking metrics. As graph theory and sociology continued to mingle, several formulas from graph theory have crossed over into usage by social scientists. These formulas provide a means to measure a certain aspect of the structure of the network, called a 'metric'. An exhaustive review of these metrics is beyond the scope of this section (see Scott [2012] for a recent treatment), but a relationship between many of these metrics and the formation or evolution of student social networks has been shown, sometimes even directly effecting academic engagement. Such metrics will be discussed in the "Social networking context" section of the "Social Network Analysis Methods" unit.

As these metrics are based on the network under study, how a network is defined, measured, and analyzed is an important decision in any social networking research. Several strategies exist for locating participants for a social networking study, such as exhaustively recruiting and measuring an entire population of known individuals, snowball sampling, or examining ego-centric networks (see Appendix B for a discussion). Meanwhile, there are four major types of social networks (so far): sociometric, social crowds, friendships, and participant observations. As the first two types are not involved in this study, interested readers can view Appendix $C$ for a discussion of them. The latter two types of social networks will be reviewed next. 
Finding The Missing Links, 14

\section{Network Identification Methods}

Networks can be broadly divided into three types, based on their basic unit of study: the characteristic-based network is one made up of collections of individuals who have similar sociometric or behavioral characteristics in common, the group-based network is one made up of clusters of individuals within groups, and the traditional dyadic social network is one made up of links between individuals (Kindermann \& Gest, in press). In each network, there is an assumption that the functioning of the individual is related to group membership and that this membership leads to socialization influences (Rubin, Bukowski, \& Parker, 2006). In this unit, group-based and dyadic networks will be discussed. Social networks based on classical sociometric procedures and perceived commonalities in characteristics (social crowds) are not important for this study, and may be optionally reviewed in Appendix C.

Friendships. Within the realm of child and adolescent social networks, no type of relation has received more attention than friendships. The study of friendship easily predates the earliest forms of psychological research on the topic as religious scholars and philosophers from antiquity have expounded on the conceptual and theoretical considerations of friendship (Newcomb \& Bagwell, 1995). Ancient Greek philosophers such as Plato, Aristotle, and the Stoics explored the meaning of friendship, generally describing it as an expansion of the self (Owen, 1996). Indeed, in the Ancient Greek language, the word for lover ("philos") was also used for friend (Tokar, 2009). Meanwhile, Confucius regarded friendship as a sacred relationship built upon 
Finding The Missing Links, 15

benevolence, fidelity and, most importantly, trust (He, 2007). Friendship received empirical interest at least as early as Monroe's (1898) study of desirable traits in chums and Bonser's (1902) wide-ranging study of the development and elements of friendship. However, in the scientific community at large, friendship in children was generally considered unimportant, receiving a brief mention in the first edition of the Manual of Child Psychology (Carmichael, 1946). In the second edition of the manual (Carmichael, 1954), friendship was covered more extensively in the context of children's social development (Anderson \& Anderson, 1954). By the third edition of the manual (Mussen, 1970), friendship seemed to have gone mainstream with seven chapters dealing with children's socialization, one devoted entirely to peer relations, being included.

In the literature today, friendship enjoys a widespread popularity amongst child and adolescent researchers, as well as sociologists. Friendship is generally viewed as a voluntary (Rubin, Bukowski, \& Parker, 1998), dyadic relationship containing "some degree of reciprocity and closeness" (Erdley, Nangle, \& Gold, 1998, p. 62). Friends are also loyal, trusting, and emotionally intimate with each other (Bukowski, Newcomb, \& Hartup, 1989). Friendships are generally, though not always, identified via reciprocally nominated self-reports (i.e. when asked "who are you friends with?" A names B and B names A).

Friendships appear to be a fundamental aspect of nature. In humans, friendships have been observed in a majority of children as young as four (Howes, 1996) and, when a physical friendship is lacking (as is more likely amongst firstborn and only children), is often filled in with an imaginary companion (Gleason, Sebanc, \& Hartup, 2000; Taylor, 
Finding The Missing Links, 16

1999). Friendships are highly important in these children and remains so all across the lifespan (Buhl, 2009), even as time spent with friends decreases during the transition from adolescence to young adulthood (Adams \& Blieszner, 1996), and again during middle adulthood (Hansson \& Carpenter, 1994). Friendships are observed in every culture, where they are identified distinctly from other close relations, such as kin (Krappman, 1996). In addition, friends amongst non-genetically related individuals are also observed in mammals such as apes, hyenas, elephants, dolphins, and lions. For these animals, having friends generally confers several advantages, such as heightened competitiveness and increased likelihood of reproduction for males, and less stress, superior infant survival, and longer life for females (Seyforth \& Cheney, 2012).

In this section, a broad sketch of friendships in children will be drawn, discussing their measurement, then their basic elements and developmental significance. The section will conclude with a discussion of a particular type of friendship relation that may pose a limitation to the field as a whole.

Methods. Sociometric ratings of popularity, rejection, and peer-directed behavior once dominated the study of children's social environments (Hartup, 1983; Hartup W. W., 1970, 1996), but as the prominence of status and group acceptance has declined over the years, there has been a concomitant increase in the study of children's friendships (Bukowski, 2001; Gifford-Smith \& Brownell, 2003). Part of the reason for the lack of initial enthusiasm in friendships may be due to greater difficulties in operationalizing them and studying them empirically. After all, a friendship means whatever it means to 
the friends in it. Some friends value closeness and honesty, others value some level of mocking and competition.

Hence, for some time, the primary method for identifying friends was via methods inspired from sociometry: peer nominations and peer ratings (Yugar \& Shapiro, 2001). In the peer nomination procedure, children are asked to nominate some certain number of peers on one or more dimensions. Usually, these nominations go toward that child's best friends or others they enjoyed playing with (McConnell \& Odom, 1986). From these lists, positive and negative nominations can be pulled out and used to classify children as being liked, disliked, neglected, and controversial (Coie, Dodge, \& Coppotelli, 1982).

Peer nominations have been subject to criticisms. First, the popular interpretation of these nominations in the literature has been that they provide information on popularity, not friendships (Furman, 1996). In addition, due to fears that there may be negative ramifications in asking students to nominate students they dislike, teachers and parents are often resistant in providing permission, and researchers often limit their nominations only to positives, which may limit the interpretability of the data (Landau \& Milich, 1990; Merrill, 1999). As discussed in more depth in the sociometry section in the appendices, these fears are so far unsupported.

Peer ratings are similar to peer nominations, except that ratings are provided for all peers on some dimension (Asher, Singleton, Tinsley, \& Hymel, 1979; Connolly, 1983). This procedure carries the advantages of a rating for each child (Hintze, Stoner, \& Bull, 2000), so children cannot forget/neglect their classmates, resulting in more reliable and stable instruments (Asher \& Hymel, 1981) and greater sensitivity to changes in social 
Finding The Missing Links, 18

status (McConnell \& Odom, 1986). However, compared to peer nominations, peer ratings can be lengthy to administer, and less appropriate with younger children or children with lower cognitive abilities, as they often assign each child the same score (Odom \& DuBose, 1981). In addition, this method tells nothing of the actual relationships formed among children (Farmer \& Cairns, 1991), making determination of membership and position in specific social groups impossible (Cairns et al., 1985).

The third and currently most popular method for assessing friendships is that of reciprocal nominations. In this procedure, children are who asked to nominate peer who they consider to be their "best friends", using their number of reciprocated nominations as the outcome measure. This method has proven useful for children in kindergarten (Ladd, 1990), elementary school and middle school (Parker \& Asher, 1993), and has even been adapted for preschoolers (Gershman \& Hayes, 1983). Although straight-forward and brief, this method suffers from validity concerns over unilateral nominations (when a child nominates another as a friend but the target fails to nominate in return; Hundley \& Cohen, 1999), as is discussed in more detail in Appendix F.

The fourth method is socio-cognitive mapping (Cairns et al., 1985), in which individual children act as participant observers and are asked by researchers to identify groups of peers whom they observe to "hang around" together (Cairns, Leung, Buchanan, \& Cairns, 1995). Reports from several students can be aggregated, indicating a level of consensus for any pairing of students. Socio-cognitive mapping is still a growing field, but a deep one with valuable insights, and an in-depth discussion of it will be given in the next section of this methodology unit. 
Finding The Missing Links, 19

Benefits and risks. Among preschoolers and young children, healthy friendships are associated with more positive feelings about school, increased participation, and greater levels of achievement (Denham, 2006). In their review, Raver and Knitzer (2002) integrated a few key findings concerning the importance of friends and the ability to make them (termed social-emotional competence). Their first major finding was that the social-emotional competence of young children predicted their academic performance in the first grade, even holding their cognitive skills and family backgrounds constant. In accordance with this, the authors also found that when children did not have developmentally appropriate emotional and social competencies, they tended to participate less in the classroom and were less accepted by teachers and fellow classmates. As such, teachers provided them with less instruction and positive feedback, while classmates were more likely to ignore them. Other research has shown that friends help each other with the transition from kindergarten to preschool (Ladd, 1990), a period fraught with academic and social challenges to students (Ladd \& Price, 1987). Thus, Raver and Knitzer indicated, for students without friends, liking of school and performance suffered. Ultimately, young children with aggressive and antisocial tendencies, and who lacked healthy friendships, were more likely to be held back as a result of their poor performance, ultimately increasing their likelihood of dropping out and persisting in their antisocial behavior (2002).

Outside of school, friends can serve as important sources of social support for children in dire situations. Adult adjustment from childhood maltreatment is significantly enhanced by peer social support (Hyman, Gold, \& Cott, 2003; Runtz \& Schallow, 1997; 
Finding The Missing Links, 20

Tremblay, Hébert, \& Piché, 1999). Lamis, Wilson, King, and Kaslow (2014) found that friends were as helpful as supportive family members in the functioning of AfricanAmerican children suffering from emotional and physical abuse (though not sexual). Friends were similarly important for the attenuation of anxiety symptoms amongst African-American children exposed to community violence (Hill, Levermore, Twaite, \& Jones, 1996). Support-seeking and social-seeking of friends have been found to be among the most commonly employed coping mechanisms for young children (Skinner \& Zimmer-Gembeck, 2007).

Although friendships are generally regarded as a social good (Berndt, 1992), they are nonetheless important for development, which means they can sometimes lead to problems (Sebanc, 2003). In studies of older children and adolescents, friendships have been shown to sometimes be risk factors for antisocial behavior and substance use, depending on the friends themselves and their interactions (Dishion, Andrews, \& Crosby, 1995; Dishion, Capaldi, Spracken, \& Li, 1995). As friendships can and often do follow patterns established in preschool, problematic relationships amongst young children can reverberate across the lifespan (Sebanc, 2003).

Potential downsides notwithstanding, friendships are nonetheless an important element of development. Hartup (1992) suggested that friendships provide four functions for development: 1) a context to acquire and elaborate basic social skills, 2) sources of information for knowledge about the self, others, and the world, 3) provision of emotional and cognitive resources (both for recreation and adjustment to life stressors), 
Finding The Missing Links, 21

and 4) a sort of training ground for the development of the mutual regulation and intimacy inherent in future close relationships.

Next, the benefits and features of friendships for preschoolers and older children will be discussed. For reader interest, see Appendix E for a discussion on the importance of friendship for adolescents, focusing particularly on romantic relations and identity development.

Friendship features. Friendship interests tend to emerge in preschool-aged children, who show interests in superficial traits (Drewry \& Clark, 1985). Gender preferences arrive very early (LaFreniere, Strayer, \& Gauthier, 1984), while racial preferences tend to be much weaker or non-existent (Kinzler \& Spelke, 2011). Friendships are generally centered on shared activities (Bigelow, 1977). For more information about the friendships and preferences of very young children, see Appendix D.

Older, school-aged children show significant changes in their relationships relative to toddlers and preschoolers. Whereas preschoolers tend to befriend one or two others (Hartup, Laursen, Stewart, \& Eastenson, 1988), older children are more likely to have around five friends (Hallinan, 1980). In addition, these friendships show deeper levels of connection.

At this age, older children are more likely to define their friendships around trust and empathy (Bigelow, 1977) and to identify particular personality traits as their reason for being friends with a particular child (Selman, 1980). Influence also tends to take place on a deeper level for older children, with peer talk playing a larger role in child 
Finding The Missing Links, 22

socialization, transmitting cultural categories and norms via elaborate games and rituals, enforcement of criteria for inclusion to the peer group, peer conflict talk, peer group identities, and through resistance of adult culture (Kyratzis, 2004).

At these ages, children show clear preferences for same-gender friendships and cross-gender friendships are less commonly found than amongst preschoolers (Graham \& Cohen, 1997; Howes \& Phillipsen, 1992). Differences in friendship networks are also found, with boys generally engaging in rough-and-tumble games with large friendship networks (DiPietro, 1981; Macoby, 1990), and girls focusing more on pretend play emphasizing individual dyads amongst a smaller group of friends (Benenson, 1994; Howes, Unger, \& Matheson, 1991). The increased closeness involved in girls' friendships is in-line with findings that, although girls are no more likely than boys to have a friend, girls are more likely to report having a best friend (Sebanc, Kearns, Hernandez, Galvin, 2007) and that their friendships tend to be more stable (Howes \& Phillipsen, 1992).

These increased preferences are somewhat a result of boys' decreasing responsiveness to girls' attempts at socializing and girls' ensuing formation of same-sex groups (Howes, 1988; Howes \& Phillipsen, 1992). However, these preferences only appear to be a factor for new encounters, as cross-sex friendships established in preschool tend to be maintained (Howes \& Phillipsen, 1992).

In addition, racial preferences emerge. Lee, Howes, and Chamberlin (2007) tracked the complete social network of all 350 children at an elementary school over the course of one school year, via reciprocal friendship nominations. The authors found that 
Finding The Missing Links, 23

in the first and second grades, social groups were around $14 \%$ less ethnically diverse than the grade population, with girls' networks showing significantly more ethnic homogeneity than boys'. Amongst third and fourth graders, the ethnic diversity of girls' networks did not significantly differ from the ethnic diversity of the grades, while boys' networks increased in ethnic homogeneity up to a $31 \%$ difference from the ethnic diversity of their networks to the ethnic diversity of the grades. However, fifth and sixth grade boys and girls showed no significant deviations from the ethnic makeup of their grades. Thus, although racial preferences emerged, and even seemed to increase for males, these preferences ultimately subsided and continued to show weaker effects than gender preferences. The authors noted that $92 \%$ of all of the social groups in the school included cross-ethnic peers, compared to just $11 \%$ for cross-gender peers, indicating that "gender segregation in children's... friendships continues to be more salient than racial segregation" (Lee et al., 2007, p. 340).

Participant observations. Socio-cognitive mapping (SCM) was at the forefront of the wave of peer nomination methods for social networks. SCM was developed as a sort of response to the two dominant social networks analysis methods for the day. On the one hand there was sociometry, which provided no information about the network structure of a social environment. As noted by Cairns and colleagues "[a] major problem has been that the most reliable social group assessments - such as multiple peer ratings of 'likeability' or 'friendliness,' summarized over subjects - typically lose essential information about an individual's membership and position in specific social groups" 
Finding The Missing Links, 24

(1985, p. 340). While on the other hand there was friendship/peer relationship research, which "has typically reduced the study of social relationships to the individual or dyadic level by emphasizing such constructs as 'popularity' and 'friendship'" (Cairns, Xie, Leung, 1998, p. 25). Thus, although it may capture the social network structure (via the potentially problematic requirement of reciprocal nominations, see Appendix F), researchers in this tradition almost completely neglect the study of the actor's position in the global or local structure.

Cairns and colleagues speculated on some reasons for this divide: the study of group-processes is complicated to examine and analyze, and due to computational and statistical limitations, it may have been more attractive for earlier researchers to simplify matters (1998). In addition, the methods may reflect the focus of the researchers. Namely, whereas psychologists prefer to study individuals, sociologists prefer to study societies. The group, an individual component of a society which is, itself, made up of individuals, may fall outside of the comfort zone of researchers in either discipline.

Instead, Cairns and colleagues set out to develop a method to study social networks themselves (his focus was on school children, and is where SCM is still used most widely), but without relying on reciprocal nominations (1985). Instead, they viewed children as ideal reporters of their social network who should be able to provide reasonably convergent observations, which can be aggregated and used to extract an overall network, a sentiment which would be echoed by other researchers years later (Gest, Farmer, Cairns, Xie, 2003; Kindermann, 2007). Thus, children should be able to remark on not just their connections, but the connections of others in their environment. 
Finding The Missing Links, 25

The method and analysis of SCM will be focused on in this section, with evidence for validity considered and key findings highlighted. Two other approaches that take a participant observation approach will also be discussed and compared to SCM. Finally, comparisons of the two approaches will be reviewed and discussed.

Method. In their initial approach to SCM, Cairns and colleagues interviewed middle-school children, asking "[a]re there some people who hang around together a lot? Who are they?" (1985, p. 341) to elicit children's perceptions of groups within their class or school. To gather information about isolates, the researchers also asked children "[a]re there some people who don't hang around with a particular group? Who are they?" (p. 341). Children were encouraged to give as many or as a few names as they wanted, but they were not provided with rosters. In addition, students were asked about the groups they were part of by asking who their close friends were, who they ate lunch with, who they studied with, and who they would invite to a party. In their next study, the researchers only asked children who their best friends were (1988). Later research used questionnaires over interviews (Xie, Cairns, \& Cairns, 1999). Bagwell, Coie, Terry, and Lochman (2000) used an alternate method, in which they only asked students about their own affiliation groups, and not those of their classmates.

SCM contains a number of strengths over the more traditional methods of social network analysis. First, a census is not needed from the population under study to gain adequate network information. Using Monte Carlo simulations, Cairns and Cairns (1994) showed that SCM can produce reliable network structures with only a participation rate of slightly higher than half. Second, more dyads are captured than otherwise might be if 
Finding The Missing Links, 26

participants were asked only about themselves (as participants may neglect to mention others they do not consider themselves close to, despite that they spend a lot of time together). Third, agreement rates can be calculated via kappa indices (Gest et al., 2003), providing a measure of the trustworthiness of the informants (and a possibility for rerunning analyses after removing informants with low agreement scores, although this possibility has yet to be explored).

Cognitive social structures. Krackhardt (1987) developed a method similar to SCM which he called "Cognitive Social Structures". These were developed as a way of resolving the sometimes major inconsistencies between participants' self-reports of interactions and behavioral reports, a major controversy for the time (Bernard, Killworth, \& Sailer, 1979), and one that persists to this day (Bondonio, 1998; Casciaro, Carley, \& Krackhardt, 1999; Johnson, \& Orbach, 2002; Simpson, \& Borch, 2005).

Krackhardt explained that in "all of these arguments... recall is being used as a surrogate for or measure of behavior." (1987, p. 110). That perceptions are being measured as though they were reality. He suggested an alternate way of considering this concern by focusing on the cognitive reconstructions themselves, which may motivate a change in the thinking of researchers in the field: "[f]rom this perspective, the... findings simply constitute evidence that one should not bother collecting behavioral data, since they do such a poor job of capturing the cognitions which live in peoples' heads." (p. 110).

Krackhardt proposed his method of social network analysis, the cognitive social structure. The network structure of any social environment is represented as a set of 
relational statements between all pairs of actors in the given environment. He describes a set of $R$ matrices (one matrix per relation) that take the form $R_{i, j}$, where $R$ is the relationship under study, $i$ is the sender of the relationship, and $j$ is the recipient. $R_{i, j}=1$ when there is a relationship and 0 when there is not.

He added another layer to this rather standard structure by including a third actor (k) who observes a relationship from $i$ to $j$, forming the cognitive social structure. Thus, an individual unit of analysis would be $\mathrm{R}_{\mathrm{i}, \mathrm{j}, \mathrm{k}}$ : a relationship from $i$ to $j$, as observed by $k$. Thus, the overall data structure would be an $\mathrm{R} x \mathrm{~N}$ x N x N matrix. For analysis, Krackhardt suggested taking "slices", such as all relations sent from actor $i$, all relations received by actor $j$, or all perceptions by actor $k$.

He also suggested more sophisticated matrices called "locally aggregated structures", which would allow the entire data structure to be collapsed into a single network based on certain criteria. For example, one could examine a network made up of instances where $i$ and $j$ reciprocally nominated one another as having a relationship (the classic symmetric network, previously remarked upon in the friendship section). Another method would be to use some sort of criteria to extract a "consensus network" from all observations made by third parties. There is no agreed-upon method for this, but several researchers have offered suggestions informed by threshold values (Krackhardt, 1987; Kumbasar, Romney \& Batchelder, 1994), central graphs (Banks \& Carley, 1994), and informant consensus analyses (Batchelder, Kumbasar, \& Boyd, 1997; Romney, Weller, \& Batchelder, 1986). One proposal by Neal (2008), concerning use of binomial probabilities, will be discussed in more depth in the next section on data aggregation. 
Finding The Missing Links, 28

In addition to the advantages of SCM, cognitive social structures gain an additional one by the exhaustive recording of all possible ties: there cannot be a confounding effect of salience (where respondents nominate certain individuals several times simply because they are currently on everyone's mind), because respondents are prompted for all dyads. Prompting for all dyads may also eliminate the risk of a roster effect, in which participants may be more likely to consider the partners of classmates near the beginning of the roster than the end. However, respondent fatigue may bias participants to think about dyads closest to the beginning of the list. These possibilities have yet to be investigated.

Data aggregation. A great deal of information is collected from each student informant. To make it useable, it must be aggregated and the patterns of affiliation must be extracted. The first step in this process is to collapse the student responses to form an actor $\mathrm{x}$ actor co-occurrence matrix, in which each cell represents the number of nominations from other students indicating an affiliation between the two students corresponding to that cell. The second step is to apply any of a number of algorithms that have been used to determine group membership.

In their original paper, Cairns and colleagues did not describe how they determined group membership from their data (1985), however in their subsequent paper (Cairns, Cairns, Neckerman, Gest, \& Gariépy, 1988), they explained that they previously adopted a "decision rule procedure" in which they used "arbitrary standards" (p. 817) to order cases into groups and to judge whether borderline cases belonged one or more social clusters. For their 1988 study, they performed manipulations of the co-occurrence 
Finding The Missing Links, 29

matrix to determine intercorrelations of co-occurrence profiles between students (in other words, they measured how similar two given students' nominations with other students were by correlating their co-occurrences). Then, students were determined to be part of a group when their intercorrelations were significant $(p<.05)$. Students were allowed to be part of multiple groups if consultation with modification indices indicated that freeing the student to load onto more than one group significantly improved model fit, and if this decision was consistent with the co-occurrence matrix. Later, Cairns, Gariépy, and Kindermann (1991) recommended that an individual have a correlation of $r \geq .4$ with at least $50 \%$ of the group members (the correlations do not need to be significant; Farmer, Stuart, Lorch, \& Fields, 1993). Once a group is identified, all students are assumed to be connected together. In this way, the social network consists of maximally connected, distinctly identified groups.

Bagwell and colleagues (2000) presented an alternative approach by determining groups via exploratory factor analysis. Gest and colleagues (2003) employed a variation of the exploratory factor analysis procedure to arrive at non-overlapping groups: when students loaded onto multiple groups, multiple non-overlapping configurations were constructed and subjected to confirmatory factor analysis. The configuration with the best fit was then used for subsequent analyses.

Later, Gest, Moody, and Rulison (2007) adjusted the procedure so that principle components analysis was used to determine the number of social groups, then they performed a varimax rotation to sort students into their groups. Students whose largest loadings on a principle component (interpreted as a social group) was at least .3 were 
Finding The Missing Links, 30

counted as members of that group. As with Cairns and colleagues (1991), all members are assumed to be connected. So, as before, the social network will consist of maximally connected, non-overlapping groups.

Kindermann's (1993) approach is perhaps the simplest and most straightforward: for his study of fourth and fifth graders, he used binomial $z$-scores to determine whether two students were linked, based on their co-occurrences. First, binomial probabilities were calculated, based on the question "Given that this student has a group, how likely are any other students to be found in this group?" These probabilities were compared with the probabilities that these students were even named in any group (i.e., their salience), resulting in $z$-scores. The $z$-scores were then converted to $p$-values on a normal distribution, and significance could be determined using whichever cutoff the researcher felt appropriate (in Kindermann's study, he used $p<.01$ ). When expected cell frequencies are too low (such as, for example, when a student only receives a handful of nominations from her classmates), this method can be prone to inaccuracy (Haberman, 1988), and so Kindermann later amended (2007) his method to use Sterling's approximation of Fisher's exact test for the case of low expected cell frequencies (in addition to $z$-scores. Thus, both methods had to be significant to yield a positive result).

This may be a case in which cognitive social structures have an advantage, because respondents are asked to indicate a tie for each dyad in the sample. Thus, unlike Kindermann, there is no issue of salience to control for. Instead, Neal (2008) explains that individual responses can be aggregated, and the proportion of respondents indicating a tie between a particular dyad can be calculated to build a "consensus structure", 
Finding The Missing Links, 31

consisting of proportions of agreement for each dyad in the sample. Depending on the analysis and the software, the researcher could stop here, and use the values as a sort of weight or "strength" of the relationship. If they need to be dichotomized, Neal recommends using a binomial test to determine the amount of nominations between two actors that would be above chance $(\alpha=.05)$.

Validity. Self-report seems to be the obvious way to collect social network information. Who knows the memberships of groups as well as their members? Thus, shifting to a method wherein social networks are determined not by the individual in them but by a group consensus would seem strange and prone to error. How can we be certain that informants are reporting on real groups, and not merely crowds of similar people? Are these networks stable, or would informants generate entirely new networks at a different time? There is some good evidence that informants can be counted on to yield trustworthy networks, from three sources: teachers, observers, and the network members themselves.

Teachers. Teachers would seem to be an excellent source of student network information. As Gest (2006) writes: "teachers have extensive direct contact with children's school-based peer social networks so that, in principle, they could become familiar with classroom affiliation patterns.” (p. 249). In addition, teachers' classroom management also shapes the social environments of their class both directly and indirectly (Roland \& Galloway, 2002), so they are a part of the social network to a certain extent. 
Finding The Missing Links, 32

Three studies have examined the level of overlap attained by participant observations and teacher-reported networks and have investigated predictors of agreement. Gest (2006) measured the social networks of 219 children, spread out across grades 1, 3, and 5 in classrooms of four elementary schools via standard SCM methods and teacher-report late in the school year (April and May). He found an overall agreement of $72.2 \%$ for social groups and $72.5 \%$ for friendships. However, most of this agreement reflected agreement in non-affiliations (52.7\% and 58.9\%, respectively). Controlling for chance, the teachers' interrater-reliability was $\kappa=.38$ for social groups and $\kappa=.34$ for friendships, which were statistically significant but weak. Teacher-student agreement tended to be better for higher grades (non-significantly for friendships, however). Gest concluded that "moderate teacher-peer agreement likely reflects limits in teachers' knowledge rather than weak peer report measures" (p. 255).

In another measure of teacher-reported networks (Pearl, Leung, Van Acker, Farmer, \& Rodkin, 2007), researchers investigated networks produced by 549 students in 19 fourth-grade and 11 fifth-grade classrooms (spread across seven schools) in the fall and spring semesters. The researchers examined the overlap between student and teacher reports in both directions: how many members teachers reported who students also reported, and how many members students reported who teachers also reported. In the fall semester teachers, on average, reported $48 \%$ of the members in groups that students reported $(20.6 \%$ of groups identified by students were completely found by the teacher's report, $45.2 \%$ were only partially found, and $34.2 \%$ were omitted). Reversing the analyses, students, on average, reported $86 \%$ of the members in groups that teachers 
Finding The Missing Links, 33

reported (with $74.4 \%$ of groups identified by teachers being completely found in the students' report).

In the spring semester, agreement and teacher ability to report groups generally improved. Teachers reported $61 \%$ of groups that students reported $(20.5 \%$ of groups completely agreed, $67.5 \%$ partially agreed) while students reported $91 \%$ of groups that teachers reported (with $79.8 \%$ of groups being completely found). In addition, teachers identified $22 \%$ more groups later in the school year.

Student personality traits (as reported by the teacher) were found to predict group agreement at both time points. Boys' groups were more likely to be reported on by the teacher when the boys in them were popular and "Olympian" (good at sports, good looking, wins a lot). Girls' groups were more likely to be reported by the teacher when the girls were good students, popular, friendly, and Olympian. In addition, at both time points, the salience of the student (as measured by the number of groups they were nominated to) was related to teacher-student agreement (thus, teachers and students were more likely to agree about highly salient students than about students with low salience. For the fall term, the correlation between individual salience and percent agreement was $r=.45$, for spring term, $r=.4$ ). Group size was not related to agreement at either time point. The authors concluded that teachers were effective at assessing the social networks of their students and went on to note that groups that teachers missed also tended to be groups that were less salient to students as well (as measured by nomination frequency).

Finally, another study that examined the social networks of students via cognitive social structures (Neal, Cappella, Wagner, \& Atkins, 2011) generally found more support 
Finding The Missing Links, 34

for the validity of participant observations. Out of 33 second through fourth grade classrooms in poor schools, 32 revealed above chance levels of overlap, with an average agreement of $40 \%$ (ranging between 10\% - 71\%). Agreement was positively predicted by grade and classroom productivity, and negatively by class size, quality of behavioral management, and level of aggressive behavior (altogether accounting for $67 \%$ of the variance in teacher-student agreement). The time of the school year the study was conducted in was not mentioned.

Thus, these three studies provide support for the validity of participant observations by finding overlap (sometimes a great deal) with teacher-reports. The overlap is moderate, as would be expected, as "teachers are privy... to only a sample of children's interactions with one another" (Pearl et al., 2007, p. 26). However, teachers showed higher levels of agreement with students as the year went on, further supporting that teachers' skills were improving, not that they and their students were reporting on two different things.

A concern would be raised if teachers systematically identified groups and connections that students did not, which might indicate that students misunderstood the instructions and misidentified social groups. However, as Pearl and colleagues investigated, this did not seem to happen to a large extent. Gest (2006) and Neal and colleagues (2011) found significant effects of grade on teacher-student agreement, fitting in well with previous studies indicating that student relationships become more stable over time (Cairns et al., 1995; Putallaz \& Wasserman, 1989). These findings should be noted for other researchers investigating social networks in early grades: a high criteria 
Finding The Missing Links, 35

for significance may be needed if they are seeking reliable, and not transitory, connections.

These studies do highlight some of the limitations to using teachers as a sole source of networks: their errors of omission cannot be argued to be randomly distributed, as Pearl and colleagues found while examining student characteristics as a predictor of agreement (2007), and as Neal and colleagues found in examining classroom management (2011). However, as an indicator of the validity of participant observations, they provide helpful support.

Observers. Observers have the advantage over teachers of being a fresh set of eyes, unclouded by past interactions with students, and so they may be able to bring a different perspective to the students' social networks. Two studies have compared the social networks generated by informants to observers' own impressions.

In his seminal paper, Cairns and colleagues (1985) performed observations of the classes they gathered their first SCM data on and found that the students had four times as many positive interactions with ingroup members as they had with outgroup members (there was no significant difference for negative interactions).

Gest and colleagues (2003) selected three boys and three girls from each of three fourth and three seventh grade classrooms to complete a socio-cognitive map of their class. The researchers also performed observations of the classes, in order to generate their own social network of the classes. The correlation between the students' map and the researchers' observations in each classroom was significant, with a mean correlation of $r=.55$. Breaking down results by the number of nominations to social clusters, 
Finding The Missing Links, 36

students with the highest amount (ten or more) tended to show the highest correlations with the observers $(\mathrm{r}=.67, p<.001)$. The correlations declined as the number of nominations for the student fell: students with less than three nominations showed much lower correlations with teacher reports $(\mathrm{r}=.38, p<.05)$.

Unfortunately, neither study reported some measure of percent agreement, so ascertaining the exact level of overlap is difficult. Nonetheless, Cairns' and colleagues findings that students interact more frequently with their ingroup than their outgroup (1985) is helpful, as it is essentially what is implied by asking which students "hang out together". Gest's correlations are encouraging, although difficult to interpret. However, his findings about the number of nominations being related to correlation of the overlap would later be echoed by Pearl and colleagues (2007) finding that student salience helped predict teacher-student agreement.

Students. The students themselves can indicate the validity of the participant observation procedure via information about reliability and their overlap with other selfreport measures of affiliation. These possibilities have been examined in two studies.

Cairns and colleagues (1995) elicited social network information from 132 students in fourth and seventh grade classrooms via their SCM procedure. The researchers measured students, and measured them again three-weeks later to assess the stability of the connections. They found that, using liberal criteria for stability, the groups were fairly stable ( $90 \%$ of groups retained/modified $50 \%$ of their members from time 1 to time 2). Using more conservative criteria, the groups showed much less stability ( $26 \%$ of the groups were unchanged from time 1 to time 2). Self-reported friendships also 
Finding The Missing Links, 37

exhibited a noticeable degree of fluidity (with correlations of $r=.32$ and $r=.46$ for grades 4 and 7 , respectively).

Group centrality was not found to predict group stability, although individual centrality was (in other words, highly central students within a group were more likely to still be with that group at time 2 than more peripheral students). Student centrality also proved to be fairly stable. The researchers divided students into three categories, depending on how many nominations they received for the entire network: the students with the most nominations were called "nuclear", those with the least were "peripheral", while those in between were "secondary." Nuclear students tended to be the most stable, with $76 \%$ fourth grade/ $69 \%$ seventh grade nuclear students from time 1 falling into the same category on time 2 . Peripheral students were much less stable, with stability rates of $58 \% / 60 \%$. Secondary students were close behind, with rates of 55\%/57\% (those that changed were roughly equally likely to be categorized as nuclear or peripheral at time 2).

In a smaller study of 16 students from two classrooms at a residential school for children with emotional and behavioral disorders (Farmer et al., 1993), students' social networks were assessed via SCM and, on a separate occasion only a few days apart, they were asked who their playmates were (assessed by asking students who they performed various activities with, including roller skating, listening to music, and talking). A chisquare analysis revealed that students were significantly more likely to identify playmates from members of their ingroup than from their outgroup.

Cairns and colleagues' (1995) study is helpful in demonstrating that the SCM procedure can produce fairly reliable results. Their findings may have been lessened by 
Finding The Missing Links, 38

their reliance on developing well-defined groups, rather than relying on connections between students (one major criticism of the SCM procedure. See the "Limitations" section of this unit for a discussion). Indeed, the stability of individual centrality was the more impressive finding. Meanwhile, Farmer and colleagues' (1993) findings that playmates and SCM group members were often one and the same suggested that students understood the directions and could identify students based on publicly observable affiliation patterns. Holding the measures on separate days was useful to reduce the likelihood that students' responses to the playmate questionnaire were influenced by their responses to the SCM questions.

Limitations. Cairns and colleagues' (1988) procedure for calculating social groups continues to be one of the most commonly used methods (likely due in no small part to the specialized SCM program; Leung, 1998), enjoying wide use today (Day, Hamm, Lambert, \& Farmer, 2013; Nichols \& White, 2014; Webb \& Zimmer-Gembeck, 2014). However, this method is not without some concerns.

First, whether the method dealt with directly examining a correlation matrix or employing a factor analytic method, the correlation was the mathematical unit of measurement. In this context, a correlation is fundamentally different from a probability score, as the correlation measures similarity between individuals in both who they are associated with and how often. Thus, use of the correlation implies that two people are in a group together when they are associated with the same people. Interestingly, the number of co-occurrences between the students need not be high, or even above zero, to attain a high correlation. This reveals an important limitation in this calculation 
Finding The Missing Links, 39

procedure, as pointed out by Neal and Neal (2013): connections between students who have a high number of co-occurrences, but who are associated with different people (friends from the "opposite sides of the railroad tracks" so to speak), will be missed. As such, the calculation itself reduces the number of overlapping groups that students can be part of, since, by definition, a student who is part of overlapping groups will have a set of co-occurrences that is not particularly close to members of either group (except with other students who overlap with the same groups). This may restrict researchers who are especially interested in students who are in multiple groups, such as liaisons (Ennett \& Bauman, 1993; Henry \& Kobus, 2007; Shrum \& Cheek, 1987). Furthermore, depending on how much their profile differs from the students in either group, the student might be lucky if they are assigned to a group at all. Of course, using an approach such as Gest and colleagues' (2003) to eliminate overlapping groups will only serve to magnify this effect.

In addition, Neal and Neal also charged that the correlations may be miscalculated. Cairns and colleagues (1991) and Gest and colleagues (2007) both filled in the diagonal of the co-occurrence matrix with the number of groups the corresponding actor appeared in and proceeded from there. However, this approach has been criticized as "comparing apples to oranges" (Neal \& Neal, 2013, p. 607) by mixing diagonal and off-diagonal values in the calculation of correlations. Instead, they argue that the diagonals need to be completely disregarded in the calculations.

These points are part of a larger criticism that these methods will tend to simplify the social network by replacing links between dyads with groups where there may be none. Watts (2003) noted that when social networks are analyzed with methods similar to 
Finding The Missing Links, 40

those used in SCM, "even a random ... network—one that has no particular structure built into it at all—will be highly clustered" (p. 128). Neal and Neal (2013) extended this point by demonstrating that, for example, given four students in a class, other student informants might nominate various combinations of some of those four as being in a group together, however none of the informants could nominate all four of them as being in a group together. However, due to the calculation procedure, the four of them may nonetheless be assigned to the same group. As Cairns' goal was to identify and study groups, this may not be particularly troublesome to him, however it poses some questions about the validity of the calculation procedure: have the groups been correctly identified?

Krackhardt's cognitive social structures largely avoid these pitfalls by emphasizing the individual's perception of the social network, and not some objective reality. However, this method is ultimately hindered by one important limitation: the amount of data. As noted by Krackhardt, "getting a respondent to voluntarily record his/her perception of every $(i, j)$ dyad for each relation in a bounded system of actors is a formidable task." (1987, pp. 113-114). As such, this approach may not be practical for large networks, such as entire schools (Neal, 2008). For example, middle schools in the United States have, on average, 575 students (U. S. Department of Education, 2010). A cognitive social structure study would require students to remark on each of 330,625 pairs of students which, at a hypothetical rate of two pairs per second, would require 44 hours' worth of work for a single data point! Thus, unfortunately, cognitive social structures are simply not an option for most schools. One possibility may be to measure the networks of each classroom in a school, however this would nonetheless miss 
Finding The Missing Links, 41

important relationships between students in different classrooms. As such, cognitive social structures have found greater popularity in studies of social networks within businesses (Bolino, Turnley, \& Bloodgood, 2002; Krackhardt \& Kilduff, 2002; Tsai \& Ghoshal, 1998) and individual classrooms (Neal, 2008; Neal et al., 2011), where social environments under study tend to be much smaller.

Kindermann's $z$-scores may be the best option. As they rely on the number of nominations between a given pair of students, and not who else those students have been nominated with, they are largely exempt from the criticisms concerning correlation calculations. Furthermore, Kindermann (2007) argues that naturally existing groups are more complicated than non-overlapping clusters, and suggests using dyadic links between individuals to construct the network, then analyzing who in the network individuals are connected to. Thus, "each member is seen as having his or her own unique network" (p. 1188), a sentiment similar to Krackhardt's. Analyses may still proceed involving groups, but instead of a sort of static group that is the same for each of its members, groups are defined relative to the individual under study. In turn, this should model the structure of the social network more closely than the other methods without the workload of Krackhardt's cognitive social structures. Given these advantages, it is surprising that Kindermann's method has not been adopted or compared to those used more commonly.

\section{Comparison of self-reports with participant observations. Generally,} comparisons of self-reports and participant observations yield relatively similar networks. 
Finding The Missing Links, 42

In one of the earliest comparisons, Cairns and colleagues (1995) found a correlation of $r$ $=.79$ between self-reports and participant observations. Pijl, Koster, Hannink, and Stratingh (2011) reported a similar correlation of $r=.74$. Other researchers used the kappa coefficient, a measure of interrater agreement, to measure similarities in networks. In these studies, agreement was generally small to moderate with $\kappa=.48 / .53$ (fall and spring assessment within one school year, respectively; Rodkin \& Ahn, 2009). Neal and colleagues' (2014) comparison of self-reports with cognitive social structures in 30 classrooms yielded a similar mean classroom level of agreement (mean $\kappa=.49)$. However, high correlations do not necessarily imply that the same individuals and ties have been captured.

Still other researchers simply reported the percentage of overlap. Yugar and Shapiro (2001) reported an exact match between self-reports and participant observations $82.8 \%$ of the time, a partial match $6.3 \%$ of the time, and no match $10.9 \%$ of the time. Neal and colleagues' (2014) work with cognitive social structures revealed an overlap of $80 \%$ when comparing individual respondents' data with self-reports. Her previous work with colleagues involving aggregated respondent data turned up an overlap with reciprocally self-reported ties of $86.7 \%$ (2008) and 49\% (2012). There was no comment about the unusually low overlap in the 2012 study. Finally, Molloy, Gest, and Rulison (2011) compared reciprocally nominated friendships, frequent interaction dyads (similar to the approaches of Kindermann [2007] and Neal [2008]), and social groups (similar to Cairns and colleagues' [1985] approach, though identifying groups via principle components analysis) in fifth and seventh grade students. In their data, 70\% of friends 
Finding The Missing Links, 43

were in the same social group together, while $33 \%$ of social group members were also friends. In addition, $61 \%$ of the time, friends were also frequent interaction partners, while $53 \%$ of frequent interaction partners were friends. Finally, frequent interaction partners showed the largest overlaps with social groups, with $91 \%$ of partners being in the same social group together, and $52 \%$ of social group members also turning up as frequent interaction partners.

A second major result from these studies was the size of the networks. Two of the networks using SCM methodology indicated that networks derived from participant observations were larger than those derived from self-reports. Gest, Moody, and Rulison (2007) reported a density of .49 (indicating that $49 \%$ of all possible ties were present) and between $10-24$ groups (depending on the group identification method used) using participant observations, and a density of .12 and 20-24 groups using self-reports. Rodkin and Ahn (2009) reported 40\% more children per group using SCM than using self-reports, and about one third the amount of isolates (though they also reported 20\% fewer groups per classroom, which may be due to the SCM procedure's limitations with overlapping groups). On the other hand, using cognitive social structures, Neal (2008) reported network densities of .17, versus .21 for self-reported data. Neal and colleagues (2014) similarly reported network densities of .29 and .36 for cognitive social structure and self-report data, respectively.

This discrepancy may be due to the nature of cognitive social structures and how groups were calculated. The SCM studies used procedures designed to generate groups of actors, in which actors inside of the groups were by default assumed to be connected to 
Finding The Missing Links, 44

one another. The cognitive social structure studies, on the other hand, used two different tests designed to identify dyads. Neal (2008) used a binomial test to identify any nominations above chance. This study, which was conducted on 15 children, may have been underpowered to detect whether some of the lower frequency nominations were above chance (as discussed earlier, Kindermann recommends use of Fisher's exact test in the case of low cell frequencies). In addition, although they have never been compared, it would seem reasonable to expect that cognitive social structure techniques, which are based on recognition of pairs, would elicit more dyad nominations than SCM, which is based on free recall of groups. Thus, this may result in a higher level of "noise" (irrelevant dyad nominations), requiring a higher conditional probability (brought about by a higher number of co-nominations) for a binomial test to rule a dyad as being nominated more often than chance.

The second study by Neal and colleagues (2014) used a majority rule for dyads (i.e., if a pair of actors was nominated by at least $50 \%$ of their classmates, they were considered connected). Such a rule may be overly conservative, as indicated by Neal and colleagues' 2008 study, in which an analysis of a different dataset using a majority rule revealed that, while this rule recognized ten of the self-reported relationships, it missed twelve of them (by comparison, the binomial rule recognized thirteen and missed nine). Thus, a small sample size and an improper rule may have led to improperly identified networks that were smaller and less dense than they should have been.

A third major result that these studies can provide is a breakdown of where the disagreements occurred. Cairns and colleagues (1995) reported that there were only a 
Finding The Missing Links, 45

"few" (p. 1340) false negatives (peers missing a self-reported reciprocal relationship) but a "frequent" (p. 1340) amount of false positives (peers putting two students together when neither reported being together), though no numbers were provided. This general finding was reflected by Pijl and colleagues (2011), who found a false negative rate of $20 \%$ and a false positive rate of $48 \%$. Molloy and colleagues (2011) did not report false negative or false positive statistics, however the inverse of some of the overlaps between friends, frequent interaction partners, and social groups, can be taken as false negative and false positive rates. For false negatives, the percent of friends who were not also in the same social group was $30 \%$, while the percent of friends who were not also frequent interaction partners was $39 \%$. For false positives, the percent of social group members who were not also friends was $67 \%$, while the percent of frequent interaction partners who were not also friends was $47 \%$. Finally, comparing partners and social groups, only $9 \%$ of partners were not in the same social group (if using social group to predict frequent interaction partner, this would be a false negative), while $48 \%$ of social group members were not partners (a false positive in the aforementioned scenario).

Critical review. One limitation of all of these comparisons is that they did not specify the composition of their peer networks, relative to the self-report networks. In other words, in none of the studies was it ever described whether students who did not complete a self-report were left as part of participant observation networks. On the one hand, leaving non-responders in participant observation networks is externally valid. On the other hand, the differences in sample composition introduces a confound for 
Finding The Missing Links, 46

comparisons: to what extent are differences between methods due to the methods, versus being due to the differently composed samples?

Another limitation, found in all but two of the comparisons (Cairns et al., 1995; Gest et al., 2007), was the use of within-classroom data. Cairns and colleagues did not comment on the proportion of friendships or ties based on participant observations that were out of classroom (1985), but Gest and colleagues did: they reported that over half of self-reported friends and peers nominated to social groups were from outside of the respondent's own classroom (59.4\% and 51.1\%, respectively; 2007). Kindermann (2007) reported a similar percentage of out-of-classroom participant observations (40\%).

Finally, the use of a multitude of statistics in reporting network similarity renders comparisons between studies difficult. As each statistic reports on a related but different concept, future comparisons would be advised to use all three of the commonly encountered statistics (percent overlap, correlation, interrater agreement) to aid readability.

Nonetheless, in taking these findings together, a clearer picture is drawn of what is measured by self-reports versus participant observations. As would be expected, there is a high concurrence between self-reported and participant observed networks. However, the concurrence is not high enough to suggest that they are one in the same. After all, the standard question for SCM studies is "who do your classmates hang around with?", not "who are your classmates friends with?" Even asking students who they "hang around with", versus who they are "friends with", yields only somewhat overlapping responses (agreement rate of $\kappa=.59 / .70$ for fall and spring terms in one school year; Rodkin $\&$ 
Finding The Missing Links, 47

Ahn, 2009), indicating a clear difference between friends and frequent affiliates in the minds of respondents. It would seem likely that friends are probably considered to be more emotionally intimate than people that one "hangs around" with. This notion is supported by the findings of larger and thicker groups using SCM than self-reports. It makes sense to have a few people one is close to and many one is not.

\section{Social Network Analysis Methods}

In spite of the time spent designing and gathering them, social networking data are only helpful insofar as they can be used, whether controlling for dependencies in data or providing the main source of phenomena to study (as in studies of influence). In this regard, analysis of social networks has somewhat lagged behind study design of social networks, but it is catching up rapidly, with many recent, exciting developments in the statistical investigation and inference of social networks. This section will begin with a review of common statistical treatments and models for controlling for social networks, then will shift to a discussion of studying influence in social networks, which will consist of challenges to the study of influence, as well as past and present models for the analysis of influence. To conclude this introduction and bring it full-circle, there will be a brief review of research in the contribution of social networks to academic engagement in grade-school students.

Actor dependencies. Social networks contain information about many types of actor dependencies (Snijders, 2011): 
Finding The Missing Links, 48

1) The most basic type of dependency is the reciprocal tie between two actors (Moreno, 1934) and amongst larger groups (Molm, Collett, \& Schaefer, 2007). This has been addressed by systems such as social exchange theory (in which reciprocal ties are thought to happen as a means of balancing an uneven exchange of resources; Emerson, 1976) and game theory (in which reciprocal ties arise as a cooperative gesture in a scenario where everyone stands to gain by helping one another; Molm, Peterson, \& Takahashi, 1999).

2) Homophily, in which similar actors will tend to form relationships with one another (Lazarsfeld \& Merton, 1954) and maintain them (Noel \& Nyhan, 2011). As is about to be discussed, this is just the first of many ways in which reciprocal ties are certainly not formed at random. Homophily will be discussed in more detail in the section on challenges to studying influence in social networks.

3) Transitivity of ties, in which actors will tend to form relationships with those they are indirectly connected with (Rapoport, 1953a; b). This tendency can lead to greater connectivity within local networks, giving rise to a large, interconnected group. As such, transitivity has also been referred to as clustering (Watts, 1999).

4) Differences in degree, with some actors having many connections and others having few, studied via centrality measures (Freeman, 1979). These differences can lead to densely connected actors having a disproportionately higher likelihood of forming new connections, leading to what has been 
Finding The Missing Links, 49

termed the rich-get-richer phenomenon (Price, 1976). This may exacerbate the variability in actor degrees, which may intensify core-periphery structures (Borgatti \& Everett, 1999) or lead to other types of network structuring.

5) Finally, directed networks often demonstrate hierarchies, in which the ordering of the out-degrees and/or in-degrees can change as we move from the center to the periphery of the graph (Lin, 1999). Such hierarchies may exist at the local or global level.

Thus, social networks indicate several ways in which actors are not randomly distributed throughout their social environments. In some cases, actor traits can determine network placement (Estell, Farmer, \& Cairns, 2007; Kossinets \& Watts, 2006). But, in turn, actor characteristics can often follow placement in social networks (Burkhardt \& Brass, 1990). In addition, an actor's ties may both reflect and influence their own traits (Lewis, Gonzalez, \& Kaufman, 2012). Thus, even when research hypotheses are not explicitly concerned with network position, controlling for it may be necessary to arrive at a less cloudy picture of the phenomena under study. One method, bootstrapping, will be discussed below. For a review of other methods involved in this, interested readers should see Appendix H.

Due to the violations of the assumptions of normality and independence, even relatively simple inferential statistical procedures, such as $t$-tests and ANOVAs, are prone to yielding incorrect standard errors. Instead, usage of non-parametric procedures, such as bootstrapping, are recommended (Bhattacharyya \& Bickel, 2013; Snijders \& Borgatti, 1999). In this procedure, anywhere between tens to thousands of "null" networks are 
Finding The Missing Links, 50

generated by randomly sampling from the observed network with replacement. Then, for each null network, some value of interest is calculated. These values will form a distribution of null values which the value from observed network can be compared against. Then, the $p$-value can be thought of as the percentile ranking of the value from the observed network amidst the distribution of null values.

Modeling influence. Actors' behavior can be influenced by both their position in the network and by their fellow group members. Authors of both discussion essays (Doreian \& Stokman, 1997; Emirbayer \& Goodwin, 1994) and thorough network studies (Padgett \& Ansell, 1993) have repeatedly argued that to deeply understand social action and social structure, researchers must study the dynamics of individual outcomes within networks. One fundamental dynamic is that people who are closely tied together tend to be similar in many behaviors and attitudes, a phenomenon referred to as network autocorrelation in the spatial statistics literature (Doreian, 1989). Explaining this network autocorrelation is necessary for measuring the relationship between actors' behaviors and attitudes. Unfortunately, this has proven to be a formidable task. The classic explanation has been of influence mechanisms, such as contagion (Christakis \& Fowler, 2013) and diffusion of innovations (Valente, 1996), while other researchers have proposed selection mechanisms, namely homophily, to explain similarities in connected actors (Lazarsfeld \& Merton, 1954; McPherson, Smith-Lovin \& Cook, 2001). Finally, context effects might also play a role (Feld, 1981; 1982). Each of these will be briefly reviewed in-turn.

Influence mechanisms. The notion that some idea, behavior, or trait can spread through a social network has existed since some of the earliest social networking studies 
(e.g., Moreno's study of runaways from an all-girls boarding school, in which girls whose social contacts had fled were often at a higher risk of fleeing themselves; 1934).

Christakis and Fowler's investigations into the Framingham Heart Study, an epidemiological cohort survey of 12,067 individuals over 32 years, have provided many of the findings in the field of social influences (for a review, see Christakis \& Fowler, 2013). They employ a metaphor of ideas as a form of "social contagion", spreading from person-to-person like a bacterium or virus. In their research, Christakis and Fowler have uncovered two particularly intriguing general findings about social influence and contagion.

First, actors generally show "three degrees of influence" ${ }^{1}$, in which actors and the friends of their friends' friends show a greater correspondence on many traits than would be expected by chance. This has been found in Christakis and Fowler's work (2008; Fowler \& Christakis, 2010) as well as that of other authors, such as research in word of mouth advertising (Brown \& Reingen, 1987), knowledge contained by inventors (Singh, 2005), and happiness in large twitter datasets (Bliss, Kloumann, Harris, Danforth, \& Dodds, 2012). The number was not always three, Christakis and Fowler found it to be two in a study of divorce (McDermot, Fowler, \& Christakis, 2013), and four in a study of sleeping disturbances and drug use (Mednick, Christakis, \& Fowler, 2010). Furthermore, the pattern was not universal, as some traits showed no measurable spread, such as sexual

\footnotetext{
${ }^{1}$ Christakis and Fowler themselves acknowledge that these should be more properly called "three degrees of clustering" than "three degrees of influence", as the selection and influence contributions have not yet been teased apart in this formulation. Nonetheless, the phrase was intended to be "evocative" and meant to illuminate that "there is evidence that diverse phenomena spread beyond one degree" and that "there is evidence that the association fades within a few degrees in what seems like a systematic way across phenomena and datasets." (p. 8, Christakis, \& Fowler, 2013)
} 
Finding The Missing Links, 52

orientation (Mednick et al., 2010) and health screening behaviors (Keating, O’Malley, Murabito, Smith, \& Christakis, 2011). However, three appears to be the modal number for the moment.

Christakis and Fowler suggested that this number might be due to a decay in the influence, or a decline in the size of meaningful and detectable effects, the further out analyses go and to the nature of geodesic distance (the number of nodes in the shortest walk for any given pair of actors). Two indirectly connected actors are dependent upon the ties between them to reach one another. However, over the course of life, ties can break: people die or move away, friends grow apart, couples leave one another, etc. Thus, these broken ties also break any indirect ties that they were bridging. As such, the stability of ties at four and more degrees of separation may be too low to sustain an influence effect.

Christakis and Fowler's second interesting finding was that whereas they expected for the decay in influence to be exponential (for example, if Jill has a $30 \%$ chance of influencing Melissa, and Melissa has a 30\% chance of influencing Robin, then Jill ought to have a 9\% chance of influencing Robin), they actually found the decay to be slower than this. Of this, Christakis and Fowler extend the contagion metaphor to speculate that some people might act as "carriers" who may transmit some sort of trait without exhibiting it themselves. As of yet, this notion has not been explored, nor has a “carrier" been operationalized. Another possibility, not considered by Christakis and Fowler, is that some actors possess certain properties that may moderate how influential they are. Some of these properties have been indicated by other research. 
Finding The Missing Links, 53

The diffusion of innovations is a model that was introduced by Ryan and Gross (1943) in which some small quantity of members adopt an innovation, then their contacts adopt the innovation, and so forth until all (or most) members adopt the new idea. Under this model, those who adopt the idea earliest are called "innovators", then comes the "early majority" (those who adopt the idea before half of the population has, but not at first), then comes the "late majority", then finally there are the "laggards" (those who adopt an idea after the vast majority of the population has) and the "non-adopters" (those who never adopt). Valente has extended this idea to personal networks (1996), in which he argues that some forms of diffusion take a sort of bandwagon effect, causing people to adopt the idea once enough of their contacts have adopted it. Borrowing the language of Ryan and Gross, Valente posits that some people will be innovators, in that they will adopt an idea before few, if any, of their contacts have adopted it, while others will be part of an early majority (who adopt an idea before reaching the average threshold), late majority, or laggards (who will wait until nearly everyone in their personal network has adopted the idea before they join in) and non-adapters. Research in this field has indicated that certain network positions confer advantages to actors in spreading a message across a network.

Burt $(1980 ; 1987)$ argues that structural equivalence, or the similarity of who two given actors are connected and not connected with, promotes adoption and influence. He compares two actors with an identical set of connections to two siblings of similar ages. Given their similarities, each sibling will need something extraordinary to make them stand out, and they may be eager to be an innovator. The other sibling would then be 
Finding The Missing Links, 54

expected to adopt the new idea quickly to keep up. Other researchers have found that those who adopt ideas quickly and influence others to adopt tend to be more highly central, have more densely connected networks, and have a higher proportion of reciprocal connections (Rice, 1993; Valente, 1995). One property in particular merits a more in-depth look.

Granovetter is famous for writing on the "strength of weak ties" (1973). In his paper, he distinguished between two senses when discussing the strength of ties. In a relational sense, the strength of the tie is a channel of knowledge. Thus, weak ties are those between people who interact infrequently, have little investment in the relationship, and are less easily influenced by one another. On the other hand, in a structural sense, the strength of the tie refers to the ability of the tie to link otherwise distant nodes. He notes that, often, relationally weak ties prove to be structurally strong ones. Thus, under this theory, influential people will not be those with many strong ties in a closely knit network, as this will essentially create a sort of "idea inbreeding": everyone is familiar with the same information, and so ideas tend to be redundant. Instead, those loosely connected nodes who are more likely to adopt an actor's idea due to its novelty, are also likely to spread that idea to a number of other actors who will similarly adopt the idea due to its unfamiliarity. Support for this notion has been found in a wide variety of research, such as studies of business information (Levin \& Cross, 2004), job searching (Montgomery, 1992), creative ideas (Baer, 2010), technical advice (Constant, Sproull, \& Kiesler, 1996), criminal behaviors (Patacchini \& Zenou, 2008), political lobbying 
Finding The Missing Links, 55

(Carpenter, Esterling, \& Lazer, 1998), and even amongst non-human primates

(Maryanski, 1987).

One possible exception to this theory may be in complex contagions. Centola and Macy (2007) write that the contagion metaphor starts to break down when considering some of the differences between communicable diseases and ideas. For example, most infectious diseases are caused by a single class of microbes and, as such, only require a minimum of a single source of contact. However, many ideas are complex, and may require multiple sources of contact to transmit the full idea (it should be noted that some diseases work like this as well. For example, fulminant and subfulminant viral hepatitis involves infections of multiple hepatitis viruses; Wu et al., 1994). Their brief review of the literature found support for this assertion, although the authors did not define what a complex idea was, which may lead to some tricky circular reasoning: is a complex idea any idea which simply does not obey the strength of weak-ties theory?

Selection mechanisms. Could all similarities between pairs of connected actors be due to socialization effects? Certainly not all, as why would the actors establish a tie in the first place, if they are so dissimilar? It is well-known that people prefer to be in the company of similar others, a notion called homophily (Lazarsfeld \& Merton, 1954). Under this explanation, actors can be thought of as static and the network as dynamic (Steglich et al., 2010). Homophily is a well-documented phenomenon, having been informally recognized since antiquity (Aristotle, 1934; Plato, 1968) and empirically recognized since the earliest social networking studies, in which researchers found that children were more likely to form friendships and play with one another when they were 
Finding The Missing Links, 56

more similar on demographic characteristics (e.g., Bott, 1928; Hubbard, 1929; Wellman, 1926). It has been documented in a wide variety of relationships, from the closest ties of marriage (Kalmijn, 1998) and friendship (Verbrugge, 1977; 1983) to more casual relationships such as preferred coworkers (Ibarra, 1992; 1995), people that we "know about" (Hampton \& Wellman, 2001), or people that we are willing to appear with in a public place (Mayhew, McPherson, Rotolo, \& Smith-Lovin, 1995).

In their seminal paper on the topic, Lazarsfeld, \& Merton (1954) described two types of homophily: status homophily, in which similarity is based on formal, informal, and ascribed status; and value homophily, in which similarity is based on values, attitudes, and beliefs. In status homophily, major demographic traits such as age, sex, race, ethnicity, religion, education, occupation, etc. that are used to stratify society are a basis for relationships. Meanwhile, in value homophily, more personal characteristics such as emotions and thoughts serve as another basis for relationships. Often, value homophily proves to be derivative of status homophily (McPherson et al., 2001).

Past research has indicated at least four basic causes of homophily. The first and possibly most straightforward is geographic space, a finding best illustrated by the classic community studies (for a review, see Campbell, 1990), and impacted by such seemingly trivial factors as the arrangement of streets (Hampton \& Wellman, 2000; Sudman, 1988), legislative seating (Calderia \& Patterson, 1987), and dorm halls (Kun, Guangyi, Deshun, Jun, \& Jinrong, 2014). To Zipf (1949), the finding is a simple case of effort: connections will require more energy for those who are far away than for those who are close. Recently, due in no small part to the rise of the internet and social media, this relationship 
Finding The Missing Links, 57

is attenuating in some aspects, as can be seen in social networking studies of health behaviors for example (Christakis \& Fowler, 2007; 2008; Rosenquist, Murabito, Fowler, \& Christakis, 2010), however even some online social networks display geographic clustering (Scellato, Mascolo, Musolesi, \& Latora, 2010).

Family ties are another common source of homophily (Louch, 2000), although the picture is somewhat nuanced. Research finds wide support that most actor characteristics in kin ties tend to be similar in terms of status homophily, however strong, homophilous, ties on one characteristic can act to induce heterophily on other characteristics (McPherson et al., 2001).

A third common cause of homophily in social networks is organizational foci, such as school, work, or voluntary groups (such as charities or recreational clubs). In Louch's study (2000), the vast majority of non-kin ties among actors were contained within these sorts of centers.

Finally, a fourth cause of homophily is in who actors choose not to remain with. Homophily is generally thought of as the formation of ties between people who are similar, however it has also been found to apply to the dissolution of bonds (Noel \& Nyhan, 2011). Amongst children, same-sex and same-race ties have been found to be more stable than cross-sex and cross-race ones (Hallinan \& Williams, 1989; Tuma \& Hallinan, 1979), and bankers' ties with others tend to last longest when the partner is a fellow banker of a similar age (Burt, 2000), while members of recreational clubs who are more dissimilar to that club's niche are more likely to leave than those close to it 
Finding The Missing Links, 58

(Popielarz \& McPherson, 1995). Thus, homophily can result in actors resembling one another due to the fact that actors tend to seek out similar others and stay with them.

Context effects. Similar to third-variable causation, context effects refer to those unmeasured variables that can cause similar changes in actors and connected nodes, thereby giving off the appearance of either contagion or homophily. Context effects often appear in two forms, as environmental context and structural context.

Environmental context refers to properties of the environment which can influence actors' behavior, homophily, or contagion. The possibilities are virtually limitless and will depend on the study situation. One particularly prominent property concern large-scale geographical features and standard demographics (Sampson, Morenoff, \& Gannon-Rowley, 2002), which can effect risky behaviors (Adimora \& Schoenbach, 2005), access to others (McPherson et al., 2001), and communication patterns (Christakis \& Fowler, 2013). The internet has attenuated these effects somewhat, however it has its own properties, such as anonymity (Bargh \& McKenna, 2004). One's culture has also been shown to interact with social networks to constrain the flow of information across computer-mediated communication groups (Cho \& Lee, 2008).

As such, without knowledge of what to look for or a bit of luck in measurement items these effects cannot generally be detected. However, as noted by Snijders, "[m]any network studies are about only one single network and in that sense are $N=1$ studies." (2011, p. 135). As such, it is important to recognize that these social network studies are inadequate for generalizing to external populations anyway, not without several 
Finding The Missing Links, 59

replications in other samples and use of multilevel modeling methods to control for context effects.

Structural context refers to the organization of the social network and where actors fall, both in relation to the overall network and each other. Such positioning can effect outcome variables. For example, student academic performance has been shown to be positively related to their Katz-Bonacich centrality (Calvó-Armengol, Patacchini, \& Zenou, 2009), a measure whose stability is highly dependent on the network properties under study (Costenbader \& Valente, 2003). Relationships between adolescent substance abuse and network properties and position have also been found (Ennett et al., 2006). Finally, over the course of a series of experimental and observational studies, Visser and Mirabile have found that attitude strength is related to homogeneity of one's social network (2004). Specifically, when a person is in a social network of people who hold an opinion similarly to them, they experience less ambivalence and are less receptive to arguments against their position than if there is great heterogeneity in the network.

The above examples highlight some of the ways in which network position and structural variables can influence individual outcomes. However, unlike with the environmental context effects, these structural effects can be measured and controlled for without additional design in the study. Nonetheless, in the cases of whole social network measures, the results will be most generalizable to other networks with similar properties. Thus, studies of multiple social networks are still necessary to obtain enough variation in social network properties to be able to generalize to a wide variety of social network structures. 
Finding The Missing Links, 60

Procedures for modeling influence. In previous models, network dependence and structure was often a nuisance, and sometimes a source of predictive variables. As such, they were not often viewed as formative factors in and of themselves, rather worked in concert with other independent variables to influence outcomes. However, other social networks researchers put the social network front and center in proposing it as a primary (or even the primary) cause of actor behavior. Essentially, any parent who has ever worried about their children's friends being "bad influences" or managers who hoped that good employees would influence others have adopted this view. Analyzing this assumption has turned out to be a very troublesome matter, unfortunately. A description of some of the challenges for analyzing influence in social networks will be presented, followed by a brief review of four of the major procedures which do anyway.

In order to statistically disentangle the effects of homophily and influence, Pearson and colleagues write that there are three key issues to overcome (2010): first, as the actors of the social network are not independent, statistical techniques which assume independence are not appropriate and can lead to under- or over-inflated standard errors, increasing the probability of type 1 or type 2 errors (Kenny \& Judd, 1986).

Second, alternative mechanisms of similarity must be controlled for. For example, in the phenomenon of network closure (Feld \& Elmore, 1982), imagine a dyad of actors $\mathrm{A}$ and $\mathrm{B}$, and a third actor $\mathrm{C}$, with whom A shares a connection but B does not. Due to her connection with A, B can be expected to come into contact with $\mathrm{C}$, and should eventually form a direct relationship with them. This phenomenon is not universal, as 
Finding The Missing Links, 61

some actors otherwise seek to maintain a structural hole, a decision with some psychological antecedents (Kalish \& Robins, 2006).

Third, incomplete observations of social networks while the underlying formation processes operate in continuous time. For example, if two actors, A and B, have suddenly become similar to one another and formed a connection in between collection points, two orderings of processes need to be considered: did A and B form a relationship, then socialize one another to become more similar (influence)? Or, did A and B become similar to one another due to external processes and then form a relationship because they had so much in common (homophily)? Other, more complicated, processes must be considered as well, such as the presence of other actors. The following statistical procedures have differing levels of success in overcoming these challenges.

Contingency tables. In this approach, the social network is divided into dyads of mutually nominated connections. These dyads are, in turn, cross-tabulated by whether the actors' connection remains stable from the first to the second measurement, and whether the actors' behavior is the same on some binary variable (ex. "drinks/does not drink alcohol"). The probability of behavioral similarity is then assessed for two subsets of the data: friends who stayed together (which assesses influence) and friends who did not (which assesses selection). These probabilities can be compared to the observed data to derive hypothesis tests.

This method tends to appear in older social networking studies, such as Kandel's study of marijuana use amongst adolescents in New York (1978), Billy and Urdy's study of sexual behavior in adolescents (1985), and Fisher and Bauman's study of adolescent 
Finding The Missing Links, 62

smoking and alcohol consumption (1988). The assumption of dyad independence is typically acknowledged as an unavoidable weakness, while the other two objections go completely unaddressed. In particular, the division of the social network into dyads eliminates any possible analysis of structures comprised of three or more actors, such as network closure.

Aggregated personal networks. Studies that employ this strategy generally follow a two-step procedure (Ennett \& Bauman, 1994; Kirke, 2004; Pearson \& Michell, 2000; Pearson \& West, 2003): first, network data are condensed into summary statistics at the level of the actor, representing the actor's network position and various properties of her personal network. Most often, network position is categorized using the negopy software (Richards, 1995), which places actors into four basic sociometric positions: group member, peripheral, liaison, and isolate. Second, these abbreviated data are analyzed with assumptions of independence.

To tease out selection and influence effects, a contingency table-like approach is used. In this case, selection is presumed when a target actor who exhibits a certain behavior is not part of a group exhibiting that same behavior at time 1, but is part of that group at time 2. Meanwhile, influence is presumed when an actor who does not exhibit some target behavior is part of a group of individuals who do exhibit it at time 1, but then exhibits it at time 2 . The cases of isolated actors who both join a group of individuals with a certain behavior and adopt that behavior between time 1 and time 2 are disregarded, due to the ambiguity surrounding ordering, though sometimes they are interpreted as being influenced by a group they just joined (Pearson \& West, 2003). 
Finding The Missing Links, 63

Given its similarities to the contingency-table approach, many of the same limitations apply. On the one hand, this approach does have the advantage of being able to control for local network structures, but this approach also suffers from being unable to model relationships with specific actors (due to the aggregation), whose traits and/or network positions may confer privileges in influencing others.

Actor-partner interdependence model. This model enjoys wide use today, building on the ease with which complex models can be analyzed owing to contemporary statistics software and fast computing (Cacioppo, Hawkley, \& Thisted, 2010; Christakis \& Fowler, 2013; Markowitz, Bellair, Liska, \& Liu, 2001; Steinfield, Ellison, \& Lampe, 2008). In this model, the value of an actor's partners on some variable of interest is averaged together for two waves of data collection (at least). Then, a structural equation model is specified in which previous wave actor value and previous wave average partner value predicts current wave actor value and current wave average partner value. Thus, selection effects can be assessed by examining the coefficient of the path from previous wave actor value to current wave average partner value (while holding previous wave average partner value constant) and influence effects can be assessed by examining the path from previous wave average partner value and current wave actor value (while holding previous wave actor value constant). See figure 1 for an illustration.

Compared to the previous designs, this one allows for the analysis of a wider range of network positions and actor traits, and the separation of selection and influence is somewhat more statistically rigorous and encompasses the entire sample. Nonetheless, personal networks are still aggregated, thus limiting exploration of alternative generating 
Figure 1. Actor-partner interdependence model

Time 1

Time 2

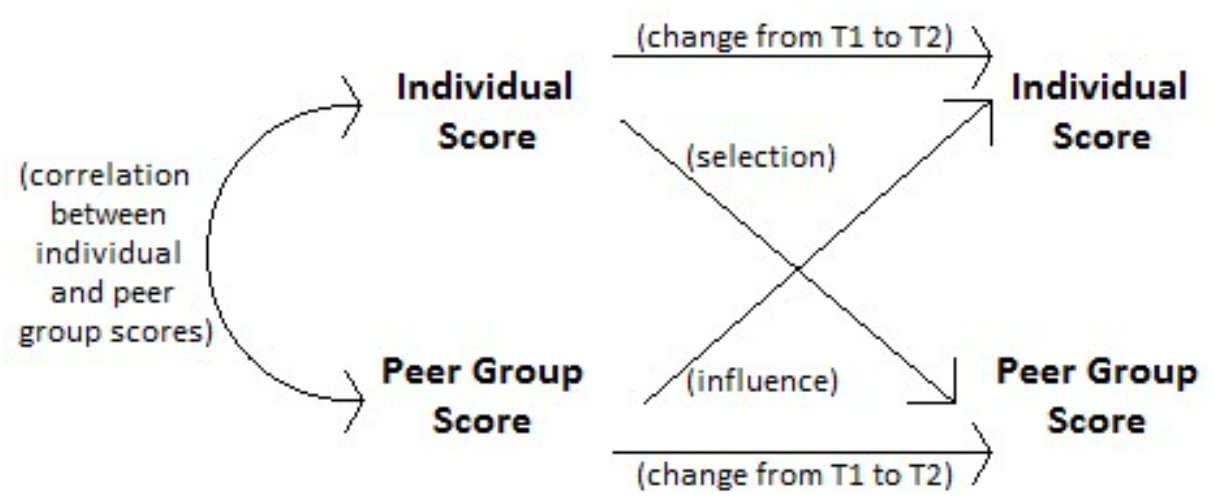

Statements in parentheses indicate what is being statistically controlled for

mechanisms. In addition, the extent to which selection and influence are truly, and completely accounted for, without confounding, is a matter of debate. Some argue that homophily and influence can never be disentangled from one another (Shalizi \& Thomas, 2011), a sentiment reflected by a study using the similar cross-lagged panel design to find evidence of contagion in headaches, acne, and height amongst adolescents (Cohen-Cole \& Fletcher, 2008). Christakis and Fowler, however, note that the authors of that study used a very liberal significance level for two of the traits $(\alpha=.10)$, and that the effect sizes were extremely weak (2013). Nonetheless, the presence of challenges to a method do not automatically condemn the use of that method. Christakis and Fowler go on to argue that researchers must make the most of what they can, even if the methods are imperfect, otherwise we will all be carrying out double-blind, placebo-controlled studies on the effectiveness of parachutes (see Smith \& Pell, 2003).

Pearson and colleagues have developed a new model called "stochastic actorbased modeling" which they argue counters all of the above limitations, however its 
Finding The Missing Links, 65

freshness has posed some concerns about its validity and it will not be employed in this thesis. Interested readers may examine an overview of it, and these concerns, in Appendix I.

Social networks and academic engagement. The prominence with which the social context plays in the lives of early adolescents has long been recognized by educational researchers (Eccles, Lord, \& Midgley, 1991; Eccles et al., 1993). As discussed in the section on friendship, children begin to form relationships with other children very early in life, and the significance of their friends and peers only increases with age, especially so during the middle school years. Discussion of middle school reform commonly features a great deal of attention on students' need to belong and their desire for a sense of connectedness, as well as the functions provided by friends and peers (Juvonen, 2007; Lipsitz, Jackson, \& Austin, 1997). This emphasis is not surprising, given the effect that peers can have on the academic engagement of one another. Research has consistently indicated that students' academic engagement is associated with their peers' (Chen, 2005; Liem \& Martin, 2011; Nelson \& DeBacker, 2008; Ryan, Stiller, \& Lynch, 1994; Simons-Morton \& Chen, 2009; You, 2011).

However, in these studies, students were asked to report on their peers' level of engagement, as well as their own. Thus, the relationship was between student engagement and perceived peer engagement. Past research has indicated that students tend to overestimate how similar they are to their friends (Bauman \& Ennett, 1996; Berndt \& Keefe, 1995; Davies \& Kandel, 1981), which can inflate correlations between students' and friends' behavior. These concerns are often justified with the argument that 
Finding The Missing Links, 66

what students think about their friends is more influential than what they are. However, perceived and real reports of academic engagement tap into different processes of peer influence, and there has yet to be a clear distinction of what those are (Ryan, 2000).

Of studies that directly examine the academic engagement of students and peers themselves, the pool of research is much smaller. Kindermann (1993) performed one of the earliest such studies, measuring the academic engagement of students in two fourthand two fifth-grade classrooms in the fall and spring of one school year, using sociocognitive mapping methods and binomial tests to measure the classrooms' peer networks. Peer group engagement was averaged relative to each actor and analyses were performed using multiple regression. He found evidence of selection and influence for the relationship between peer group average engagement in fall and changes in individual engagement from fall to spring. Interestingly, despite a fairly high rate of member turnover in groups (about 50\%), peer group average engagement stayed fairly consistent between time points $(\mathrm{r}=.80)$. However, he found more variability in student-to-group engagement differences, and suggested that engagement may become a less important organizing force of children's peer groups over time (though, with the consistency, it is apparently still at least somewhat important). Overall, influence processes showed a stronger effect than selection processes.

Kindermann (2007) performed a second study on the entire sixth-grade of a middle school, using the same methods as his previous study. As before, he examined the relationship between peer academic engagement in the fall and changes in individual academic engagement from fall to spring. As before, he found significant effects for peer 
selection and influence (this time, selection proved stronger) on changes in individual engagement across the school year. Overall, peer influence accounted for $2 \%$ of changes in student engagement, but this varied with the amount of change in influence: for students whose fall engagement was above the median, peer influence accounted for nothing. For those below, peer influence accounted for 3\%, while for the 41 students whose engagement changed by at least one standard deviation, peer influence accounted for $13 \%$. In his previous study, Kindermann examined selection effects by predicting spring peer engagement with fall individual engagement, whereas this one examined selection by using peer group properties (such as group size, group stability, and percent of same-sex peers).

Ryan (2001) measured self-reported academic engagement and the social network of a seventh-grade student body in a middle school via reciprocal friendship nominations. Reciprocal ties were then organized into non-overlapping friendship groups to permit use of hierarchical linear modeling (HLM) for analyses. Using the intraclass correlation, she estimated peer selection effects to account for about $14.3 \%$ of the variance in individual student engagement in fall, and $14.0 \%$ of the variance in spring. Between-group models indicated that peer context (interpreted as influence) accounted for $48 \%$ of the variance in student engagement.

Molloy and colleagues (2011) performed a comparison of social network research methods in examining each method's ability to predict changes in individual engagement from fall to spring, using fall peer engagement. The researchers compared social networks based on reciprocal friendship nominations, socio-cognitive mapping with 
Finding The Missing Links, 68

binomial tests (which they termed interaction dyads), and socio-cognitive mapping with principle components analysis (which they termed social groups) on a fifth- and seventhgrade cohort and found them all significantly related to changes in engagement when examined individually. When combined into a single model, none of the three types of relationships were significantly related to spring engagement for fifth graders, while only friends were significant for seventh graders.

Finally, two studies of friendship networks using stochastic actor-based modeling found evidence of friend engagement influencing changes in student engagement across the school year, as well as evidence of selection and network effects (Berk, Kerr, \& Stattin, 2006; Shin \& Ryan, 2014). Both studies generally indicated that selection processes exerted a stronger effect than influence.

Thus, of the six studies found that directly measured peer networks and peers' engagement, five examined selection and influence as separate processes. All studies agreed that peers' academic engagement in the fall was related to changes in individual students' academic engagement across the year, but what these were primarily due to varied. Two studies generally indicated that influence processes showed stronger effects than selection, while three studies found the opposite (although significant selection and influence effects were found in all five studies).

Part of this disagreement in findings may be due to the fact that studies went about obtaining those findings in different ways. Kindermann measured participant observed interaction partners and used a binomial test to establish a link between peers who received more nominations than they should have by chance. Analyses were 
Finding The Missing Links, 69

performed via a cross-lagged panel design. Ryan, on the other hand, measured friendships and then eliminated ties according to rules with arbitrary cutpoints (such as that at least $50 \%$ of a group member's direct ties had to be within the peer group) to perform analyses with HLM. Berk and colleagues and Shin and Ryan also measured friendship networks, but used stochastic actor-based modeling. There was no study using the socio-cognitive mapping method that included either Cairns and colleagues's criteria for dividing individuals into groups (1991) or any sort of factor analytic method.

Social network context. The context of a social network can be measured by the calculation of metrics. Several of these metrics have shown a relationship with student outcomes, sometimes even directly affecting academic engagement.

One of the most common and basic metrics is density, which is simply calculated by dividing the number of present ties by the number of potential ties. For instance, in a network with $50 \%$ density, half of the potential ties are present. This can be calculated for both the network as a whole, and for individuals. For individuals, this measure has shown a negative relationship with how often students interact with other members of their network (Fournet \& Barrat, 2014) and how many others they nominate in network studies (Burk, Kerr, \& Stattin, 2008; Shin, 2014; Steglich, Snijders, \& Pearson, 2010).

Another common metric is centrality, which is based on a notion that some people are prominent or influential in their networks. Academic outcomes have been shown to vary depending on the centrality of a student in their social network (Estell, Cairns, Farmer, \& Farmer, 2002; Farmer \& Farmer, 1996; Farmer \& Rodkin, 1996). Unfortunately, the devil is in the details, because operationalizing "prominence" and 
Finding The Missing Links, 70

"influence" have proven tricky. Costenbader and Valente (2003), for example, identified nine common measures of centrality, some based on number of connections, some based on paths, and some based on similarity of ties with others.

Grade school social networks often show a great deal of modularity, or a structure comprised of a series of communities in which members are densely interconnected, while being only sparsely connected with non-members (Badge, Saunders, \& Cann, 2012; Girvan \& Newman, 2002). As will be discussed in "Methods Comparison" section of the "Network Identification Methods" unit, the manner in which the social network data are collected and analyzed can greatly contribute to the extent of modularity.

Average path length is an individual metric representing how many actors an individual would need to pass through to reach a particular other (Badge et al., 2012). Such information is useful in studies of the spread of gossip (Lind, da Silva, Andrade Jr., \& Herrmann, 2007), for example. Although, as Borgatti (2005) warns, information does not always follow the shortest possible path, or even a single path.

A common goal in social network analysis is to determine the effect that the social network has on individual changes in the value of some trait, such as antisocial behavior or academic engagement. Several metrics have been employed in the pursuit of this goal.

In the analysis of a social network, calculating the clustering of a trait can be one of the earliest methods of determining if the structure and composition of the social network are related to the distribution of an idea or behavior. McFarland, Moody, Diehl, Smith, and Thomas (2014) argued that clustering in adolescent social networks is 
Finding The Missing Links, 71

virtually inevitable, as "population size, differentiation, composition, and climate, all interrelate to amplify or impede general mechanisms of network formation" (emphasis theirs). Eventually, they argue, "the salience of all tie-formation mechanisms is amplified so as to create segregated and hierarchically clustered networks of relations" (p. 1094). Clustering has been found in studies of delinquency (Pearson, Steglich, \& Snijders, 2006) and academic engagement (Shin, 2014). Three processes are often involved in clustering: selection, influence, and context.

Assortativity, or a tendency to associate with similar others, has been well documented in grade schools, turning up in studies of gender and ethnic preferences (Fournet \& Barrat, 2014; González, Herrmann, Kertész, \& Vicsek, 2007), antisocial and delinquent behavior (Burk et al., 2008; Estell et al., 2002; Pearson et al., 2006), social interactions (Fournet \& Barrat, 2014), and academic engagement (Kindermann, 1993; McFarland et al., 2014; Shin, 2014). This effect is not thought to contribute to changes in individuals, rather it is often viewed as a confound in social networks influence research: if an individual is found to be more similar to their peers from at time 2 (relative to time 1), is it because the individual and their peers have influenced one another, or simply because the individual has chosen like-minded peers? Assortativity is simply a measure of how similar one is to their peers at a particular point in time, and so it cannot easily answer this question by itself. A more thorough discussion of this will be presented in the "Modeling Influence in Social Networks: Challenges" section in the "Analysis of Social Networks" unit. 
Finding The Missing Links, 72

A somewhat related notion is transitivity, in which partners of partners will often become partners themselves. For example, if $\mathrm{A}$ is friends with $\mathrm{B}$ and $\mathrm{C}$, and $\mathrm{B}$ and $\mathrm{C}$ do not currently know one another, it is generally more or less a matter of time before they will become friends as well (this is also known as "filling a structural hole"). Transitivity has been well-documented in longitudinal social networking studies of grade school students (Burk et al., 2008; McFarland et al., 2014; Pearson et al., 2006; Shin, 2014). As such, it is a cause of network change that is related neither to selection or influence (even relatively dissimilar actors will tend to fill in structural holes; Flynn, Reagans, \& Guillory, 2010), thus indicating it is a context effect (a phenomenon of the network environment). Transitivity has been recommended as a control variable in studies of the spread of ideas and behaviors in social networks (Steglich et al., 2010). 
Finding The Missing Links, 73

\section{CHAPTER 3: The Current Study}

In this study, I comprehensively compared the two major collection methods of social network information used in school settings by comparing student self-report methods with participant observations. In self-report methods, students listed those peer affiliates whom they regard to be members of their own groups. In participant observation methods, students were asked to report about all peer groups and their members they see at school. In addition, in this study I compared the four most commonly encountered network identification methods in the literature: reciprocality of self-reported partnerships, probability-based identification of group members, factoranalysis methods to define groups, and decision-rule strategies.

The sample for the current study is the entire population of $3666^{\text {th }}$ graders in the sole middle school of a small town. This sample makes it possible to examine the completeness of the social networks that are identified by the different methods. In terms of collection methods, it is generally expected that participant observations will be the more exhaustive network measurement method and will lead to identifying more complete social networks by covering more possible members of networks than would be obtained by self-reports. With regard to identification methods, networks identified by probabilities are generally expected to show the greatest proportion of students captured, and to be least biased by students' academic engagement levels. Finally, several exploratory analyses will be performed to better understand the differences between the four networks. 
Finding The Missing Links, 74

\section{Network Measurement}

Four hypotheses will be examined to test whether participant observation methods allow more inclusive (complete) network measurements than self-reports:

Hypothesis 1: The percent of the student body captured (i.e., represented in the data, regardless of significance of ties) by participant observations will be significantly higher than the percent of the student body captured by self-reports.

The participant observation method has several advantages for capturing potential network members, compared to the self-report method. First, network information about students not present for a data collection can be captured by those observers who are present. Second, tie information comes from several students who may not necessarily know the individuals in question personally, but have observed them. Thus, one informant who knows of a certain tie but neglects to mention it may be compensated for by the huge set of additional observers. In self-reports, an absent student can only be captured (e.g., as a friend) if he or she is mentioned by anyone who considers that student a friend, hence there is a much smaller base of informants with which to capture potential network members. As such, a significantly higher percentage of the student body should be nominated at least once by participant observations than by self-reports.

Hypothesis 2: The percent of boys captured by participant observations will be significantly higher than the percent of boys captured by self-reports. 
Finding The Missing Links, 75

This expectation is based on a previous finding that boys were slightly less likely to participate in a study on friendships and academic development (Kindermann, 2007). There are several other reasons to expect participant observations to capture boys to a greater extent than self-reports. As previously discussed, boys and girls show striking differences in their peer networks by middle school: boys tend to associate in large, loosely connected play groups, while girls tend to focus on a few, close dyadic friendships (Thorne, 1992). As such, more boys should be nominated via participant observations than by self-reports, for several reasons: first, boys who do not feel close enough to certain common play partners may not think of them as friends, and so they may be reluctant to nominate them as such, resulting in partners being under-reported when eliciting network information via self-reporting (partial support for this assertion comes from Shrum, Cheek, and MacD, who found that children from grades 3 to 12 nominated friends that they "spent the most time with" less consistently than "best friends" [1988], although a breakdown by gender was not provided). Second, the sheer amount of partners may make boys more prone to forgetting friends than girls. Third, due to the frequency of interaction with boys in their groups, informants should be plenty capable of identifying their frequent interaction partners.

Hypothesis 3: The percent of girls captured by participant observations will not significantly differ from the percent of girls captured by self-reports.

No significant differences are expected for girls. As their friendship networks tend to be smaller and closer than boys', girls are not expected to accidentally neglect as many of 
their friends in self-reports, and participants should be able to report on girls' dyads, which are viewed in public often enough to be readily identifiable.

For a student to go completely uncaptured by one of the measurement methodologies, two events must happen: First, the student would not provide a report of his or her friends or social network partners (for example, due to lacking permission, missing the day of data collection, or refusing to participate either actively or passively). Second, the student would not be nominated as a friend or network member by any other student. As I will argue, disaffected students should meet the first criterion fairly evenly for both measurement methods, but should meet the second criterion more severely for selfreported friendships than for participant observations.

Hypothesis 4: Academic engagement should be a significantly weaker predictor of being covered for the participant observation method, compared to the self-report method.

As argued by Skinner, Kindermann, and Furrer (2008), academically disaffected students are less likely than academically engaged youth to participate in classroom activities, which should include academic research. As such, the most at-risk youth are theoretically predisposed to not participate in research, thus under-representing themselves in the data. One possible indicator of this, that disaffected children may have removed themselves from social network data collection or refused to take part, was rejected by Kindermann (2007) because no significant difference was found in the academic engagement of children who provided social network reports compared to those who did not. However, 
Finding The Missing Links, 77

another possibility is that disaffected students may have completed social network reports but either have left out information about themselves, or indeed have no affiliates.

In addition, as disaffected students tend to have few friends and small networks (Kindermann, 2007), self-reported friendships may be an inappropriate method for capturing such youth, as the concerns over self-report are only magnified for students with few friends. Instead, participant observations may be more appropriate due to the larger pool of informants, which will help compensate for the smaller networks. Thus, self-report methods are expected to be biased against disaffected students, while participant observations are expected to be less so (if at all).

\section{Network Identification Methods}

Once data have been gathered, information needs to be aggregated to generate the ties that form a single network. For this study, networks will be generated via the four previously mentioned identification strategies (reciprocity for self-reported friendships, and probabilities, factor analyses, and decision rules for participant observations). In the next set of hypotheses, I expect that the network identified by probabilities will be most inclusive in proportion of students captured (i.e., students identified as having one or more partners) and least biased against disaffected students. Four hypotheses will be examined: 
Hypothesis 5: Across the networks identified using the four identification methods, the network identified by probabilities will capture the largest proportion of the student body.

Hypothesis 6: Across the networks identified using the four identification methods, the network identified by probabilities will show the weakest relationship between whether a student is male and the probability that that student is captured.

Hypothesis 7: All four networks will not significantly differ in the relationship between whether a student is female and the probability that that student is captured.

As boys typically associate in large, loosely connected networks, probabilities are expected to capture their ties more effectively than factor analyses and rule-based approaches (both of which require more densely connected networks). The smaller, more tight-knit networks of girls, however, should allow for a relatively even opportunity to be captured by any of the network identification methods.

Hypothesis 8: In networks identified via each of the four methods, the average engagement of students with no identified partners will be significantly lower than the average engagement of students with at least one identified partner.

The expected processes underlying hypothesis 8 is the same as the ones underlying hypothesis 4 , that academically disaffected students will be less likely to provide 
information about their own networks, and that their smaller social circles will render them less likely to be nominated as a friend or member of a peer group.

\section{Research Questions}

In addition to these hypotheses about network completeness and reliability, several exploratory analyses will be conducted to better understand the differences between the four identified networks. These analyses have two goals: The first is to show that network completeness makes a difference for all subsequent network characteristics that can be defined and relationships that can be examined. The second is to show the extent to which network analysis methods can add to these initial differences. The research questions (RQs) are as follows:

\section{RQ 1: How much overlap do networks demonstrate?}

RQ 1.a: How much agreement do identified networks show on existing ties? How much agreement do identified networks show on absent ties? How much agreement do identified networks show overall?

$R Q$ 1.b: Are there differences in the correlation?

$R Q$ 1.c: Are there differences in the interrater agreement?

Overlap is a frequently reported statistic in network comparison studies, as discussed in the "Comparison of Self-Reports with Participant Observations" section. I will follow suit by using the most commonly-found methods: percent of agreement in existing ties (given that network 1 reports a tie between $\mathrm{A}$ and $\mathrm{B}$, how likely is network 2 
to report the same tie?), percent of agreement in absent ties (given that network 1 reports no tie between A and B, how likely is network 2 to also report no tie?), percent of agreement overall (what percent of the time do networks agree with one another?), correlation, and interrater agreement.

RQ 2: How do networks compare in whole-network metrics?

RQ 2.a: Are there differences in the density?

$R Q$ 2.b: Are there differences in the modularity?

$R Q$ 2.c: Are there differences in the level of transitivity?

RQ 2.d: Are there differences in the clustering of academic engagement?

Whole-network metrics are encountered rarely in studies of students' social networks, leaving researchers with little help in theorizing about how whole-network properties might influence outcomes. Density, modularity, and transitivity are all metrics affected by how ties are recognized, while clustering of academic engagement is directly pertinent to current concerns. It is not expected that the most extreme value of any measurement will indicate the "best" network identification method, but only that these values are partially shaped by how a network is identified.

$R Q$ 3: How do identified networks compare in actor-level metrics?

RQ 3.a: Are there differences in students' number of partners?

$R Q$ 3.b: Are there differences in students' network centrality?

$R Q$ 3.c: Are there differences in students' average path length?

RQ 3.d: Are there differences in students' maximum path length? 
Finding The Missing Links, 81

$R Q$ 3.e: Are there differences in the average correlation between students' academic engagement and the average engagement of their partners?

Actor-level metrics tend to be encountered more often than whole-network metrics in school studies, but they were rarely found in the network comparisons, leaving questions about what these different networks imply about students. Are measurements of students' position in the network largely unaffected by identification method? If so, worrying about the merits of identification methods may be unnecessary. However, if measurements of students' position are greatly affected by identification method, this may influence future research in how student position is related to outcomes.

$R Q$ 4: How do identified networks compare in analyses of selection and influence processes for changes in individual academic engagement across the school year?

Finally, to examine a question of substantive importance, selection and influence processes for changes in individual academic engagement as a function of peers' engagement will be examined. The question of whether peers can influence the academic engagement of one another, or whether students simply select peers with similar levels of engagement, is an important one for school researchers. If peers influence one another, interventions can be designed to capitalize on this. If they do not, such attempts would theoretically be in vain. To determine whether the manner in which the network is defined influences estimates of selection and influence, analyses of these processes will be conducted for each network and compared. 
Finding The Missing Links, 82

CHAPTER 4: Method

The data for this study were collected as part of a larger longitudinal project (see Kindermann, 2007; Skinner, Zimmer-Gembeck \& Connell, 1998). Kindermann's study on peer groups focused on the entire cohort of 366 sixth-graders in a rural/suburban town in a northeastern state (2007). The town had a population of approximately $15,000,90 \%$ were of European-American descent, and about $87 \%$ of adults had a high-school diploma or higher degree. The school examined was the only one for this age group in the town. The closest other middle school was 16 miles away. Thus, a small group of students who commuted to school outside of town or attended private school were likely not included in the original study.

\section{Setting and Sample}

A total of 366 sixth graders (48\% female) attended the school. From these, 340 (93\%) participated with consent and parental permission. Information about ethnic background was not obtained. The school was organized in homerooms; students were grouped into 13 homeroom classrooms in which they had at least one lesson with their homeroom teacher every day. Students moved to other classrooms for several of their other lessons. The school's intent was that each classroom had one teacher who was primarily responsible for the students and who saw them every day. All 13 homeroom teachers participated in the study and stated that they were very familiar with their students. 
Finding The Missing Links, 83

\section{Design and Measures}

Student questionnaires, teacher reports, and student file data were used in the study. Student questionnaires were administered at two time points during regular classroom hours: The first measurement point was within the first three months of the school year (fall); the second was within three months of its end (spring). The student questionnaires consisted (in addition to other measures not described here) of demographic information, a 14-item scale assessing students' academic engagement and disaffection in the classroom, questionnaires about levels of parents' and teachers' involvement with students (Kindermann, 2007), and an assessment of students' peer groups. Teacher reports focused on students' academic engagement in their classrooms and were collected within a three-month time window around the student data collections. In addition, information about students' grades and achievement was obtained.

Teacher-reports of engagement. To measure academic engagement, a 14-item scale that tapped teachers' perception of each student's engagement was used (Wellborn, 1991). The measure consists of two components: emotional (ex. "In my class, this student appears happy") and behavioral engagement (ex. "This student works as hard as he/she can") rated on a likert-type scale from one to five. Previous research (Wellborn, 1991) indicated that these components are moderately correlated $(r=.32, n=144)$ and form an internally consistent indicator of engagement $(\alpha=.95, \mathrm{n}=185)$.

At the beginning of the sixth grade, teachers provided engagement ratings on 318 students (representing $87 \%$ of the population, and $94 \%$ of the initially enrolled students). 
Finding The Missing Links, 84

Eight students were missed because they had recently enrolled and the teachers did not know them well, seven students changed homerooms, and seven other students had not yet arrived at school. By the end of the school year, reports were obtained for 322 students (18 of the fall students had left, but 22 students who were missed in the fall were included). Teacher-reports were available for 300 students at both time points.

Peer groups. Students' descriptions of their observations of peer groups in their grade were obtained via SCM. The entire grade was targeted (and not just classrooms) because in the sixth grade, students moved across classrooms during the day.

In the questionnaires, students were asked to list groups of others in their grade whom they knew to frequently "hang out" together. Students were asked to list as many members as they knew of (in school and outside), as many groups as they knew of, to include the same children in multiple groups if appropriate, to include dyads, and to not forget to include themselves in at least one group. The forms provided spaces for up to 20 students and 20 groups (no student used all of the space).

In a typical report, a student might, for example, indicate that students $\mathrm{A}, \mathrm{B}$, and C formed one group, and that students D and E formed a separate group. Students were also asked to provide a name for each group that characterized "what the group was about." This information was requested for descriptive purposes and will not be analyzed. This method relies on free recall and focuses on public knowledge of affiliations. Connections were assumed to exist between students when observers agreed they frequently affiliated together (see "Network identification", next section). 
Finding The Missing Links, 85

At the beginning of sixth grade, 280 students ( $76 \%$ of the population; $56 \%$ female) provided information about peer networks in their grade; although 60 children (18\% of participants; nearly evenly distributed across homerooms and gender) did not (five children had completely illegible entries, 15 indicated they did not know anything about groups, 33 left this part of the questionnaire unanswered, and seven had not yet arrived for the fall term).

Network identification. A typical student's peer group report consists of a series of lists. Each list contains students the respondent believes "hang out" together. The respondent is also requested to provide a list of students he or she "hangs out" with as a group. Thus, the typical student report will consist of peer groups the respondent may be a member of (self-report) and peer groups the respondent may not be a member of (participant observer). See figure 2 for an example (next page). A hypothetical student, Samantha, indicated two groups on her nomination form: in the first group, she indicated that she hung out with Tom, Shawn and Eric. This statement contains two pieces of information: a self-report that she has a tie with Tom, Shawn, and Eric, and a participant observation that Tom, Shawn, and Eric all have ties with one another. In the second group, Samantha indicated that she saw Dan, Brittany, Fred, Elle, Greg and Janet hanging out often, thus proposing a participant observation between all pairs of students in the second group.

Figure 2. An example of a typical student report

\begin{tabular}{|l|l|l|l|l|l|}
\hline Informant: & Samantha & & & \\
\hline Group 1: & Samantha & Tom & Shawn & Eric & \\
\hline Group 2: & Dan & Brittany & Fred & Elle $\quad$ Greg Janet \\
\hline
\end{tabular}


Finding The Missing Links, 86

Broadly, the difference between the participant observer method and the selfreport method is that the self-report method only considers nominations from students to others, whereas this method allows third-parties to propose nominations between students. Basically, this involves using information from informants who are themselves not members of a specific group, in order to identify groups. To construct networks using the participant observer method, participant observations will be processed using a script I have written in R, which will aggregate individual participant observations to form an actor $\mathrm{x}$ actor co-occurrence matrix, listing the number of groups a pair of students were nominated in. Diagonal values will be set to missing. From this matrix, four peer networks will be identified using syntax I have written in R:

1) Reciprocally-nominated friendships: This network will consist of ties between students who nominated one another as friends. Non-reciprocal nominations will not be included.

2) Participant observations using probability scores for creating ties: An unconditional and conditional probability will be calculated for each pairing of students. The unconditional probability will simply be the number of groups a focal student was identified in by informants, divided by the total number of groups identified. The conditional probability will be the number of times a focal partner was co-nominated with the focal student, divided by the number of groups the focal student was nominated in. These two values will be compared with one another via binomial $z$-tests. Only connections that are significant $(p \leq .01)$ will be accepted. When expected cell frequencies are 
Finding The Missing Links, 87

small (less than 5), the binomial $z$-test can be problematic (Haberman, 1988). In these cases, the probability from Fisher's exact test will be calculated as well as the binomial $z$-score, and the connection will be accepted if both statistics are significant. Fisher's exact test relies directly on probabilities instead of $z$-scores and is, thus, non-parametric. Any significant connections based on a single nomination will not be accepted, as these are likely a child's self-nomination.

3) Participant observations using rules for making social groups: This procedure is based on Cairns and colleagues (1991). A matrix of correlations of pairs of students' co-occurrences will be generated, and used to assign students to social groups according to specific rules, as described by Cairns and colleagues (1991): a student will be part of a social group if their peer nominations have a correlation of $r \geq .4$ with at least $50 \%$ of the members of the group, and the correlation is statistically significant $(\alpha=.05)$. The computational work was handled by the SCM 4.0 program (Leung, 1998).

4) Participant observations using factor analysis for making social groups: As described by Gest and colleagues (2007), an unrotated principal component analysis (PCA) will be performed on the matrix of covariances derived from the co-occurrences. Any factor with an eigenvalue greater than 1.0 will be extracted and interpreted as a social group. Then, a varimax rotation will be applied to determine if each factor is identified by at least three students whose primary loading $(>.30)$ is for that factor. If any factors do not meet 
Finding The Missing Links, 88

these criteria, the PCA will be rerun with one less factor. If any students have significant factor loadings on more than one factor, they will be assigned to the factor they load highest on. 
Finding The Missing Links, 89

CHAPTER 5: Results

The analyses proceeded in three steps. First, descriptive statistics of the two collection methods and four network identification methods were inspected. Second, hypotheses relating to the collection methods were assessed, followed by hypotheses relating to the network identification methods. Third, research questions were examined in order. All calculations were carried out using R version 3.2.1 (R Core Team, 2015).

\section{Descriptive Statistics}

Descriptive statistics for collection methods are presented in Table 1, descriptive statistics for network identification methods in Table 2. In the dataset for the fall data collection, 637 total students were included (either as nominees, informants, or both). Of these, 346 were students in the school. In the spring term data, 580 total students were included, of which 346 were students in the sixth grade. Between fall and spring terms, a total of 378 students were included in both datasets, including 334 who were in both terms of the sixth grade. For all subsequent analyses, non-sixth grade students were removed from the datasets and sixth-grade students who did not appear in the social network data (but who did have teacher reports of engagement) were included as isolates.

\section{Collection Method Hypothesis Tests}

To examine the hypotheses, a multilevel logistical regression was used, in which terms of school were nested within students. The dependent variable was a binomial value indicating whether or not a student was covered. This variable was constructed by 
Finding The Missing Links, 90

Table 1. Descriptive statistics for collection methods

\begin{tabular}{lcccc}
\hline & \multicolumn{2}{c}{ Self-Report } & & \multicolumn{2}{c}{$\begin{array}{c}\text { Participant } \\
\text { Observation }\end{array}$} \\
& Fall & Spring & Fall & Spring \\
\hline \# of Informants & 180 & 145 & 190 & 191 \\
\# of Groups & 253 & 188 & 441 & 476 \\
Nominees/Group & 3.98 & 4.50 & 4.05 & 4.46 \\
\% Covered & 82 & 78 & 87 & 91 \\
- Boys & 73 & 73 & 81 & 89 \\
- Girls & 92 & 84 & 93 & 94 \\
Engagement & 3.13 & 3.11 & 3.10 & 3.08 \\
\hline
\end{tabular}

dichotomizing the number of nominations a student had, where zero nominations indicated that a student was not covered (coded as zero), and one or more indicated that they were covered (coded as one). Then, participant observation was included as a dummy variable (where zero indicated that self-report was used, and one indicated that participant observation was used). Interactions were included with the collection method variable as necessary for the hypotheses. These will be discussed in the individual hypotheses.

Whole population. To determine whether the participant observation method covered more students than the self-report method, the dummy variable of collection

Table 2. Descriptive statistics for network identification methods

\begin{tabular}{|c|c|c|c|c|c|c|c|c|}
\hline & \multicolumn{2}{|c|}{$\frac{\text { Probability }}{\text { Score }}$} & \multicolumn{2}{|c|}{ Reciprocal } & \multicolumn{2}{|c|}{$\frac{\text { Factor- }}{\text { Analytic }}$} & \multicolumn{2}{|c|}{ Rule-Based } \\
\hline & Fall & Spring & Fall & Spring & Fall & Spring & Fall & Spring \\
\hline$\%$ Capt & 77 & 80 & 32 & 25 & 69 & 84 & 93 & 94 \\
\hline - Boys & 66 & 74 & 23 & 16 & 65 & 82 & 89 & 92 \\
\hline - Girls & 90 & 87 & 43 & 36 & 72 & 87 & 98 & 96 \\
\hline Engagement & 3.14 & 3.09 & 3.31 & 3.15 & 3.15 & 3.09 & 3.11 & 3.08 \\
\hline
\end{tabular}


Finding The Missing Links, 91

Table 3. Log-odds of being covered, by data collection method

\begin{tabular}{lcrrrr}
\hline & Est. & \multicolumn{1}{c}{ Std. Error } & \multicolumn{1}{c}{$\mathbf{Z}$} & p & \\
\hline (Intercept) & 2.11 & 0.19 & 11.18 & $<.001$ & $* * *$ \\
Partic. Obs. & 0.92 & 0.18 & 5.17 & $<.001$ & $* * *$ \\
\hline$* * * p<.001 ; * * p<.01 ; * \boldsymbol{p}<.05$ & & & & &
\end{tabular}

method was used to predict the log-odds of being covered (see Table 3). This model yielded a significant finding, supporting hypothesis $1(\mathrm{~b}=0.92, p<.001)$. Raising the natural logarithm to the power of the coefficient for the collection method, the odds ratio (OR) for collection method is 2.5 , thus indicating that the average student was two-and-ahalf times as likely to be covered by the participant observation method as by the selfreport method.

Population by gender. Student gender was included as an interaction with collection method, where boys were coded as zero and girls were coded as one (see Table 4). This model yielded a significant finding for boys $(b=0.96, p<.001)$, indicating that the average boy's probability of being covered was greater when the participant observation method was used. In addition, although the proportion of girls covered was significantly higher than the proportion of boys covered $(\mathrm{b}=1.41, p<.001)$, holding collection method constant, the interaction between collection method and student gender

Table 4. Log-odds of being covered, by data collection method and student gender

\begin{tabular}{lcrrrr}
\hline & Est. & Std. Error & \multicolumn{1}{c}{$\mathbf{z}$} & \multicolumn{1}{c}{$\mathbf{p}$} & \\
\hline (Intercept) & 1.47 & 0.20 & 7.35 & $<.001$ & $* * *$ \\
Partic. Obs. & 0.96 & 0.21 & 4.47 & $<.001$ & $* * *$ \\
Female & 1.41 & 0.30 & 4.62 & $<.001$ & $* * *$ \\
Partic. Obs. * Female & -0.11 & 0.37 & -0.30 & 0.76 & \\
\hline
\end{tabular}

$* * * p<.001 ;{ }^{* *} p<.01 ;{ }^{*} p<.05$ 
Finding The Missing Links, 92

was not significant $(p=0.76)$, indicating that neither method was significantly more likely to cover girls. Thus, both elements of hypothesis two were supported: the participant observation method proved more effective than the self-report method at capturing boys $(\mathrm{OR}=2.6)$, and the participant observation method proved equally effective to the self-report method at capturing girls $(\mathrm{OR}=0.90)$.

Population by engagement. Student gender was replaced by student engagement, which was normalized and set as an interaction with collection method. This model (see Table 5) yielded a significant, positive relationship between student engagement and probability of being covered for self-report $(b=0.63, p=0.003)$. In addition, a marginally significant, negative coefficient was found for using the participant observation method $(b=-0.54, p=0.08)$, indicating that while student engagement was positively related to probability of being covered for participant observations, it was marginally less so $(\mathrm{OR}=1.09)$ than for the self-report method $(\mathrm{OR}=1.88)$. As such, hypothesis 3 was supported because engagement had a stronger relationship with whether or not a student was covered for self-report than for participant observations. Thus, the self-report method can be viewed as more biased against students with low levels of engagement than the participant observation method.

Table 5. Log-odds of being covered, by data collection method and academic engagement level

\begin{tabular}{llrrrl}
\hline & Est. & Std. Error & \multicolumn{1}{c}{$\mathbf{z}$} & \multicolumn{1}{c}{$\boldsymbol{p}$} & \\
\hline (Intercept) & 2.07 & 0.18 & 11.58 & $<.001$ & $* * *$ \\
Partic. Obs. & 0.97 & 0.20 & 4.85 & $<.001$ & $* * *$ \\
Engagement & 0.63 & 0.21 & 3.02 & 0.003 & $* *$ \\
Partic. Obs. ${ }^{*}$ Engagement & -0.54 & 0.31 & -1.75 & 0.08 & \\
\hline$* * * \boldsymbol{p}<.001 ; * \boldsymbol{p}<.01 ; \boldsymbol{0}<\boldsymbol{0 5}$ & & & & &
\end{tabular}


Finding The Missing Links, 93

\section{Network Identification Method Hypothesis Tests}

To examine the hypotheses that probability of being covered varied by network identification method, a multilevel logistical regression was again used, in which terms of school were nested within students. The dependent variable was a binomial value indicating whether or not a student was captured. This variable was constructed by dichotomizing the number of partners a student was identified as having, where zero partners indicated that a student was not captured (i.e., an isolate; coded as zero), and one or more indicated that they were captured (coded as one). Then, identification method was included as a nominal variable, where each identification method was coded as a separate factor. For all models, the probability score was the first factor, and so it was the reference method that the other three methods were compared against. Interactions were included with the identification method variable as necessary for the hypotheses. These will be discussed in the individual hypotheses.

Whole population. To determine whether the probability score method captured a greater proportion of students than the other identification methods, the identification method variable was used to predict the log-odds of being captured (see Table 6). This model yielded a negative, significant finding for reciprocal nominations $(b=-3.34$,

Table 6. Log-odds of being captured, by network identification method

\begin{tabular}{lcrrrr}
\hline & Est. & \multicolumn{1}{c}{ Std. Error } & \multicolumn{1}{c}{ z } & \multicolumn{1}{c}{$\mathbf{p}$} & \\
\hline (Intercept) & 1.94 & 0.15 & 12.64 & $<.001$ & $* * *$ \\
Reciprocal Nom. & -3.34 & 0.18 & -18.97 & $<.001$ & $* * *$ \\
Factor-Analytic & -0.18 & 0.15 & -1.21 & 0.22 & \\
Rule-Based & 1.89 & 0.21 & 8.85 & $<.001$ & $* * *$ \\
\hline$* * * \boldsymbol{p}<.001 ; * * \boldsymbol{p}<.01 ; * \boldsymbol{p}<.05$ & & & & &
\end{tabular}


Finding The Missing Links, 94

$p<.001)$, a non-significant finding for factor-analyses $(p=0.22)$, and a positive,

significant finding for rules $(\mathrm{b}=1.89, p<.001)$. Thus, hypothesis 4 was partly supported:

the reciprocal nomination method captured a lower percent of students than the

probability score method, while the factor-analytic method did not differ and the rule

method captured significantly more. Compared to the probability score method, the

reciprocal nomination method was 28.2 times less likely to capture any given student, while the rule method was 6.6 times more likely.

Population by gender. Student gender was added as an interaction with identification method (see Table 7). Differences between identification methods were found for both genders. The reciprocal nomination method was less likely than the probability score method to capture boys $(\mathrm{b}=-3.33, p<.001, \mathrm{OR}=0.04)$, while the rule method was more likely $(\mathrm{b}=1.97, p<.001, \mathrm{OR}=7.2)$, and the factor-analytic method $\operatorname{did}$ not differ $(p=0.16)$.

Holding identification method constant, girls were more likely than boys to be

Table 7. Log-odds of being captured, by network identification method and student gender

\begin{tabular}{lccrrrr}
\hline & \multicolumn{1}{c}{ Est. } & Std. Error & \multicolumn{1}{c}{$\boldsymbol{z}$} & \multicolumn{1}{c}{$\boldsymbol{p}$} & \\
\hline (Intercept) & 1.23 & 0.19 & 6.57 & $<.001$ & $* * *$ \\
Reciprocal Nom. & -3.33 & 0.23 & -14.49 & $<.001$ & $* * *$ \\
Factor-Analytic & 0.27 & 0.20 & 1.39 & 0.16 & \\
Rule-Based & 1.97 & 0.25 & 7.81 & $<.001$ & $* * *$ \\
Female & 1.66 & 0.31 & 5.42 & $<.001$ & $* * *$ \\
Reciprocal Nom. * Female & -0.23 & 0.33 & -0.70 & 0.48 & \\
Factor-Analytic * Female & -1.18 & 0.32 & -3.69 & $<.001$ & $* * *$ \\
Rule-Based ${ }^{*}$ Female & -0.08 & 0.48 & -0.17 & 0.86 & \\
\hline$* * * \boldsymbol{p}<.001 ; * * \boldsymbol{p}<.01 ; \boldsymbol{p}<.05$ & & & & & &
\end{tabular}


Finding The Missing Links, 95

captured $(b=1.66, p<.001)$. Neither the reciprocal nomination nor rule-based methods showed significant deviations from this finding ( $p=0.48$ and $p=0.86$, respectively), but the factor-analytic method $\operatorname{did}(\mathrm{b}=-1.18, p<.001, \mathrm{OR}=0.3)$. Thus, compared to the probability score method, boys were less likely to be captured by the reciprocal nomination method, more likely to be captured by the rule method, and as likely to be captured by the factor-analytic method, supporting the first part of hypothesis 5 somewhat. Meanwhile, although girls were more likely to be captured overall, they were about equally likely to be captured by the probability score, reciprocal nomination, and rule methods, and less likely to be captured by the factor-analytic method, mostly supporting the second part of hypothesis 5 .

As a point of clarification, this is not to say that equal proportions of girls were captured by the probability score, reciprocal nomination, and rule methods, as that would not be true ( $\mathrm{p}_{\text {girls }}=0.88 ; 0.39 ; 0.97$, respectively). Rather, differences in the proportion of girls captured appeared to be primarily due to differences in the proportion of students captured, and each of the three methods appeared to be equally influenced when a student was female. The sole exception is the factor-analytic method, which only differed from the probability score method when students were female. Thus, all network identification methods can be viewed as biased in favor of girls (a student is more likely to be captured if they are female), but being female seems to effect the factor-analytic method less so.

Population by engagement. Student gender was replaced by student engagement, which was normalized and set as an interaction with network identification method. The resulting model (see Table 8 ) revealed a positive relationship between log- 
Finding The Missing Links, 96

Table 8. Log-odds of being captured, by network identification method and academic engagement level

\begin{tabular}{lrrrrr}
\hline & Est. & Std. Error & \multicolumn{1}{c}{ Z } & \multicolumn{1}{c}{ P } & \\
\hline (Intercept) & 2.08 & 0.16 & 13.18 & $<.001$ & $* * *$ \\
Reciprocal Nom. & -3.26 & 0.19 & -17.37 & $<.001$ & $* * *$ \\
Factor-Analytic & -0.20 & 0.17 & -1.19 & 0.23 & \\
Rule-Based & 2.40 & 0.30 & 8.11 & $<.001$ & $* * *$ \\
Engagement & 0.53 & 0.22 & 2.44 & 0.02 & $*$ \\
Reciprocal Nom. * Engagement & 0.25 & 0.26 & 0.96 & 0.34 & \\
Factor-Analytic * Engagement & -0.12 & 0.26 & -0.47 & 0.64 & \\
Rule-Based * Engagement & 0.17 & 0.43 & 0.39 & 0.69 & \\
\hline
\end{tabular}

$* * * p<.001 ; * * p<.01 ; * p<.05$

odds of being captured and engagement $(b=0.53, p=0.02)$, which did not deviate at all across the identification methods $\left(p_{\text {reciprocal nomination }}=0.34 ; p_{\text {factor-analytic }}=0.64 ; p_{\text {rules }}=\right.$ 0.69). Thus, the likelihood of being captured was equally influenced by student engagement for each identification method, contrary to hypothesis 6 .

\section{Agreement Analyses}

To examine the extent that networks overlapped, three calculations that were commonly encountered in network comparisons were employed: percent overlap, correlation, and interrater agreement. Statistical significance will not be examined for any of these parameters because I have no expectations about how they should turn out and can derive no theoretical value depending on how they do.

Percent overlap. Percent overlap was calculated by simply comparing matrices of network ties, in which an existing tie is represented by a one and an absent tie is represented by a zero. When a tie between a pair of students was the same for a particular pair of networks, this was regarded as an agreement, and when they did not match, this 
Finding The Missing Links, 97

was regarded as a disagreement. Then, the percent overlap is simply the number of agreements divided by the number of agreements and disagreements. For results, see Table 9.

To determine to what extent this percent overlap is due to agreements about existing ties and absent ties, I also calculated the percent of ties in one network which were present in another network (this can be thought of as the answer to the question "given Network 1 assigns a tie between students A and B, what is the probability that Network 2 will also assign a tie between students A and B?"), as well as the percent of absent ties in one network which were also absent in another network ("given Network 1 does not assign a tie between students $\mathrm{A}$ and $\mathrm{B}$, what is the probability that Network 2 will not assign a tie between students A and B?"). Thus, for each pair of networks, there are three percent overlap values: the total percent overlap, the percent of ties also present in the comparison network, and the percent of non-ties also present in the comparison network.

Overall, all four networks showed very high levels of overall agreement, at between $97 \%$ - 99\%. However, this was primarily due to agreement over non-ties, which ranged from $97 \%$ - 100\%. In considering agreement between ties, there was far more variability. The reciprocal nomination network generally showed high tie agreement with other networks, with between $79 \%-96 \%$ of its ties being found in the other three networks, while the rule-based network generally showed low tie agreement with the others, with between $5 \%$ and $51 \%$ of its ties being found in the other networks. Both the probability and factor-analytic networks showed a very high variation of tie agreement, 
Finding The Missing Links, 98

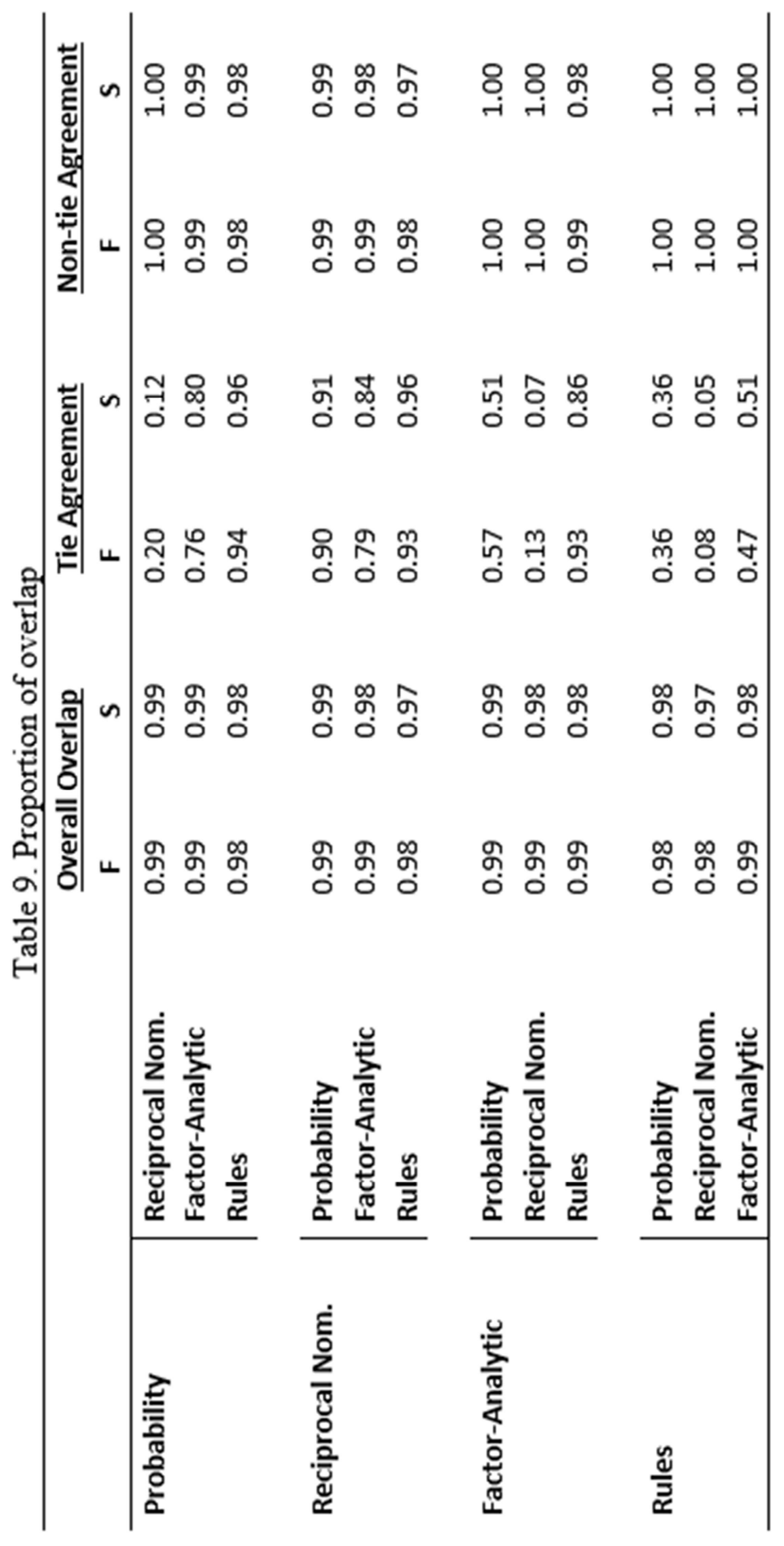


with a tie agreement rate of between $12 \%-96 \%$ and $7 \%-93 \%$, respectively.

The rate of tie agreement showed a great deal of stability from fall to spring term, changing from $0 \%-8 \%$. In addition, rank order was maintained in all cases (in other words, for a given network, the networks its ties agreed with most and least in fall term were the same networks it agreed with most and least in spring term).

Most tie agreements were fairly one-sided, in that while one network may have shown a high level of tie agreement with another network, the level of tie agreement was generally poor if the order of the networks was flipped. The most two-sided level of tie agreement was found between the probability and factor-analytic networks, with $76 \%$ and $80 \%$ of the probability network's ties being found in the factor-analytic network (for fall and spring, respectively), and with $57 \%$ and $51 \%$ of the factor-analytic network's ties being found in the probability network.

This method does not take chance agreement into account, but calculations using the binomial distribution indicated that the probability of any two pairings of networks to agree upon at least as many ties as were observed was essentially zero.

Correlation. Pearson's correlations were computed for networks. For a breakdown, see Table 10. As a measure of agreement, researchers do not recommend using percent overlap and correlations (Hunt, 1986). Percent overlap does not take chance agreement into consideration, meanwhile correlations are unaffected by systematic biases. Nonetheless, these measures were computed for comparability with previous networks comparison studies. 
Finding The Missing Links, 100

Table 10. Correlations for identified networks*

\begin{tabular}{lcccc}
\hline & Probability & Reciprocal Nom. & Factor-Analytic & Rules \\
\hline Probability & - & 0.42 & 0.65 & 0.58 \\
Reciprocal Nom. & 0.33 & - & 0.32 & 0.26 \\
Factor-Analytic & 0.64 & 0.24 & - & 0.66 \\
Rules & 0.58 & 0.21 & 0.65 & - \\
\hline
\end{tabular}

* Fall-term is above diagonal, spring-term below

Overall, the factor-analytic network showed the strongest correlations with the other networks, with a correlation of $0.65 / 0.64$ with the probability network (fall/spring, respectively), $0.32 / 0.24$ with the reciprocal nomination network, and $0.66 / 0.65$ with the rule-based network. The probability network was close behind, with correlations of $0.42 / 0.33$ with the reciprocal nomination network and $0.58 / 0.58$ for the rule-based network. Finally, the weakest correlation was between the rule-based and reciprocal nomination networks, at $0.26 / 0.21$, while the strongest was when the factor-analytic network was compared with either the rule-based or probability networks. Generally, the reciprocal nomination network demonstrated the weakest correlations.

Interrater agreement. Interrater agreement was assessed via Fleiss' $\kappa(1971)$, as recommended by Hunt (1986) for binary ratings (in this case, for unweighted, undirected ties). The results of these analyses largely mirrored the results of the correlations (see Table 11). The factor-analytic network, as before, had the strongest interrater agreements, at $0.65 / 0.62$ with the probability network in fall/spring, $0.22 / 0.12$ with the reciprocal nomination network, and $0.62 / 0.63$ with the rule-based network. The probability network, again, was close behind, with interrater agreements of $0.32 / 0.21$ with the reciprocal nomination network and $0.51 / 0.51$ with the rule-based network. Finally, the reciprocal 
Finding The Missing Links, 101

Table 11. Interrater agreements for identified networks*

\begin{tabular}{lcccc}
\hline & Probability & Reciprocal Nom. & Factor-Analytic & Rules \\
\hline Probability & - & 0.32 & 0.65 & 0.51 \\
Reciprocal Nom. & 0.21 & - & 0.22 & 0.13 \\
Factor-Analytic & 0.62 & 0.12 & - & 0.62 \\
Rules & 0.51 & 0.08 & 0.63 & - \\
\hline
\end{tabular}

* Fall-term is above diagonal, spring-term below

nomination network had an interrater agreement of $0.13 / 0.08$ with the rule-based network, the weakest pair of interrater agreements by far. Meanwhile, comparing the factor-analytic with the rule-based and probability networks, again, yielded the strongest pair of agreements.

\section{Whole-Network Metrics}

To better understand the structural differences between the four social networks, whole-network metrics were computed and compared. As with the agreement analyses, no tests of statistical significance were performed between networks. The following metrics were calculated for each network: density, modularity, transitivity, and clustering of academic engagement. Except when otherwise specified, calculations were performed using the 'igraph' package in R (Csardi \& Nepusz, 2006). Sample statistics are presented in Table 12, effect sizes of pairwise comparisons in Table 13.

Density. This measure indicates the number of existing ties divided by the number of possible ties. The rule-based network was the most dense, with $5.1 \%$ of possible ties present in the fall, and $6.1 \%$ in the spring. The reciprocal nomination network was least dense, with $0.4 \%$ of ties present in the fall, and $0.3 \%$ present in the spring. Absolute values of effect sizes of pairwise comparisons between the densities of 
Finding The Missing Links, 102
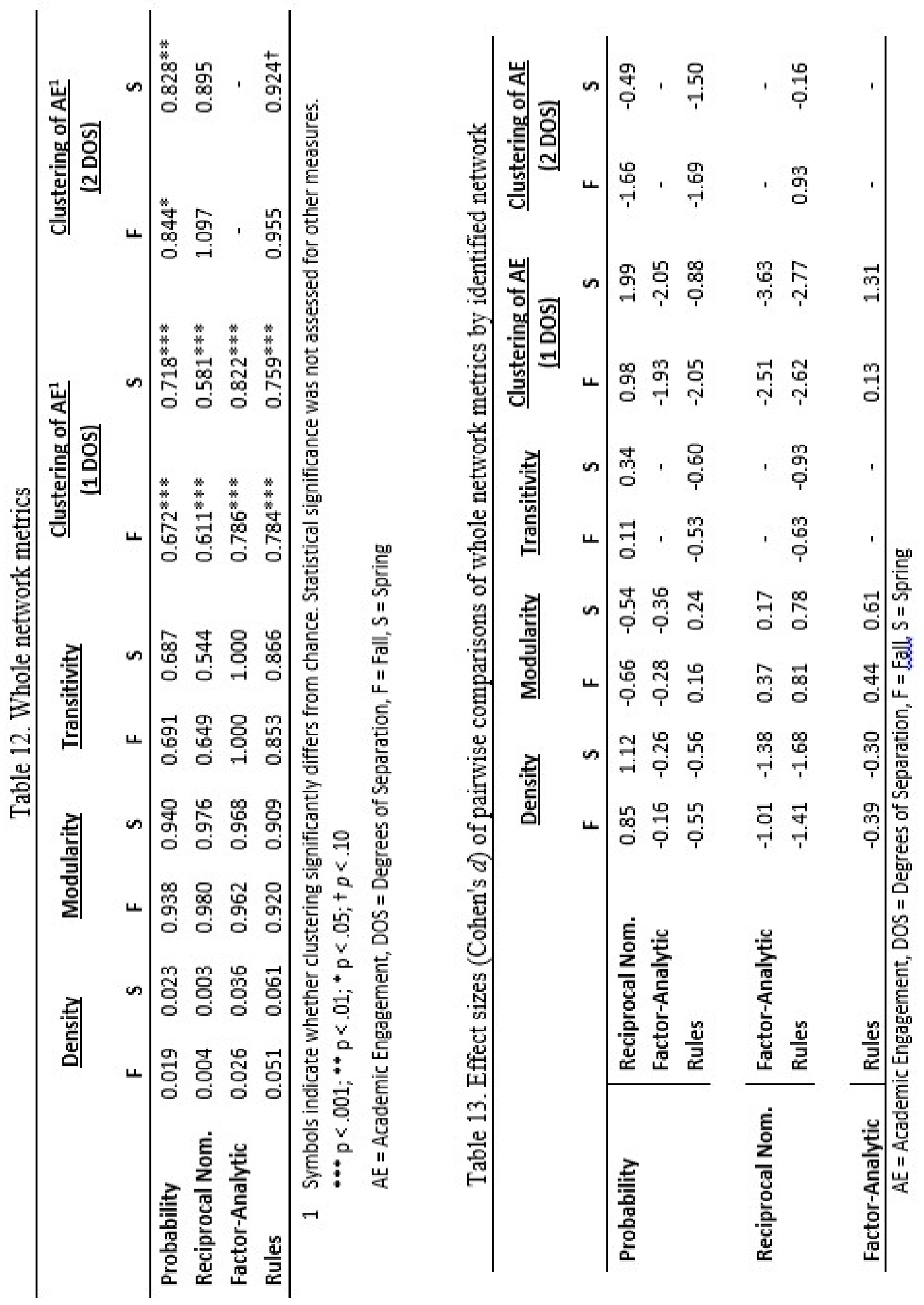
Finding The Missing Links, 103

networks averaged $\bar{d}=0.81$, with the smallest effect sizes being the ones comparing the densities of the probability and factor-analytic networks, and the largest being between the reciprocal nomination and rule-based networks.

Modularity. Modularity indicates the extent to which a network is comprised of a series of communities in which members are densely interconnected, while being only sparsely connected with non-members. The algorithm used to determine modularity scores began by partitioning the network into a series of non-overlapping subsets, then determined what proportion of ties existed within those subsets as the modularity score. Thus, although the factor-analytic network was comprised of a small set of maximally dense, non-overlapping groups, and should, logically, have a modularity score of 1 , the algorithm partitioned the network in such a way that some ties were left out of partitions. Thus, whether the modularity score should be the one provided by the algorithm or the one that may seem more logical is a consideration to be made by the reader. To be consistent with the other modularity score measures, I have chosen to report the score provided by the algorithm.

The modularity scores were fairly similar between all networks, with relatively small absolute effect sizes $(\bar{d}=0.45)$.

Transitivity. Transitivity indicates the probability that two partners of a given student are, themselves, connected. For this measure, the factor-analytic network had the highest score, with a perfect 1.0 in the fall and spring terms, no doubt due to the construction of this network. The other group-based network, the network identified using rules, had a fairly high transitivity score, at $0.853 / 0.866$ in the fall/spring terms, 
Finding The Missing Links, 104

respectively. The probability network had transitivity scores of $0.691 / 0.687$, while the reciprocal nomination network was close behind at $0.649 / 0.544$. Interestingly, the reciprocal nomination network's transitivity score showed a $16 \%$ drop from the fall to spring term. Absolute effect sizes of pairwise comparisons of networks were small to moderately large, averaging $\bar{d}=0.52$. In all cases, the magnitude of the effect size significantly increased from fall to spring.

Clustering of academic engagement. The clustering of academic engagement refers to how similar students' level of engagement is to their partners'. This was calculated through a Monte Carlo-style bootstrapping method, adapting a procedure described by Christakis and Fowler (2013): first, the squared difference between students' engagement and the engagement of their partners were determined (the “observed student-partner variance”). Second, engagement scores were randomly assigned around the network and squared differences were calculated again (the "null student-partner variance"). These random assignments and calculations were performed 1,000 times, yielding a series of independent student-partner variances. After each bootstrapping, the observed student-partner variance was divided by the null studentpartner variance to arrive at a variance ratio. After the whole process was performed, there were a distribution of ratios.

The "clustering coefficient" was the average of these ratios. If the clustering coefficient was less than one, this indicated that the engagement of students' partners was closer to their own than average (and is what is expected under clustering). Meanwhile, if 
Finding The Missing Links, 105

the ratio was greater than one, this meant that the engagement of students' partners was more different from their own than average (such a finding is generally not expected).

Following Christakis and Fowler's (2013) findings of clustering to several degrees, separate clustering coefficients were computed for students and their partners of up to three degrees of separation. Each coefficient was subjected to a separate hypothesis test to determine if it significantly differed from one. Because students in the factoranalytic networks could not have partners of two or more degrees of separation, the additional clustering coefficients were calculated only for the other three.

All networks displayed highly significant clustering at one degree of separation in both the fall and spring terms (thus, students' AE levels were closer to their partners' than to random students). In addition, the probability network displayed significant clustering at two degrees of separation for the fall and spring term (thus, students' AE levels in the probability network were closer to the levels of partners of partners than to random students). The rule-based network also may have displayed clustering in the spring term, but definitely not in the fall. No network displayed significant clustering to three degrees of separation.

Pairwise comparisons of network clustering displayed very strong absolute effect sizes at one degree of separation, averaging $\bar{d}=1.90$. At two degrees of separation, absolute effect sizes were smaller, but still quite large, averaging $\bar{d}=1.07$. 
Finding The Missing Links, 106

\section{Actor-Level Metrics}

In addition to network-level metrics, actor-level metrics were computed as well. The following measures will be examined on bootstrapped networks using paired comparisons: average degree, average eigenvector centrality, average path length, average maximum path length, and average correlation between academic engagement of students and their partners. Degree, eigenvector centrality, path length, and maximum path length were calculated for each student using the igraph package in R. Sample statistics are presented in Table 14.

In addition, as metrics were at the level of the actor, the contribution of the network identification method could be determined as the percent of variation in actorlevel scores accounted for by the identification method. For each metric, this value was determined by constructing two multilevel models: a null model, in which values of the metric under study was predicted by the intercepts of students in the networks. In a second model, identification method was included as a predictor. In both models, student ID was used as a nesting variable. The error variance of the second model was divided by the error variance of the null model, and the quotient was subtracted from one to arrive at the proportional reduction in error attributed by the network identification method $(\mathrm{Xu}$, 2003).

Degree. Students' average degree (the average number of other students they were identified as connected to) showed a substantial amount of variation across the identification methodologies. On the one hand, the reciprocal nomination network found only 3.45/3.05 partners per student in the fall/spring terms, respectively, while the rule- 
Finding The Missing Links, 107

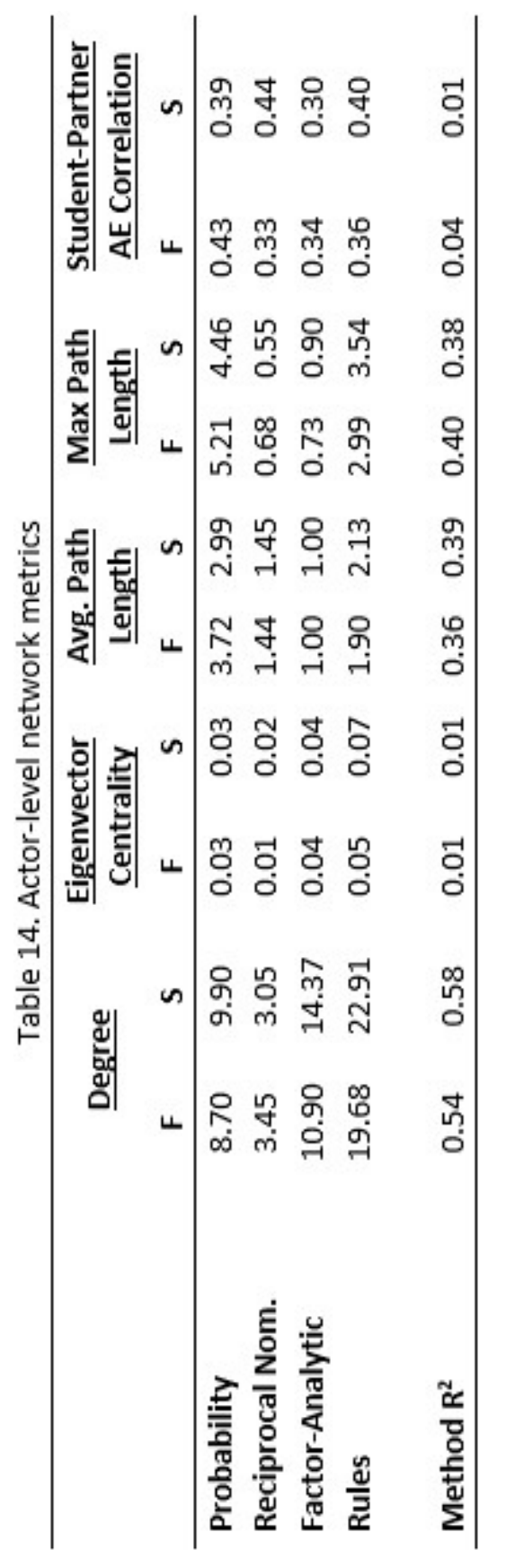


Finding The Missing Links, 108

based network identified 19.68/22.91, or between $5.7-7.5$ times more partners. In addition, between $44-50 \%$ of the variation in degree was accounted for by identification method.

Eigenvector centrality. Under eigenvector centrality, the centrality of a student is influenced by who they are connected to. Thus, a student connected to many other highly central students is considered to be more central than a student connected with many other less central students. Conversely to degree, this value was not heavily influenced by network identification method, with method accounting for about one percent of variation.

Average path length. A student's average path length is the average of the lengths of the shortest paths from that student to any other student in the network (whom they could connect with). Thus, this measure serves as an examination of how wellconnected the overall network is. The largest average path lengths were found in the probability network (3.72/2.99), while the shortest were found for the factor-analytic $(1.00 / 1.00)$. Network identification method accounted for about $33 \%$ of variation in values of average path length.

Max path length. A student's max path length is simply the length of the longest path amongst others that can be reached. The average max path length was highest for the probability network (5.21/4.46) and lowest for the reciprocal network $(0.68 / 0.55)$. Comparing the average and max path lengths, it may be counterintuitive that the reciprocal network has shorter max path lengths than average path lengths, but this is due to how the algorithms are designed. Specifically, the algorithm for max path length takes 
Finding The Missing Links, 109

isolates into consideration (who have a max path length of 0 ), while the algorithm for average path length does not (isolates are simply omitted). Network identification method accounted for about $35 \%$ of variation in these values.

Student-partner correlation of academic engagement. Conceptually similar to clustering of $\mathrm{AE}$ to one degree of separation, the average correlation between students and the averaged engagement of their partners was highest for the probability network $(0.43 / 0.39)$ and lowest for the factor-analytic network (0.34/0.30). Network identification method accounted for very little variance, at most 3\%.

\section{Changes in Academic Engagement by Peer Group}

Peer association for changes in academic engagement from fall to spring was assessed via the actor-partner interdependence model (Cook \& Kenny, 2005), which allowed for simultaneous control and estimation of selection and influence processes. In this model, a structural equation model was defined in which individual engagement and engagement of the individual's peer group in fall were used to predict individual and peer group engagement in spring. A path between the fall-term variables was allowed to account for the correlation between them, and then individual engagement in fall was used to predict individual engagement in spring (thus, by holding fall engagement constant, the coefficient for spring engagement represented the change in engagement across the school year) and peer group engagement in spring (modeling selection processes). Meanwhile, peer group engagement in fall was used to predict peer group engagement in spring (thus, the change in peer group engagement across the school year) 
Finding The Missing Links, 110

and individual engagement in spring (modeling influence processes). This model was constructed for each of the four identified networks. The $\mathrm{R}^{2}$ for each model was determined first by constructing a "null" model, in which selection and influence effects were not estimated. The $\mathrm{R}^{2}$ for this model was compared with the $\mathrm{R}^{2}$ for the full model, with the difference being the $\mathrm{R}^{2}$ for the inclusion of selection and influence estimates.

See Table 15 for influence and selection estimates. In the probability network, a highly significant selection effect was detected $(b=0.19, p<.001)$, as well as a potentially significant influence effect $(b=0.11, p=.08)$. The inclusion of these terms accounted for $2.9 \%$ of the variance in change in individual engagement from fall to spring. In the reciprocal nomination network, no significant selection or influence effects were detected ( $p=.95$ and $p=.41$ respectively) and the model accounted for very little variation $\left(\mathrm{R}^{2}=0.005\right)$. The factor-analytic network yielded only a marginally significant influence estimate $(b=0.14, p=.09)$, and a slight gain in accounting for variation $\left(\mathrm{R}^{2}=0.012\right)$. Finally, a highly significant selection $(\mathrm{b}=0.13, p=.002)$ and marginally significant influence $(b=0.10, p=.09)$ effect were uncovered from the rule-based network, which accounted for a small amount of variation $\left(\mathrm{R}^{2}<0.022\right)$.

Table 15. Estimates of selection and influence by network identification method

\begin{tabular}{lccc}
\hline & $\begin{array}{l}\text { Selection } \\
\text { Estimate }\end{array}$ & $\begin{array}{c}\text { Influence } \\
\text { Estimate }\end{array}$ & $\mathbf{R}^{\mathbf{2}}$ \\
\hline Probability & $0.19^{* * *}$ & $0.11^{\dagger}$ & 0.029 \\
Reciprocal Nom. & -0.01 & -0.12 & 0.005 \\
Factor-Analytic & 0.04 & $0.14^{\dagger}$ & 0.012 \\
Rules & $0.13^{* * *}$ & $0.10^{\dagger}$ & 0.022 \\
\hline$* * * \mathrm{p}<.001 ; * * \mathrm{p}<.01 ; * \mathrm{p}<.05 ;+\mathrm{p}<.10$ & &
\end{tabular}




\section{CHAPTER 6: Discussion}

In this thesis, I had three sets of research objectives. The first set of objectives was to compare hypotheses regarding two methods for collection of social networking data, the second set was to compare hypotheses regarding four methods for identifying social networks, and the third set was to compare properties of the four network identification methods. I will discuss each set of objectives in turn.

\section{Data Collection Method Hypotheses}

The first set of objectives involved testing my prediction that participant observations were a more inclusive, less biased, method of collecting social networking data than the more commonly used self-reports. To determine this, I investigated three hypotheses: the first hypothesis was that participant observations would cover a greater proportion of the student body than self-reports, a hypothesis which was supported by the finding that participant observations were significantly more likely to cover students than self-reports were.

In the second hypothesis, I examined who was covered by gender, expecting that more boys would be captured by the participant observation method than by the selfreport method, while the percent of girls who were captured would be even across the methods. Both expectations were supported, with participant observations being 2.6 times as likely to capture boys as the self-report method, but showing no statistically significant sensitivity toward girls. 
Finding The Missing Links, 112

In the third hypothesis, I examined the contribution of academic engagement toward being covered. This was an important question, because if being disaffected rendered a student less likely to even appear in the social networking data, then resulting social networks would present a skewed picture of the social relations of students with low academic engagement, thus muddying the waters: are disaffected youths this way because they lack social relations, or do they lack social relations because social networking models are biased to depict them as such? I hypothesized that academic engagement (as rated by teachers) would be less predictive of whether or not a student was covered for participant observations than for self-reports, an assertion for which I found a moderate amount of support. Controlling for collection method, each standarddeviation increase in student engagement from mean levels increased a student's probability of being covered. When considering just participant observations, this relationship showed a marginally significant decline.

As such, all hypotheses were met for the first set of research objectives, concerning the data collection methods. Compared to self-reported groups, the participant observation method proved to be more inclusive overall, more inclusive of boys especially, and showed a weaker bias against students with low levels of academic engagement.

\section{Network Identification Method Hypotheses}

In the second set of objectives, I tested four network identification methods: networks identified using probability scores between students, networks identified using 
Finding The Missing Links, 113

reciprocal nominations, networks identified using factor-analytic methods designed to produce non-overlapping groups, and networks identified using the rules developed by Cairns and colleagues. Across these four identification methods, I hypothesized that the probability score approach would be more inclusive of students (especially boys), and least likely to categorize students with low academic engagement as isolated (similar to the first set of objectives).

The first hypothesis, that the network identified by probability scores would capture the largest percent of the student body (i.e., would report the largest percent of students as having at least one partner) was not completely supported. The rule-based network had the largest capture rate, as it was 6.6 times as likely as the probability network to capture students. Meanwhile, the reciprocal nomination network had the lowest capture rate, as it was 28.2 times less likely than the probability score network to capture students. Finally, the factor-analytic network was even with the probability score network.

Under the second hypothesis, I predicted that, of the network identification methods, the probability score network would be most likely to capture boys. In addition, while girls would be more likely than boys to be captured, I predicted that none of the network identification methods would differ in their sensitivity toward girls. This hypothesis was partially supported: girls were generally more likely than boys to be captured, a finding which was uniform across the network identification methods, with the exception of the factor-analytic network (which was less likely than the other network identification methods to capture girls). For boys, the factor-analytic network did not 
Finding The Missing Links, 114

differ from the probability score network in likelihood of capturing boys, while the rulebased network was significantly more likely to capture them, and the reciprocal nomination network was significantly less likely. However, although the networks some differences in sensitivity toward gender, most of the differences in the percent of students captured appeared to be centered on differences in the methods themselves, not so much in how they related to boys and girls.

Finally, in the third hypothesis of the second set of research objectives, I predicted that the probability score network would be least influenced by the academic engagement of students in whether or not students were captured, a prediction which was not supported: academic engagement significantly influenced whether students were captured with no significant differences across all four network identification methods.

Thus, on the surface, the rule-based network would appear to be the most inclusive network, as it captured the greatest proportion of students, showed the expected bias in favor of girls, and did not differ from the other networks in the influence of academic engagement toward likelihood of being captured. However, there is reason to call the performance of the rule-based network into question. Specifically, if the twentyone sixth-grade students who did not appear in the data at all for either term are removed from the rule-based network (which would be fair. Without the self-reported data of those students it cannot truly be demonstrated that they are isolated), then the rule-based network identified only two isolated students in the fall-term and none in the spring-term. This conclusion seems overly generous, as research commonly indicates that $4-10 \%$ of students in primary classrooms lack friends/partners (Doll, 1996). As these data were 
Finding The Missing Links, 115

cross-classroom, it is very possible that the rate of isolated students may be lower by allowing a wider range of nominations. Unfortunately, of the few social networking studies using participant observation methods that allowed for cross-classroom nominations that I could find, only one remarked on number of isolates: Cairns and colleagues' interviews of 80 middle-school children (1985), in which children were specifically asked if there were any students who did not "hang around" with anyone. Out of the 80 interviewees, two children were widely agreed upon as being isolates (for a rate of $2.5 \%$ ). Unfortunately, this study differs from the present in many important ways: children were interviewed (not surveyed), and they were asked specifically about isolated students (isolate-status was inferred in this study).

In addition, this would not be the first time this method's results have been questioned. In their study of the social networks of 190 students (spread across nine Dutch primary schools), Pijl and colleagues (2011) compared the results of the rule-based approach with that of reciprocal nominations. Whereas $5.8 \%$ of the students were completely isolated by the reciprocal nomination method, none were by the rule-based method, making the authors skeptical that the method might be overly generous. Thus, the authors performed a post-hoc analysis of changing the cutoff for correlations from 0.40 to 0.70 , leading to an increase in correspondence between the two methods.

Thus, based on the extant research, the rule-based approach appears to be overly liberal, with a high amount of false positives. There are some possibilities for why this could be. First, as suggested by Pijl and colleagues, the correlation cutoff of 0.40 may be too liberal (2011). No justification for it has ever been given, neither mathematical, 
Finding The Missing Links, 116

theoretical, nor empirical. As such, a higher cutoff may be called for. Second, in this study, students were allowed to nominate partners from out of school, but these nominations had to be cut to bring the number of nominees in line with the requirements of the SCM program (which has a limit of 400 nominees). Perhaps, even if very few of these out of school nominees would have been identified as partners, the extras were necessary to introduce a certain amount of noise that would prevent certain, weaker, connections from being identified as significant. It is possible that, if students were only allowed to nominate others from within the school, their increased focus on intra-school connections might introduce the necessary amount of noise to prevent baseless connections from being formed.

Contrary to the rule-based method, the reciprocal nomination method appeared to be overly conservative. This method revealed isolation rates of $68 \%$ in the fall and $75 \%$ in the spring, which appears to be questionable on the surface. Past research using this methodology around this age group has not uncovered rates near these. For example, Ennett and Bauman (1993) measured the self-reported friendship networks of 1,092 ninth graders in five schools in North Carolina, finding isolate rates of between $17.5-38.2 \%$ (weighted average of 28.6\%). Abel, Plumridge, and Graham measured the self-reported friendship networks of 279 year 10 students in New Zealand, uncovering an isolate rate of $27 \%$ (2002). Finally, Fang, Li, Stanton, and colleagues measured the self-reported friendship networks of 1040 sixth, eighth, and tenth grade students in Beijing, China, finding isolate rates of $38 \%, 50 \%$, and $72 \%$ for each of the three grades, respectively (2003). In all three studies, NEGOPY was used to categorize students into either group 
member, liaison, or isolate. It is worth noting that this program differentiates between three types of isolates: those who are truly isolated ("type 1 isolate"), those with only one connection ("type 2 isolate"), and people connected as a "tree node" (Richards, 1995). Thus, as the definition of isolate for this study only includes those that NEGOPY would designate as a "type 1 isolate", the isolate rates cited above can be considered inflated, relative to this study, and that the actual isolate rates would be even lower than those presented.

The performance of the reciprocal nomination method is anomalous compared to past research, which has made extensive use of this method. The most likely reason for the discrepancy between the results of the method in this study compared to other studies is that, for this study, self-reported friendship data were not collected, but were instead inferred from participant observation data when the informant placed themselves within a group of nominees. Although students were specifically asked to include a group with them in it, respondents may have been too preoccupied with thinking of groups of other students that they simply forgot to nominate themselves.

Finally, it is interesting to note that, despite their mathematical and conceptual differences, the probability score and factor-analytic methods only differed when considering the probability of girls appearing in the network, where the factor-analytic method was significantly less likely to capture girls than the probability score method. 
Finding The Missing Links, 118

\section{Network Identification Method Properties}

In the third set of research objectives, I compared the properties of the networks resulting from each of the four network identification methods. Properties consisted of measures of network agreement, network-level metrics, actor-level metrics, and estimates of selection and influence in changes of student academic engagement across the school year.

Network agreement. The three measures of agreement largely showed the same pattern of findings. Examining the percent overlap indicated that the rule-based network contained the greatest proportion of ties from other networks found in it (containing between $86-96 \%$ of the ties found in the other networks), but had a very low proportion of ties found in other networks (with between $5-51 \%$ of ties from the rule-based network being found in the other networks). Conversely, the reciprocal nomination network contained the smallest proportion of ties from other networks $(5-20 \%)$, but had a very high proportion of its ties appearing in other networks $(79-96 \%)$. These unbalanced matches are no doubt due to the amount of ties in each network. With so many ties in the rule-based network, a large amount of ties from the other networks should appear due to chance alone, while the other networks should lack the ties necessary to agree very much with the rule-based network. Similarly, with so few ties in the reciprocal nomination network, it is expected to see ties from the reciprocal nomination network appearing in the other networks, but not expected to see ties from the other networks appearing in the reciprocal nomination network. Out of all of the pairs of 
Finding The Missing Links, 119

networks, the probability score and factor-analytic networks showed the most balanced tie-agreement, suggesting that these networks were most similar.

Nonetheless, in examining the instances of high tie-agreements for the rule-based and reciprocal nomination networks, it is interesting to note that the observed tieagreements are much higher than would be expected by chance, indicating these networks may represent different layers of the social environment. The reciprocal nomination network might represent the closest connections, followed by the probability and factor-analytic networks, which might consist of both the closest connections and looser ones. Finally, the rule-based network might be made up of the previous connections as well as the loosest, most casual social connections. Thus, it is possible that each of the networks may have something to contribute in identifying certain types of ties. This is a proposal for future researchers.

Like the percent overlap, the correlation and kappa calculations showed a similar pattern of findings. The rule-based and reciprocal nomination networks showed a fairly low rate of agreement with the other networks, while the probability score and factoranalytic networks generally showed the best agreement.

Hence, in spite of warnings, there appears to be little difference between the correlation and kappa calculations, and their pattern of findings is generally fairly similar to the percent overlap. As such, based on the evidence, I suggest using tie-agreement in percent overlap, as it provides the most detail and is easy to interpret.

Network-level metrics. Generally, the rule-based and reciprocal nomination networks were most extreme in terms of whole-network metrics. The rule-based network 
Finding The Missing Links, 120

had the highest density, smallest modularity score, and highest transitivity (behind the factor-analytic network, whose transitivity was 1.0 by design). Conversely, the reciprocal nomination network had the smallest density, largest modularity, and lowest transitivity.

There is a particularly interesting finding regarding clustering of academic engagement. All networks showed significant clustering of academic engagement at one degree of separation, which is to be expected, but only the probability score network showed significant clustering at two degrees of separation, meaning that the academic engagement of partners of partners was closer to one another than would be expected by chance. Due to the construction of the factor-analytic network, it was impossible to assess clustering at more than one degree of separation.

Finally, examining the effect sizes, it is apparent that network identification method has a large impact on density and clustering of academic engagement, and relatively little impact on modularity and transitivity.

Actor-level metrics. As with the network-level metrics, the actor-level metrics generally showed the rule-based and reciprocal nomination networks as occupying the most extreme values, and the probability-score network as occupying the middle-ground. In addition, the probability-score and factor-analytic networks were most similar for the degree and eigenvector centrality measures (due to its group-based nature, the average path length and max path length measures are foregone conclusions for the factoranalytic network, so they are not especially meaningful measures for this network identification method). 
Finding The Missing Links, 121

Finally, examining the percent variation accounted for by network identification method, three metrics were highly influenced by the network identification method:

degree, average path length, and max path length. Meanwhile, eigenvector centrality and the correlation between the academic engagement of students and partners was largely unaffected by how networks were identified.

Changes in academic engagement by peer group. The effect of network identification method on estimates of selection and influence on changes in academic engagement across the school year was assessed. Of these models, the probability score and the rule-based networks yielded similar influence and selection estimates, with even similar levels of significance and percent variation accounted for. The factor-analytic network did not uncover a significant selection effect, but, as with the probability score and rule-based networks, did uncover a marginally significant influence effect. Finally, the reciprocal nomination network failed to uncover a significant selection or influence effect.

It is telling that two of the models (from the probability score and rule-based networks) agreed with one another, even down to the significance levels, suggesting a consistent selection and somewhat consistent influence effect in the data. The reciprocal nomination network may have been too small to detect anything (both in terms of the number of students with at least one partner in the network and the number of partners each student had), while the factor-analytic network may have lacked the structure to allow for proper identification of partners, rendering the model underpowered, at least to detect selection effects. 
Finding The Missing Links, 122

Thus, all hypotheses were supported for the participant observation method, demonstrating its advantage over the self-report method. In addition, findings were somewhat mixed for hypotheses regarding network identification methods, but these may be partly due to an overly liberal approach (the rule-based method) and an approximation of another approach which may have made it too conservative (the reciprocal nomination method). However, the general correspondence between the probability score and the factor-analytic methods in the capture rates, effect of academic engagement on being captured, and tie-agreements suggest that both methods may be somewhat close to capturing the true, underlying, social network.

In addition, the whole-network and actor-level metrics revealed some interesting properties of the probability score network. Compared to the other networks, the probability score network tended to occupy a middle ground in its structural properties. However, one area it shone was in the clustering of academic engagement, where it was the only network to demonstrate significant clustering at one and two degrees of separation. This sensitivity may have also been reflected by the estimates of selection and influence in modeling changes in individual and partners' academic engagement over the school year, leading to a model that demonstrated significant selection effects and marginally significant influence effects. The fact that the rule-based network produced a highly similar model supports the accuracy of the probability score network's model.

Thus, there are a lot of reasons to recommend the probability score method over the others. It yields networks with reasonable structural properties, which captures 
Finding The Missing Links, 123

students without any exceptional biases (some biases for gender and academic engagement appear to be somewhat inevitable). Its ability to capture a wide range of students ensures that even the most troubled, difficult students to study will nonetheless be represented, allowing for greater representation in research. Finally, it is sensitive to selection and influence effects, as evident from models of changes in academic engagement across the school year and from analyses of the clustering of engagement.

\section{Strengths and Limitations}

This thesis contains several strengths. Due to the small-town nature of the community the data for this study were collected in, the data reflect a representative sampling of this community, as the middle-school the research was originally carried out in served the entire community. Thus, the population of students was not segregated along intellectual, economic, learning, or behavioral differences. In addition, because the data were complete for an entire town, it is known who was not captured, and thus network completeness and saturation could be assessed.

Furthermore, students were not restricted to same-class nominations, but could include any connections they had observed around the school. The inclusion of crossclass ties is important, as social networking studies inside of schools have demonstrated that a large proportion of ties are not entirely contained in the respondent's own classroom: Kindermann (2007) reported that $40 \%$ of nominations were outside of the respondent's classroom, while Gest and colleagues (2007) found that over half of selfreported friends and peers nominated to social groups were from outside of the 
Finding The Missing Links, 124

respondent's own classroom (59.4\% and 51.1\%, respectively), leading the authors to conclude that "the social network is appropriately considered at the level of the entire grade." (p. 11).

Finally, the use of teacher-reported engagement allowed for the analysis of students who were otherwise absent from the data.

However, there are some inherent limitations as well. Although I performed inferential analyses, the results should be generalized with caution. Ultimately, this study is a comparison of networks, and can be thought of as an $\mathrm{N}=1$ study (Snijders, 2011). Great statistical care has been taken, but the most rigorous comparison of the networks would be to create them for different populations and compare afterwards.

Another limitation is that the data I analyzed were collected in the 1990-1991 school year and, thus, are somewhat dated, as they represent a time before the rise of social media and portable electronic communication. On the one hand, this is helpful, as it ensures that students can only "hang out" in person, allowing them to be seen and reported on by others. On the other hand, this may limit the applicability of this study to today's students, where electronic communication may have changed the structure of social networks amongst middle school students. A final limitation is that the use of selfreported groups as a proxy for self-reported friendships may be only moderately valid (Rodkin \& Ahn, 2009). 
Finding The Missing Links, 125

\section{Implications and Future Research}

These findings have several important implications. First, this thesis serves as a strong recommendation for the adoption of collecting social networking information via participant observations and identifying networks using probability scores. Despite the previously demonstrated strengths of the participant observation method compared to self-reports, adoption has been slow, perhaps due to difficulties with implementation (Yugar \& Shapiro, 2001). However, the evidence provided should indicate that any extra trouble may be more than worth the effort.

Second, findings about network properties and analyses of selection and influence processes may help clarify some of the conflicting findings in research on social networks and changes in children's academic engagement. One pattern of findings in particular may be valuable to researchers of students' social environments and their academic engagement: that the probability score method appears to be most responsive to students' engagement.

Third, more efficient measurement of disaffected students' social ties may improve the efficacy of academic interventions, either by increasing awareness of resources (Heinrich, Meyer, \& Whitten, 2010), by the implementation of social network methods in interventions themselves (Kousoulis, Kympouropoulos, Pouli, Economopoulos, \& Vardavas, 2015; Valente et al., 2003), or by improving evaluation of interventions (Valente, Palinkas, Czaja, Chu, \& Brown, 2015).

Finally, this study is, essentially, an evaluation of the validity of social networking methodologies, both in data collection and network identification. The ideal 
Finding The Missing Links, 126

network, the one which is closest to the "actual" social network, will not be the one whose placement of individuals is influenced by personal characteristics (such as gender and academic engagement), unless those personal characteristics are relevant to the structure of the social environment. The findings of this study have demonstrated some potential biases in more commonly used data collection and network identification methodologies (reciprocal nomination methods, commonly employed in friendship research, especially), such as that boys or people with low levels of academic engagement may be underrepresented when reciprocal nominations are used. Consequently, the level of relatedness in a student population may be underestimated, while the level of isolation may be overestimated.

To call the validity of the vast majority of social networking research in grade schools into question based on these findings would be a severe overreach. The structural similarities between the network identification methods, as well as the sometimes small (though significant) biases that were detected suggest that much of the social networking research in the field may only suffer from minimal validity concerns, and that what has been found already would probably be found again even with these results taken into consideration. Instead, I hope that these findings will play a part in the design of stronger studies with a closer eye toward what is and is not being studied (and how things might be different depending on a different methodology).

Ultimately, I feel that the four network identification methods do not represent a continuum of methodology quality, from "bad" to "good", but are instead all more or less "true", but for different types of ties. It is conceivable that the reciprocal nomination 
Finding The Missing Links, 127

network might represent the closest, warmest ties; while the probability score and factoranalytic networks might represent more casual associates; and the rule-based network might represent the loosest, most casual acquaintances. Thus, under this scenario, future researchers would be encouraged to choose the methodology that seems most relevant for them. If they are studying the influence of close friendships, the reciprocal nomination method might be most useful. If they are studying the effects of weak ties, the probability score or rule-based methods might be more applicable (depending on how weak the ties are). For a brief overview of research into different types of ties, see "Types of Relationships" in Appendix B.

In addition, these findings might prove valuable for grade-school teachers. As Kindermann argues, the "invisible hand" of the teacher can influence students in many unexpected ways (2011). One way could be through the manipulation of the students' social environment (intentional or unintentional). Hamm, Farmer, Dadisman, Gravelle, and Murray demonstrated that, when middle-school teachers received training to be more knowledgeable about the peer dynamics within and outside of their classrooms, students benefited from a stronger sense of belonging and an improved attitude toward school (2011). Thus, this thesis can serve as a demonstration of some of the dynamics within social networks, and how those dynamics can appear depending on how the social network is assessed.

Future research could evaluate additional possibilities in network identification, such as what type of tie two students have. One possible advantage of the participant observation method, which is out of the scope of this thesis, is its potential ability to 
Finding The Missing Links, 128

capture multiple types of ties, as students may reciprocally nominate one another as group members, or they may be nominated by others. Do two students who reciprocally nominate one another, and are nominated as being together by many others, influence each other more strongly than two students who do not nominate one another? What about two students who have nominated one another but have not been nominated by third parties? Their tie may represent something of a "secret" relationship, which may carry different qualities than an "open” relationship.

In addition, the theme of validity could be explored further by investigating any differences in the actor-level metrics of students by gender and by engagement levels. In what ways does network identification method affect the structure of boys and girls and students with high and low levels of engagement? Testing my conjecture from above, that each network may simply be measuring a different type of tie, is another possibility. This could be accomplished by determining the properties of networks based on those types of ties from the literature and comparing networks identified using the four methods to those properties.

This thesis also provides several helpful analyses of network similarities and differences, so far lacking in the literature. I have provided a comparison of networks with one another, and not just with self-reported networks (an oft-encountered problem in my review of network comparisons). In addition, I have provided a comparison of networks whose comprehensiveness has not yet been encountered in the literature, determining the extent of overlap and differences in a wide variety of metrics relevant to 
academic engagement. Future research could probe the differences in networks in more depth, by examining other metrics or student characteristics, for example.

This thesis aims to contribute to the ever-developing field of social network analysis a wide-ranging and rigorous comparison of social network gathering and identification methods. I hope to propel future research towards understanding what is gained, lost, and changed through the use of different approaches in social network analysis. Somewhere, beneath all of the individual perceptions, measurement error, and analyses, is the real social environment, waiting to be viewed whole for the aid of students who need it most. 
Finding The Missing Links, 130

\section{References}

Abel, G., Plumridge, L., \& Graham, P. (2002). Peers, networks or relationships:

Strategies for understanding social dynamics as determinants of smoking behaviour. Drugs: Education, Prevention and Policy, 9, 325-338.

ACT. (2011). ACT profile report-national. ACT Inc. Retrieved Oct. $16^{\text {th }} 2011$ from http://www.act.org/newsroom/data/2011/pdf/profile/National2011.pdf

Adams, G. R., \& Montemayor, R. (1983). Identity formation during early adolescence. Journal of Early Adolescence, 3, 193-202.

Adams, R. G., \& Blieszner, R. (1996). Midlife friendship patterns. In N. Vanzetti, \& S. Duck (Eds.), A Lifetime of Relationships (pp. 336-363). Pacific Grove, CA: Brooks.

Adimora, A. A., \& Schoenbach, V. J. (2005). Social context, sexual networks, and racial disparities in rates of sexually transmitted infections. Journal of Infectious Diseases, 191 (Supplement 1), S115-S122.

Aiello, W., Chung, F., \& Lu, L. (2000). A random graph model for massive graphs. Proceedings of the Thirty-Second Annual ACM Symposium on Theory of Computing, 171-180.

Akers, J. F., Jones, R. M., Coyl, D. D. (1998). Adolescent friendship pairs: Similarities in identity status development, behaviors, attitudes, and intentions. Journal of Adolescent Research, 13, 178-201.

Alexanderson, G. (2006). Euler and Königsberg's bridges: A historical view. Bulletin of the American Mathematical Society, 43, 567. 
Finding The Missing Links, 131

Allen, J. P., Porter, M. R., McFarland, F. C., Marsh, P., \& McElhaney, K. B. (2005). The two faces of adolescents' success with peers: Adolescent popularity, social adaptation, and deviant behavior. Child development, 76, 747-760.

Almack, J. C. (1922). The influence of intelligence on the selection of associates. School and Society, 16, 529-530 in Freeman, M. (2004). The Development of Social Network Analysis. Vancouver, British Columbia: Empirical Press.

Altermatt, E. R., \& Pomerantz, E. M. (2003). The development of competence-related and motivational beliefs: An investigation of similarity and influence among friends. Journal of Educational Psychology, 95, 111-123.

Anderson, A. R., Christenson, S. L., Sinclair, M. F., \& Lehr, C. A. (2004). Check \& Connect: The importance of relationships for promoting engagement with school. Journal of School Psychology, 42, 95-113.

Anderson, H. H., \& Anderson, G. L. (1954). Social development. In L. Carmichael (Ed.). Manual of child psychology (2 ${ }^{\text {nd }}$ ed., pp. 1162-1215). New York: Wiley.

Archer, S. L., \& Waterman, A. S. (1983). Identity in early adolescence: A developmental perspective. Journal of Early Adolescence, 3, 203-214.

Aristotle (1934). Rhetoric. Nichomachean ethics. In Aristotle in 23 volumes. Rackman translation. Cambridge: Harvard University Press.

Asher, S. R., \& Hymel, S. (1981). Children's social competence in peer relations: Sociometric and behavior assessment. In J. D. Wine, \& M. D. Snye (Eds.), Social competence (pp. 125-157). New York: Guildford Press. 
Finding The Missing Links, 132

Asher, S. R., Singleton, L. C., Tinsley, B. R., \& Hymel, S. (1979). A reliable sociometric measure for preschool children. Developmental Psychology, 15, 443-444.

Asher, S. R., Singleton, L. C., Tinsley, B. R., \& Hymel, S. (1979). A reliable sociometric measure for preschool children. Developmental Psychology, 15, 443.

Ashmore, R. D., Del Boca, F. K., \& Beebe, M. (2002). “Alkie," "Frat Brother,” and “Jock": Perceived types of college students and stereotypes about drinking. Journal of Applied Social Psychology, 32, 885-907.

Assor, A., Kaplan, H., \& Roth, G. (2002). Choice is good, but relevance is excellent: autonomy-enhancing and suppressing teacher behaviors predicting students' engagement in schoolwork. British Journal of Educational Psychology, 72, 261278.

Aud, S., Hussar, W., Johnson, F., Kena, G., Roth, E., Manning, E., Wang, X., and Zhang, J. (2012). The Condition of Education 2012 (NCES 2012-045). U.S. Department of Education, National Center for Education Statistics. Washington, DC. Retrieved 7/9/2012 from http://nces.ed.gov/pubsearch.

Azmitia, M. (2002). Self, self-esteem, conflicts, and best friendships in early adolescence. In T. M. Brinthaupt, \& R. P. Lipka (Eds.), Understanding early adolescent self and identity: Applications and interventions (pp. 167-192). New York: State University of New York Press.

Badge, J. L., Saunders, N. F., \& Cann, A. J. (2012). Beyond marks: New tools to visualise student engagement via social networks. Research in Learning Technology, 20. 
Finding The Missing Links, 133

Baer, M. (2010). The strength-of-weak-ties perspective on creativity: A comprehensive examination and extension. Journal of Applied Psychology, 95, 592-601.

Bagwell, C. L., Coie, J. D., Terry, R. A., \& Lochman, J. E. (2000). Peer clique participation and social status in preadolescence. Merrill-Palmer Quarterly, 46, $280-305$.

Baines, E., \& Blatchford, P. (2009). Sex differences in the structure and stability of children's playground social networks and their overlap with friendship relations. British Journal of Developmental Psychology, 27, 743-760.

Baker, W. E., \& Faulkner, R. R. (1993). The social organization of conspiracy: Illegal networks in the heavy electrical equipment industry. American Sociological Review, 58, 837-860.

Balland, P. A. (2012). Proximity and the evolution of collaboration networks: evidence from research and development projects within the global navigation satellite system (GNSS) industry. Regional Studies, 46, 741-756.

Bandura, A. (1997). Self-efficacy: The exercise of control. New York: W. H. Freeman. Banks, D., \& Carley, K. (1994). Metric inference for social networks. Journal of Classification, 11, 121-149.

Barber, B. L., Eccles, J. S., \& Stone, M. R. (2001). Whatever happened to the Jock, the Brain, and the Princess? Young adult pathways linked to adolescent activity involvement and social identity. Journal of Adolescent Research, 16(5), 429-455.

Bargh, J. A., \& McKenna, K. Y. (2004). The Internet and social life. Annual Review of Psychology, 55, 573-590. 
Finding The Missing Links, 134

Barkey, K., \& Van Rossem, R. (1997). Networks of Contention: Villages and regional structure in the seventeenth-century Ottoman Empire. American Journal of Sociology, 102, 1345-1382.

Barry, C. M., Madsen, S. D., Nelson, L. J., Carrol, J. S., \& Badger, S. (2009). Friendship and romantic relationship qualities in emerging adulthood: Differential associations with identity development and achieved adulthood criteria. Journal of Adult Development, 16, 209-222.

Batchelder, W. H., Kumbasar, E., \& Boyd, J. P. (1997). Consensus analysis of three-way social network data. Journal of Mathematical Sociology, 22, 29-58.

Bauman, K. E., \& Ennett, S. T. (1996). On the importance of peer influence for adolescent drug use: Commonly neglected considerations. Addiction, 91, 185198.

Beineke, L. W. (1968). Derived graphs of digraphs. in Sachs, H.; Voss, H.-J.; Walter, H.-J., Beiträge zur Graphentheorie, Leipzig: Teubner, 17-33.

Bellman, R. (1958). On a routing problem. Quarterly of Applied Mathematics, 16, 87-90. Benenson, J. F. (1994). Ages four to six years: Changes in the structures of play networks of girls and boys. Merrill Palmer Quarterly, 40, 478-487.

Berkman, L. F., \& Syme, S. L. (1979). Social networks, host resistance, and mortality: A nine-year follow-up study of Alameda County residents. American Journal of Epidemiology, 109, 186-204. 
Bernard, H. R., Killworth, P., \& Sailter, L. (1979). Informant accuracy in social network data IV: a comparison of clique-level structure in behavioral and cognitive network data. Social Networks, 2, 191-218.

Berndt, T. J. (1979). Developmental changes in conformity to peers and parents. Developmental Psychology, 15, 608-616.

Berndt, T. J. (1982). The features and effects of friendship in early adolescence. Child Development, 53, 1447-1460.

Berndt, T. J. (1992). Friendship and friends' influence in adolescence. Current Directions in Psychological Science, 1, 156-159.

Berndt, T. J. (2002). Friendship quality and social development. Current directions in psychological science, 11, 7-10.

Berndt, T. J., \& Keefe, K. (1995). Friends' influence on adolescents' adjustment to school. Child Development, 66, 1312-1329.

Berndt, T. J., Hawkins, J. A., \& Jiao, Z. (1999). Influences of friends and friendships on adjustment to junior high school. Merrill-Palmer Quarterly, 45, 13-41.

Bhattacharyya, S., \& Bickel, P. J. (2013). Subsampling bootstrap of count features of networks. Annals of Statistics. Retrieved from http://arxiv.org/pdf/1312.2645.pdf

Bierman, K. L., \& Wargo, J. (1995). Predicting the longitudinal course associated with aggressive-rejected, aggressive (nonrejected), and rejected (nonaggressive) status. Development and Psychopathology, 7, 669-682. 
Finding The Missing Links, 136

Bierman, K. L., Smoot, D. L., \& Aumiller, K. (1993). Characteristics of aggressiverejected, aggressive (nonrejected), and rejected (nonaggressive) boys. Child Development, 64, 139-151.

Bigelow, B. J. (1977). Children's friendship expectations: A cognitive-developmental study. Child Development, 48, 246-253.

Billy, J. O. G., \& Udry, J. R. (1985). Patterns of adolescent friendship and effects on sexual behavior. Social Psychology Quarterly, 48, 27-41.

Blakemore, S. J., \& Mills, K. L. (2014). Is adolescence a sensitive period for sociocultural processing? Annual review of psychology, 65, 187-207.

Bliss, C. A., Kloumann, I. M., Harris, K. D., Danforth, C. M., \& Dodds, P. S. (2012). Twitter reciprocal reply networks exhibit assortativity with respect to happiness. Journal of Computational Science, 3, 388-397.

Boivin, M., Hymel, S., \& Hodges, E. (2001). Toward a process view of peer rejection and harassment. In J. Juvonen, \& S. Graham (Eds.), Peer harassment in school: The plight of the vulnerable and the victimized (pp. 265-289). New York: Guilford Press.

Bolino, M. C., Turnley, W. H., \& Bloodgood, J. M. (2002). Citizenship behavior and the creation of social capital in organizations. Academy of management review, 27, $505-522$.

Bondonio, D. (1998). Predictors of accuracy in perceiving informal social networks. Social Networks, 20, 301-330. 
Finding The Missing Links, 137

Bonser, F. G. (1902). Chums: A study in youthful friendships. Pedagogical Seminary, 9, $221-236$.

Borgatti, S. P. (2005). Centrality and network flow. Social Networks, 27, 55-71.

Borgatti, S., \& Everett, M. (1999). Models of core/periphery structures. Social Networks, $21,375-395$.

Borgatti, S.P., Everett, M.G., \& Freeman, L.C. (2002). Ucinet for Windows: Software for Social Network Analysis. Harvard, MA: Analytic Technologies.

Bott, H. (1928). Observation of play activities in a nursery school. Genetic Psychology Monographs, 4, 44-88.

Breckelmans, M., Wubbels, T., \& Levy, J. (1993). Student performance, attitudes, instructional strategies and teacher-communication style. In T. Wubbels, \& J. Levy (Eds.), Do you know what you look like? Interpersonal relationships in education (pp. 56-63). Washington, DC: The Falmer Press.

Brewer, D. D. (1995). Patterns in the recall of persons in a department of a formal organization. Journal of Quantitative Anthropology, 5, 255-284.

Brewer, D. D. (2000). Forgetting in the recall-based elicitation of personal and social networks. Social Networks, 22, 29-43.

Brewer, D. D., \& Webster, C.M. (1999). Forgetting of friends and its effects on measuring friendship networks. Alcohol and Drug Abuse Institute, University of Washington, 21, 361-373. 
Finding The Missing Links, 138

Brown, B. B. (1990). Peer groups and peer cultures. In S. S. Feldman, \& G. R. Elliott (Eds.), At the threshold: The developing adolescent (pp. 171-196). Cambridge, MA: Harvard University Press.

Brown, B. B. (1999). Measuring the peer environment of American adolescents. In S. L. Friedman, \& T. D. Wachs (Eds.), Measuring the environment across the life span: Emerging methods and concepts (pp. 59-90). Washington, DC: American Psychological Association.

Brown, B. B., \& Lohr, M. J. (1987). Peer-group affiliation and adolescent self-esteem: An integration of ego-identity and symbolic-interaction theories. Journal of Personality and Social Psychology, 52, 47-55.

Brown, B. B., Feiring, C., \& Furman, W. (1999). Missing the love boat: Why researchers have shied away from adolescent romance. In W.Furman, B. B.Brown, \& C.Feiring (Eds.), The development of romantic relationships in adolescence (pp. 1-16). New York: Cambridge University Press.

Brown, B. B., Herman, M., Hamm, J. V., \& Heck, D. J. (2008). Ethnicity and image: Correlates of crowd affiliation among ethnic minority youth. Child Development, 79, 529-546. 
Finding The Missing Links, 139

Brown, B. B., Mory, M. S., \& Kinney, D. (1994). Casting adolescent crowds in a relational perspective: Caricature, channel and context. In R. Montemayor, G. R. Adams, \& T. P.Gullotta (Eds.), Personal relationships during adolescence (pp. 123-167). Thousand Oaks, CA: Sage in Urberg, K. A., Degirmencioglu, S. M., Tolson, J. M., \& Halliday-Scher, K. (2000). Adolescent social crowds: Measurement and relationship to friendships. Journal of Adolescent Research, 15, $427-445$.

Brown, J. J., \& Reingen, P. H. (1987). Social ties and word of mouth referral behavior. Journal of Consumer Research, 14, 350-362.

Buhl, H. M. (2009). My mother: My best friend? Adults' relationships with significant others across the lifespan. Journal of Adult Development, 16, 239-249.

Bukowski, W. M., \& Hoza, B. (1989). Popularity and friendship: Issues in theory, measurement, and outcome. In T. J. Berndt, \& G. W. Ladd (Eds.), Peer relationships in child development (pp. 15-45). New York: John Wiley, \& Sons.

Bukowski, W. M., Newcomb, A. F., \& Hartup, W. W. (1996). Friendship and its significance in childhood and adolescence: Introduction and comment. In W. Bukowski, A. Newcomb, \& W. Hartup (Eds.), The company they keep: Friendship in childhood and adolescence (pp. 1-15). Cambridge, UK: Cambridge Univ. Press.

Burk, W. J., Kerr, M., \& Stattin, H. (2008). The co-evolution of early adolescent friendship networks, school involvement, and delinquent behaviors. Revue Française de Sociologie, 49, 499-522. 
Burkhardt, M. E., \& Brass, D. J. (1990). Changing patterns or patterns of change: The effects of a change in technology on social network structure and power. Administrative Science Quarterly, 35, 104-127.

Burt, R. S. (1980). Innovation as a structural interest: Rethinking the impact of network position on innovation adoption. Social Networks, 2, 327-355.

Burt, R. S. (1987). Social contagion and innovation: Cohesion versus structural equivalence. American Journal of Sociology, 92, 1287-1335.

Burt, R. S. (2000). Decay functions. Social Networks, 22, 1-28.

Cacioppo, J. T., Hawkley, L. C., \& Thisted, R. A. (2010). Perceived social isolation makes me sad: 5-year cross-lagged analyses of loneliness and depressive symptomatology in the Chicago Health, Aging, and Social Relations Study. Psychology and Aging, 25, 453.

Cairns, R. B. (1994). Lifelines and risks: Pathways of youth in our time. Cambridge University Press.

Cairns, R. B., Cairns, B. D., Neckerman, H. J., Gest, S. D., \& Gariépy, J. L. (1988). Social networks and aggressive behavior: Peer support or peer rejection? Developmental Psychology, 24, 815.

Cairns, R. B., Gariépy, J-L., \& Kindermann, T. (1991). Identifying social clusters in natural settings. Unpublished manuscript. University of North Carolina at Chapel Hill. 
Finding The Missing Links, 141

Cairns, R. B., Leung, M. C., Buchanan, L., \& Cairns, B. D. (1995). Friendships and social networks in childhood and adolescence: Fluidity, reliability, and interrelations. Child Development, 66, 1330-1345.

Cairns, R. B., Perrin, J. E., \& Cairns, B. D. (1985). Social structure and social cognition in early adolescence: Affiliative patterns. Journal of Early Adolescence, 5, 339355.

Cairns, R. B., Xie, H., \& Leung, M. C. (1998). The popularity of friendship and the neglect of social networks: Toward a new balance. New Directions for Child and Adolescent Development, 1998, 25-53.

Caldeira, G. A., \& Patterson, S. C. (1987). Political friendship in the legislature. Journal of Politics, 4, 953-975.

Calvó-Armengol, A., Patacchini, E., \& Zenou, Y. (2009). Peer effects and social networks in education. The Review of Economic Studies, 76, 1239-1267.

Campbell, K. E. (1990). Networks past: A 1939 Bloomington neighborhood. Social Forces, 69, 139-155.

Carley, K. M., \& Krackhardt, D. (1996). Cognitive inconsistencies and non-symmetric friendship. Social Networks, 18, 1-27.

Carmichael, L. (Ed.). (1946). Manual of child psychology. New York: Wiley. Carmichael, L. (Ed.). (1954). Manual of child psychology (2nd ed.). New York: Wiley. Carpenter, D. P., Esterling, K. M., \& Lazer, D. M. (1998). The strength of weak ties in lobbying networks: Evidence from health-care politics in the United States. Journal of Theoretical Politics, 10, 417-444. 
Finding The Missing Links, 142

Carter, R. S., \& Wojtkiewicz, R. A. (2000). Parental involvement with adolescents' education: Do daughters or sons get more help? Adolescence, 35(137), 29-44.

Carver, K., Joyner, K., \& Udry, J. R. (2003). National estimates of adolescent romantic relationships. In P. Florsheim (Ed.), Adolescent romantic relationships and sexual behavior: Theory, research, and practical implications (pp. 291-329). New York: Cambridge University.

Casciaro, T., Carley, K. M., \& Krackhardt, D. (1999). Positive affectivity and accuracy in social network perception. Motivation and Emotion, 23, 285-306.

Centola, D., \& Macy, M. (2007). Complex contagions and the weakness of long ties. American Journal of Sociology, 113, 702-734.

Chen, J. (2005). Relation of academic support from parents, teachers, and peers to Hong Kong adolescents' academic achievement: The mediating role of academic engagement. Genetic, Social, and General Psychology Monographs, 131, 77-127.

Chen, X., Chang, L., \& He, Y. (2003). The peer group as a context: Mediating and moderating effects on relations between academic achievement and social functioning in Chinese children. Child Development, 74, 710-727.

Chen, X., Rubin, K. H., \& Li, D. (1997). Relation between academic achievement and social adjustment: Evidence from Chinese children. Developmental psychology, 33,518 .

Cheung, Y. W. (1997). Family, school, peer, and media predictors of adolescent deviant behavior in Hong Kong. Journal of Youth and Adolescence, 26, 569-596. 
Finding The Missing Links, 143

Cho, H., \& Lee, J. S. (2008). Collaborative information seeking in intercultural computer-mediated communication groups: Testing the influence of social context using social network analysis. Communication Research, 35, 548-573.

Choi, S. Y. (2000). The occupational attainments of the five major dialect groups in Hong Kong. Shatin, New Territories, Hong Kong: The Chinese University of Hong Kong, Hong Kong Institute of Asia-Pacific Studies.

Christakis, N. A., \& Fowler, J. H. (2008). The collective dynamics of smoking in a large social network. New England Journal of Medicine, 358, 2249-2258.

Christakis, N. A., \& Fowler, J. H. (2009). Connected: The surprising power of our social networks and how they shape our lives. Little Brown and Co.: New York.

Christakis, N. A., \& Fowler, J. H. (2013). Social contagion theory: Examining dynamic social networks and human behavior. Statistics in Medicine, 32, 556-577.

Christakis, N., \& Fowler, J. (2007). The spread of obesity in a large social network over 32 years. The New England Journal of Medicine, 9, 357-370.

Cillessen, A., van Ijzendoom, H., van Lieshout, C., \& Hartup, W. (1992). Heterogeneity among peer rejected boys: Subtypes and stabilities. Child Development, 63, 893905.

Clark, M., \& Drewry, D. (1985). Similarity and reciprocity in the friendships of elementary school children. Child Study Journal, 15, 251-264.

Cobb, J. A. (1972). Relationship of discrete classroom behaviors to fourth-grade academic achievement. Journal of Educational Psychology, 63, 74-80.

Cohen, J. (1977). Statistical power analysis for the behavioral sciences (rev. ed.). 
Finding The Missing Links, 144

Cohen-Cole, E., \& Fletcher, J. M. (2008). Detecting implausible social network effects in acne, height, and headaches: longitudinal analysis. BMJ: British Medical Journal, 337, 28-31.

Coie, J. D., Dodge, K. A., \& Kupersmidt, J. B. (1990). Peer group behavior and social status. In S. R. Asher, \& J. D. Coie (Eds.), Peer rejection in childhood (pp. 1759). New York: Cambridge University Press in Doll, B., Spies, R. A., LeClair, C. M., Kurien, S. A., \& Foley, B. P. (2010). Student perceptions of classroom learning environments: Development of the ClassMaps Survey. School Psychology Review, 39, 203-218.

Coie, J., \& Kupersmidt, J. (1983). A behavioral analysis of emerging social status in boys groups. Child Development, 54, 1400-1416.

Coie, J., Dodge, K., \& Kupersmidt, J. (1990). Peer group behavior and social status. In S. Asher, \& J. Coie (Eds.), Peer rejection in childhood (pp. 17-59). Cambridge, UK: Cambridge Univ. Press.

Coie, J., Dodge, K., Terry, R., \& Wright, V. (1991). The role of aggression in peer relations: An analysis of aggression episodes in boys' play groups. Child Development, 62, 812-826.

Coleman, J. S. (1958). Relational analysis: The study of social organizations with survey methods. Human Organization, 17, 28-36. 
Finding The Missing Links, 145

Collins W. A., Van Dulmen, M. (2006). "The course of true love(s)...”: Origins and pathways in the development of romantic relationships. In Romance and Sex in Adolescence and Emerging Adulthood: Risks and Opportunities, ed. A. Booth, A. Crouter, pp. 63-86.

Collins, W. A. (2003). More than myth: The developmental significance of romantic relationships during adolescence. Journal of Research on Adolescence, 13, 1-24.

Collins, W. A., \& Laursen, B. (2004). Parent-adolescent relationships and influence. In R. Lerner, \& L. Steinberg (Eds.), Handbook of adolescent psychology (pp. 331362). New York: Wiley.

Connolly, J. A. (1983). A review of sociometric procedures in the assessment of social competences in children. Applied Research in Mental Retardation, 4, 315-327.

Connolly, J. A., \& Konarski, R. (1994). Peer self-concept in adolescence: Analysis of factor structure and of associations with peer experience. Journal of Research on Adolescence, 4, 385-403.

Connolly, J. A., Craig, W., Goldberg, A., \& Pepler, D. (2004). Mixed-gender groups, dating, and romantic relationships in early adolescence. Journal of Research in Adolescence, 14, 185-207.

Connolly, J. A., Furman, W., \& Konarski, R. (2000). The role of peers in the emergence of heterosexual romantic relationships in adolescence. Child Development, 71, 1395-1408. 
Finding The Missing Links, 146

Connolly, J., Craig, W., Goldberg, A., \& Pepler, D. (1999). Conceptions of cross-sex friendships and romantic relationships in early adolescence. Journal of Youth and Adolescence, 28, 481-494.

Connolly, J., Furman, W., \& Konarski, R. (2000). The role of peers in the emergence of heterosexual romantic relationships in adolescence. Child development, 71, 13951408.

Constant, D., Sproull, L., \& Kiesler, S. (1996). The kindness of strangers: The usefulness of electronic weak ties for technical advice. Organization science, 7, 119-135.

Cook, W. L., \& Kenny, D. A. (2005). The actor-partner interdependence model: A model of bidirectional effects in developmental studies. International Journal of Behavioral Development, 29, 101-109.

Cooper, C. R., \& Grotevant, H. D. (1987). Gender issues in the interface of family experience and adolescents' friendship and dating identity. Journal of Youth and Adolescence, 16, 247-264.

Corsetti, G., Pericoli, M., \& Sbracia, M. (2010). Correlation analysis of financial contagion. In Financial Contagion: The Viral Threat to the Wealth of Nations. New York: Wiley.

Costenbader, E., \& Valente, T. W. (2003). The stability of centrality measures when networks are sampled. Social Networks, 25, 283-307.

Csardi, G., \& Nepusz, T. (2006). The igraph software package for complex network research. InterJournal, Complex Systems, 1695. 
Finding The Missing Links, 147

Curran-Everett, D. (2000). Multiple comparisons: Philosophies and illustrations. American Journal of Physiology-Regulatory, Integrative and Comparative Physiology, 279, R1-R8.

Damon, W. (1992). Teaching as a moral craft and developmental expedition. In F. K. Oser, A. Dick, \& J. Patry (Eds.), Effective and responsible teaching: The new synthesis (pp. 139-153). San Francisco: Jossey-Bass in Doll, B., Spies, R. A., LeClair, C. M., Kurien, S. A., \& Foley, B. P. (2010). Student perceptions of classroom learning environments: Development of the ClassMaps Survey. School Psychology Review, 39, 203-218.

Daudin, J. J., Picard, F., \& Robin, S. (2008). A mixture model for random graphs. Statistics and Computing, 18, 173-183.

Davies, M., \& Kandel, D. B. (1981). Parental and peer influences on adolescents' educational plans: Some further evidence. American Journal of Sociology, 87, $363-387$.

Day, K. M., Hamm, J. V., Lambert, K., \& Farmer, T. W. (2013). Social integration and students' perceptions of the transition to middle school. Journal of Educational and Developmental Psychology, 4, p10.

Denham, S. A. (2006). Social-emotional competence as support for school readiness: What is it and how do we assess it? Early Education and Development, 17, 5789.

Dilorio, C., Kelley, M., \& Hockenberry-Eaton, M. (1999). Communication about sexual issues: Mothers, fathers, and friends. Journal of Adolescent Health, 24, 181-189. 
Finding The Missing Links, 148

Dijkstra, E. W. (1959). A note on two problems in connexion with graphs. Numerische Mathematik, 1, 269-271.

Dion, K. K., \& Berscheid, E. (1974). Physical attractiveness and peer perception among children. Sociometry, 1-12.

DiPietro, J. A. (1981). Rough and tumble play: A function of gender. Developmental Psychology, 17, 50-58;

Dishion, T. J., Andrews, D. W., \& Crosby, L. (1995a). Antisocial boys and their friends in early adolescence: Relationship characteristics, quality, and interactional process. Child Development, 66, 139-151.

Dishion, T. J., Capaldi, D., Spracklen, K. M., \& Li, F. (1995b). Peer ecology of male adolescent drug use. Development and Psychopathology, 7, 803-824.

Dodge, K. (1983). Behavioral antecedents of social status. Child Development, 54, 13861399.

Dolan, L. (1993). The prediction of reading achievement and self-esteem from an index of home educational environment: a study of urban elementary students. Measurement and Evaluation in Guidance, 16, 86-94.

Doll, B. (1996). Children without friends: Implications for practice and policy. School Psychology Review, 25, 165-183.

Doll, B., Spies, R. A., LeClair, C. M., Kurien, S. A., \& Foley, B. P. (2010). Student perceptions of classroom learning environments: Development of the ClassMaps Survey. School Psychology Review, 39, 203-218. 
Finding The Missing Links, 149

Doreian, P. (1989). Network autocorrelation models: Problems and prospects. In D. Griffith (Ed), Spatial Statistics: Past, Present, Future, (pp. 369-389). Ann Arbor: Michigan Document Services.

Doreian, P., \& Stokman, F. N. (1997). The dynamics and evolution of social networks: Processes and principles. In P. Doreian, \& F. N. Stokman (Eds), Evolution of Social Networks (pp. 1-17). New York: Gordon and Breach.

Duffy, A. L., \& Nesdale, D. (2009). Peer groups, social identity, and children's bullying behavior. Social Development, 18, 121-139.

Duijn, M. A., Snijders, T. A., \& Zijlstra, B. J. (2004). p2: A random effects model with covariates for directed graphs. Statistica Neerlandica, 58, 234-254.

Dunphy, D. C. (1963). The social structure of urban adolescent peer groups. Sociometry, 26, 230-246.

Durkheim, E. (1893/1964). The Division of Labor in Society. New York: Free Press in Freeman, M. (2004). The Development of Social Network Analysis. Vancouver, British Columbia: Empirical Press.

Duval, R. (1993). Bootstrapping: A nonparametric approach to statistical inference. Sage.

Eccles, J. S., Lord, S., \& Midgley, C. (1991). What are we doing to adolescents? The impact of educational contexts on early adolescence. American Journal of Education, 99, 521-542.

Eccles, J. S., Midgley, C., Wigfield, A., Buchanan, C. M., Reuman, D., Flanagan, C., \& Mac Iver, D. (1993). Development during adolescence: The impact of stage- 
Finding The Missing Links, 150

environment fit on young adolescents' experiences in schools and in families. American Psychologist, 48, 90-101.

Eccles, J. S., Wigfield, A., Midgley, C., Reuman, D., MacIver, D., \& Feldlaufer, H. (1993). Negative effects of traditional middle schools on students' motivation. Elementary School Journal, 93, 553-574.

Eisenberg, N., Tryon, K., \& Cameron, E. (1984). The relation of preschoolers' peer interaction to their sex-typed toy choices. Child Development, 55, 1044-1050.

Ellis, W. E., \& Zarbatany, L. (2007). Peer group status as a moderator of group influence on children's deviant, aggressive, and prosocial behavior. Child Development, 78, $1240-1254$.

Elmore, R. F., \& Associates. (1990). Restructuring schools: The next generation of educational reform. San Francisco: Jossey-Bass.

Emerson, R. M. (1976). Social exchange theory. Annual review of sociology, 2, 335-362. Emirbayer, M., \& Goodwin, J. (1994). Network analysis, culture, and the problem of agency. American Journal of Sociology, 99, 1411-1454.

Ennett, S. T., \& Bauman, K. E. (1993). Peer group structure and adolescent cigarette smoking: A social network analysis. Journal of Health and Social Behavior, 34 , $226-236$.

Ennett, S. T., \& Bauman, K. E. (1994). The contribution of influence and selection to adolescent peer group homogeneity: The case of adolescent cigarette smoking. Journal of Personality and Social Psychology, 67, 653-663. 
Finding The Missing Links, 151

Ennett, S. T., Bauman, K. E., Hussong, A., Faris, R., Foshee, V. A., Cai, L., et al., (2006). The peer context of adolescent substance use: Findings from social network analysis. Journal of Research on Adolescence, 16, 159-186.

Erdley, C. A., Nangle, D. W., \& Gold, J. A. (1998). Operationalizing the construct of friendship among children: A psychometric comparison of sociometric-based definitional methodologies. Social Development, 7, 62-71.

Erdős, P., \& Rényi, A. (1959). On random graphs I. Publ. Math. Debrecen, 6, 290-297.

Erikson, E. H. (1963). Childhood and society (2 ${ }^{\text {nd }}$ ed.). New York: Norton.

Erikson, E. H. (1968). Identity: Youth and crises. New York: Norton.

Espelage, D. L., Holt, M. K., \& Henkel, R. R. (2003). Examination of peer group contextual effects on aggression during early adolescence. Child Development, $74,205-220$.

Estell, D. B., Cairns, R. B., Farmer, T. W., \& Cairns, B. D. (2002). Aggression in innercity early elementary classrooms: Individual and peer-group configurations. Merrill-Palmer Quarterly, 48, 52-76.

Estell, D. B., Farmer, T. W., \& Cairns, B. D. (2007). Bullies and victims in rural African American youth: Behavioral characteristics and social network placement. Aggressive Behavior, 33, 145-159.

Estell, D. B., Farmer, T. W., Cairns, B. D., \& Clemmer, J. T. (2003). Self-report weapon possession in school and patterns of early adolescent adjustment in rural AfricanAmerican Youth. Journal of Clinical Child and Adolescent Psychology, 32, 442452. 
Finding The Missing Links, 152

Fang, X., Li, X., Stanton, B., \& Dong, Q. (2003). Social network position and smoking experimentation among Chinese adolescents. American Journal of Health Behavior, 27, 257-267.

Farmer, T. W., \& Cairns, B. D. (1991). Social networks and social status in behaviorally disturbed children. Behavioral Disorders, 16, 288-298.

Farmer, T. W., \& Farmer, E. M. Z. (1996). Social relationships of students with exceptionalities in mainstream classrooms: Social networks and homophily. Exceptional Children, 62, 431-450.

Farmer, T. W., \& Rodkin, P. C. (1996). Antisocial and prosocial correlates of classroom social positions: The social network centrality perspective. Social Development, 5 , 174-188.

Farmer, T. W., Estell, D. B., Hall, C. M., Pearl, R., Acker, R. V., \& Rodkin, P. C. (2008). Interpersonal competence configurations, behavior problems, and social adjustment in preadolescence. Journal of Emotional and Behavioral Disorders, $16,195-212$.

Farmer, T. W., Stuart, C. B., Lorch, N. H., \& Fields, E. (1993). The social behavior and peer relations of emotionally and behaviorally disturbed students in residential treatment: A pilot study. Journal of Emotional and Behavioral Disorders, 1, 223234. 
Finding The Missing Links, 153

Farmer, T.W., Estell, D. B., Man-Chi, L., Trott, H., Bishop, J., \& Cairns, B. D. (2003). Individual characteristics, early adolescent peer affiliations, and school dropout: an examination of aggressive and popular group types. Journal of School Psychology, 41, 217-232.

Fawcett, C. A., \& Markson, L. (2010). Similarity predicts liking in 3-year-old children. Journal of experimental child psychology, 105, 345-358.

Fehrmann, P. G., Keith, T. Z., \& Reimers, T. M. (1987). Home influence on school learning: Direct and indirect effects of parental involvement on high school grades. Journal of Educational Research, 80, 330-337.

Feiring, C. (1999). Other-sex friendship networks and the development of romantic relationships in adolescence. Journal of Youth and Adolescence, 28, 495-512.

Feld, S. L. (1981). The focused organization of social ties. American Journal of Sociology, 86, 1015-1035.

Feld, S. L. (1982). Structural determinants of similarity among associates. American Sociological Review, 47, 797-801.

Feld, S. L., \& Elmore, R. (1982). Patterns of sociometric choices: Transitivity reconsidered. Social Psychology Quarterly, 45, 77-85.

Feldman, E., \& Dodge, K. (1987). Social information processing and sociometric status: Sex, age, and situational effects. Journal of Abnormal Child Psychology, 15, 211227.

Finn, J. D. (1993). School Engagement and Students at Risk. Washington, DC: National Center for Educational Statistics, U.S. Department of Education. 
Finding The Missing Links, 154

Fisher, L. A., \& Bauman, K. E. (1988). Influence and selection in the friend-adolescent relationship: Findings from studies of adolescent smoking and drinking. Journal of Applied Social Psychology, 18, 289-314.

Fleiss, J. L. (1971). Measuring nominal scale agreement among many raters. Psychological Bulletin, 76, 378-382.

Fleiss, J. L., Levin, B., \& Paik, M. C. (2003). Statistical methods for rates and proportions. Hoboken, NJ: Wiley.

Flynn, F. J., Reagans, R. E., \& Guillory, L. (2010). Do you two know each other? Transitivity, homophily, and the need for (network) closure. Journal of Personality and Social Psychology, 99, 855-869.

Fournet, J., \& Barrat, A. (2014). Contact patterns among high school students. PloS one, 9, e107878.

Fowler, J. H., \& Christakis, N. A. (2010). Cooperative behavior cascades in human social networks. Proceedings of the National Academy of Sciences, 107, 5334-5338.

Franzese, R. J., Hays, J. C., \& Kachi, A. (2012). Modeling history dependence in network-behavior coevolution. Political Analysis, 20, 175-190.

Fredricks, J. A., Blumenfeld, P. C., \& Paris, A. H. (2004). School Engagement: Potential of the Concept, State of the Evidence. Review of Educational Research, 74, 59109.

Freeman, L. C. (1979). Centrality in networks: I. Conceptual clarification. Social Networks, 1, 215-239. 
Finding The Missing Links, 155

Freeman, M. (1989). "Network representations.” Pp. 11-40 in Research Methods in Social Network Analysis, edited by Linton C. Freeman, Douglas R. White, and A. Kimball Romney. Fairfax, VA: George Mason University.

Freeman, M. (2004). The Development of Social Network Analysis. Vancouver, British Columbia: Empirical Press.

French, D. (1998). Heterogeneity of peer rejected boys: Aggressive and nonaggressive subtypes. Child Development, 59, 976-985.

Furman, W. (1996). The measurement of friendship perceptions: Conceptual and methodological issues. In W. M. Bukowski, A. F. Newcomb, \& W. W. Hartup (Eds.) The company they keep: Friendship in childhood and adolescence (pp. 4165). New York: Cambridge University Press.

Furman, W., \& Buhrmester, D. (1992). Age and sex differences in perceptions of networks of personal relationships. Child Development, 63, 832-837.

Furman, W., \& Shaffer, L. (2003). The role of romantic relationships in adolescent development. In P. Florsheim (Ed.), Adolescent romantic relations and sexual behavior: Theory, research, and practical implications. Mahwah, NJ: Erlbaum.

Furman, W., \& Shomaker, L. B. (2008). Patterns of Interaction in Adolescent Romantic Relationships: Distinct Features and Links to Other Close Relationships. Journal of Adolescence, 31, 771-788. 
Finding The Missing Links, 156

Furman, W., Ho, M. J., \& Low, S. M. (2007). The rocky road of adolescent romantic experience: Dating and adjustment. Friends, lovers and groups: Key relationships in adolescence, eds. R. C. M. E. Engels, M. Kerr and H. Stattin, pp. 61-80, John Wiley, \& Sons, Ltd, West Sussex, England.

Furman, W., Simon, V. A., Shaffer, L., \& Bouchey, H. A. (2002). Adolescents' working models and styles for relationships with parents, friends, and romantic partners. Child development, 73, 241-255.

Gamer, M., Lemon, J., \& Singh, I. F. P. (2012). irr: Various coefficients of interrater reliability agreement. R package version 0.84 .

Gandy, A. (2009). Sequential implementation of Monte Carlo tests with uniformly bounded resampling risk. Journal of the American Statistical Association, 104, $1504-1511$.

Gavin, L. A., \& Furman, W. (1989). Age differences in adolescents' perceptions of their peer groups. Developmental. Psychology, 25, 827-834.

George, T. P., \& Hartmann, D. P. (1996). Friendship networks of unpopular, average, and popular children. Child Development, 67, 2301-2316.

Gershman, E. S., \& Hayes, D S. (1983). Differential stability of reciprocal friendships and unilateral relationships among preschool children. Merrill-Palmer Quarterly, 29, 169-177.

Gershman, E., \& Hayes, D. (1983). Differential stability of reciprocal friendships and unilateral friendships among preschool children. Merrill-Palmer Quarterly, 29, 169-177. 
Finding The Missing Links, 157

Gest, S. D. (2006). Teacher reports of children's friendships and social groups:

Agreement with peer reports and implications for studying peer similarity. Social Development, 15, 248-259.

Gest, S. D., Domitrovich, C. E., \& Welsh, J. A. (2005). Peer academic reputation in elementary school: Associations with changes in self-concept and academic skills. Journal of Educational Psychology, 97, 337-346.

Gest, S. D., Farmer, T. W., Cairns, B. D., \& Xie, H. (2003). Identifying children's peer social networks in school classrooms: Links between peer reports and observed interactions. Social Development, 12, 513-529.

Gest, S. D., Graham-Bermann, S. A., \& Hartup, W. W. (2001). Peer experience: Common and unique features of number of friendships, social network centrality, and sociometric status. Social Development, 10, 23-40.

Gest, S. D., Moody, J., Rulison, K. L., (2007). Density or distinction? The roles of data structure and group detection methods in describing adolescent peer groups. Journal of Social Structure, 6, 1-27.

Ghouila-Houri, A. (1962). Caractérisation des graphes non orientés dont on peut orienter les arrêtes de manière à obtenir le graphe d'une relation d'ordre. Les Comptes rendus de l'Académie des sciences, 254, 1370-1371.

Gilbert, E. N. (1959). Random graphs. Annals of Mathematical Statistics, 30, 1141-1144.

Girvan, M., \& Newman, M. E. (2002). Community structure in social and biological networks. Proceedings of the National Academy of Sciences, 99, 7821-7826. 
Finding The Missing Links, 158

Gleason, T. R., \& Hohmann, L. M. (2006). Concepts of real and imaginary friendships in early childhood. Social Development, 15, 128-144.

Gleason, T. R., Sebanc, A. M., \& Hartup, W. W. (2000). Imaginary companions of preschool children. Developmental Psychology, 36, 419.

González, M. C., Herrmann, H. J., Kertész, J., \& Vicsek, T. (2007). Community structure and ethnic preferences in school friendship networks. Physica A: Statistical Mechanics and its Applications, 379, 307-316.

Goodman, L. A. (1961). Snowball Sampling. Annals of Mathematical Statistics, 32, 148170.

Graham, J. A., \& Cohen, R. (1997). Race and sex as factors in children's sociometric ratings and friendship choices. Social Development, 6, 355-372.

Granovetter, M. S. (1973). The strength of weak ties. American Journal of Sociology, 73, 1360-1380.

Green, A. E., \& White, R. (2007). Attachment to pace: Social networks, mobility and prospects of young people. Joseph Roundtree Foundation. York: England. Last retrieved Oct $2^{\text {nd }}, 2011$ on http://www.jrf.org.uk/publications/attachment-placesocial-networks-mobility-and-prospects-young-people.

Greenberg, M. T., Kusche, C. A., Cook, E. T., \& Quamma, J. P. (1995). Promoting emotional competence in school-aged children: The effects of the PATHS curriculum. Development and Psychopathology, 7, 117-136. 
Finding The Missing Links, 159

Greenwood, C. R., Delquadri, J., \& Hall, R. V. (1984). Opportunity to respond and student academic performance. In W. Heward, T. Heron, D. Hill, \& J. TrapPorter (Eds.), Behavior analysis in education (pp. 58-88). Columbus, $\mathrm{OH}$ : Merrill.

Greenwood, C. R., Hops, H., Walker, H. M., Guild, J. J., Stokes, J., Young, K. R., Keleman, K. S., \& Willardson, M. (1979). Standardized classroom behavior management program PASS: Social validation and replication studies in Utah and Oregon. Journal of Applied Behavior Analysis, 12, 255-271.

Greenwood, C. R., Peterson, P., \& Sideridis, G. (1995). Conceptual methodological and technological advances in classroom observational assessment. Diagnostique, 20, 73-100.

Gronlund, N. E. (1959) Sociometry in the classroom. New York: Harper, 1959 in Newcomb, A. F., Bukowski, W. M., \& Pattee, L. (1993). Children's peer relations: A meta-analytic review of popular, rejected, neglected, controversial, and average sociometric status. Psychological Bulletin, 113, 99-128.

Guay, F., Boivin, M., \& Hodges, E. V. (1999). Social comparison processes and academic achievement: The dependence of the development of self-evaluations on friends' performance. Journal of Educational Psychology, 91, 564.

Guay, F., Boivin, M., \& Hodges, E. V. (1999). Predicting change in academic achievement: A model of peer experiences and self-system processes. Journal of Educational Psychology, 91, 105-115. 
Finding The Missing Links, 160

Haberman, S. J. (1988). A warning on the use of chi-squared statistics with frequency tables with small expected cell counts. Journal of the American Statistical Association, 83, 555-560.

Hallinan, M. T. (1980). Patterns of cliquing among youth. Friendship and social relations in children, 321-342.

Hallinan, M. T., \& Williams, R. (1989). Interracial friendship choice in secondary schools. American Sociological Review, 54, 67-78.

Hamm, J. V., Farmer, T. W., Dadisman, K., Gravelle, M., \& Murray, A. R. (2011). Teachers' attunement to students' peer group affiliations as a source of improved student experiences of the school social-affective context following the middle school transition. Journal of Applied Developmental Psychology, 32, 267-277.

Hammer, M. (1980). Social access and the clustering of personal connections. Social Networks, 2, 305-325.

Hammer, M. (1984). Explorations into the meaning of social network interview data. Social Networks, 6, 341-371.

Hampton, K. N., \& Wellman, B. (2000). Examining community in the digital neighborhood: early results from Canada's wired suburb. In T. Ishida, \& K. Isbister (Eds), Digital cities: Technologies, Experiences, and Future Perspectives (pp. 194-208). Heidelberg: Springer-Verlag. 
Finding The Missing Links, 161

Hampton, K. N., \& Wellman, B. (2000). Examining community in the digital neighborhood: early results from Canada's wired suburb. In T. Ishida, K. Isbister (eds), Digital Cities: Technologies, Experiences and Future Perspectives, (pp. 194-208). Springer Berlin Heidelberg.

Hamre, B., \& Pianta, R. (2001). Early teacher-child relationships and the trajectory of children's school outcomes through eighth grade. Child Development, 72, 625638.

Hansson, R. O., \& Carpenter, B. N. (1994). Relationships in old age: Coping with the challenge of transition. New York: Guilford Press.

Harary, F., \& Palmer, E. M. (1973). Graphical Enumeration. New York, NY: Academic Press.

Harrist, A. W., Zaia, A. F., Bates, J. E., Dodge, K. A., \& Pettit, G. S. (1997). Subtypes of social withdrawal in early childhood: Sociometric status and social-cognitive differences across four years. Child Development, 68, 278-294.

Hart, P. E., Nilsson, N. J., \& Raphael, B. (1968). A formal basis for the heuristic determination of minimum cost paths. IEEE Transactions on Systems Science and Cybernetics SSC4, 2, 100-107.

Harter, S. (1999). The construction of the self. New York: Guilford Press.

Hartup, W. (1983). Peer relations. In P. Mussen (Series Ed.), \& E. M. Hetherington (Volume Ed.), Handbook of child psychology: Vol. 4. Socialization, personality, and social development (4th ed.) (pp. 103-198). New York: Wiley. 
Finding The Missing Links, 162

Hartup, W. W. (1970). Peer interaction and social organization. In P. Mussen (Ed.), Carmichael's manual of child psychology (pp. 361-455). NY: Wiley.

Hartup, W. W. (1992). Friendships and their developmental significance. In H. McGarle (Ed.), Childhood Social Development: Contemporary Perspectives (pp. 175-205). Hillsdale, NJ: Lawrence Erlbaum.

Hartup, W. W. (1996). The company they keep: Friendships and their developmental significance. Child Development, 67, 1-13.

Hartup, W. W., Laursen, B., Stewart, M. I., \& Eastenson, A. (1988). Conflict and the friendship relations of young children. Child Development, 1590-1600.

Hatzichristou, C., \& Hopf, D. (1996). A multiperspective comparison of peer sociometric status groups in childhood and adolescence. Child Development, 67, 1085-1102.

Hayes, D. S. (1978). Cognitive bases for liking and disliking among preschool children. Child Development, 49, 906-909.

Hayes, D., Gershman, E., \& Bolin, L. (1980). Friends and enemies: Cognitive bases for preschool children's unilateral and reciprocal relationships. Child Development, $51,1276-1279$.

Hayvren, M., \& Hymel, S. (1984). Ethical issues in sociometric testing: Impact of sociometric measures on interaction behavior. Developmental Psychology, 20, 844-849.

He, Y. (2007). Confucius and Aristotle on friendship: A comparative study. Frontiers of Philosophy in China, 2, 291-307. 
Finding The Missing Links, 163

Heckman, J. J. (2007). The economics, technology, and neuroscience of human capability formation. Proceedings of the National Academy of Sciences, 104, 13250-13255.

Heinrich, C. J., Meyer, R. H., \& Whitten, G. (2010). Supplemental education services under No Child Left Behind: Who signs up, and what do they gain? Educational Evaluation and Policy Analysis, 32, 273-298.

Held, M., \& Karp, R. M. (1962). A dynamic programming approach to sequencing problems. Journal of the Society for Industrial and Applied Mathematics, 10, 196-210.

Henry, D. B., \& Kobus, K. (2007). Early adolescent social networks and substance use. The Journal of Early Adolescence, 27, 346-362

Hill, H. M., Levermore, M., Twaite, J., \& Jones, L. P. (1996). Exposure to community violence and social support as predictors of anxiety and social emotional behavior among African American children. Journal of Child and Family Studies, 5, 399414.

Hintze, J. M., Stoner, G., \& Bull, M. H. (2000). Analogue assessment: Research and practice in evaluating emotional and behavioral problems. In E. S. Shapiro, \& T. R. Kratochwill (Eds.), Behavioral assessment in schools: Theory, research, and clinical foundations (2nd ed.) (pp. 104-138). New York: The Guilford Press.

Hinz, O., Skiera, B., Barrot, C., \& Becker, J. U. (2011). Seeding strategies for viral marketing: An empirical comparison. Journal of Marketing, 75, 55-71. 
Finding The Missing Links, 164

Hlebec, V. (1993). Recall versus recognition, a comparison of the two alternative procedures for collecting social network data. In: Ferligoj, A., Kramberger, A. (Eds.), Developments in Statistics and Methodology. Proceedings of the International Conference on Methodology and Statistics, Bled, Slovenia, University of Ljubljana, Ljubljana, Slovenia, September, 1992 in Brewer, D. D. (2000). Forgetting in the recall-based elicitation of personal and social networks. Social Networks, 22, 29-43.

Hlebec, V., \& Ferligoj, A. (2001). Respondent mood and the instability of survey network measurements. Social Networks, 23, 125-139.

Hobson, J. A. (1884/1954). The Evolution of Modern Capitalism; A Study of Machine Production. London, New York: Allen, \& Unwin, Macmillan.

Hodges, E. V., Boivin, M., Vitaro, F., \& Bukowski, W. M. (1999). The power of friendship: protection against an escalating cycle of peer victimization. Developmental psychology, 35, 94.

Hoff, P. D., Raftery, A. E., \& Handcock, M. S. (2002). Latent space approaches to social network analysis. Journal of the American Statistical Association, 97, 1090-1098.

Hogg, T., Wilkinson, D. M., Szabo, G., \& Brzozowski, M. J. (2008). Multiple relationship types in online communities and social networks. American Association for Artificial Intelligence-Social Information Processing (AAAI Press, Palo Alto, CA). 
Finding The Missing Links, 165

Holland, P. W., \& Leinhardt, S. (1981). An exponential family of probability distributions for directed graphs. Journal of the American Statistical Association, 76, 33-50.

Holland, P. W., Laskey, K. B., \& Leinhardt, S. (1983). Stochastic blockmodels: First steps. Social Networks, 5, 109-137.

Hops, H., \& Cobb, J. A. (1973). Survival behaviors in the educational setting: Their implications for research and intervention. In L. A. Hamerlynck, L. C. Handy, \& E. J. Mash (Eds.), Behavior change: Methodology, concepts and practice (pp. 193-208). Champaign, IL: Research Press.

Howes, C. (1988). Same-and cross-sex friendships: Implications for interaction and social skills. Early Childhood Research Quarterly, 3, 21-37.

Howes, C. (1996). The earliest friendships. In W. Bukowski, A. Newcomb, \& W. Hartup (Eds.), The company they keep: Friendship in childhood and adolescence (pp. 6686). Cambridge, UK: Cambridge Univ. Press.

Howes, C., \& Phillipsen, L. (1992). Gender and friendship: Relationships within peer groups of young children. Social Development, 1, 230-242.

Howes, C., Unger, O. A., \& Matheson, C. (1991). Social pretend play in toddlers: Parallels with social play and with solitary pretend. Child Development, 60, 7784.

Hubbard, R. M. (1929). A method of studying spontaneous group formation. In D. S. Thomas (ed), Some New Techniques for Studying Social Behavior, (pp. 76-85). Child Development Monograms. 
Finding The Missing Links, 166

Huber, F., \& Bonnet, C. (1792). Nouvelles observations sur les abeilles: adressâees áa M. Charles Bonnet. Geneve: Barde Manget. in Freeman, M. (2004). The Development of Social Network Analysis. Vancouver, British Columbia: Empirical Press.

Hubert, L. J., \& Schultz, J. (1976). Quadratic assignment as a general data analysis strategy. British Journal of Mathematical and Statistical Psychology, 29, 190241.

Hughes, J., \& Kwok, O. (2007). Influence of student-teacher and parent-teacher relationships on lower achieving readers' engagement and achievement in the primary grades. Journal of Educational Psychology, 99, 39-51.

Hundley, R. J., \& Cohen, R. (1999). Children's relationships with classmates: A comprehensive analysis of friendship nominations and liking. Child Study Journal, 29, 233-246.

Hundley, R. J., \& Cohen, R. (1999). Children's Relationships with Classmates: A Comprehensive Analysis of Friendship Nominations and Liking. Child Study Journal, 29, 233-46.

Hunt, R. J. (1986). Percent agreement, Pearson's correlation, and kappa as measures of inter-examiner reliability. Journal of Dental Research, 65, 128-130.

Hyman, S. M., Gold, S. N., \& Cott, M. A. (2003). Forms of social support that moderate PTSD in childhood sexual abuse survivors. Journal of Family Violence, 18, 295300. 
Finding The Missing Links, 167

Ibarra, H. (1992). Homophily and differential returns: Sex differences in network structure and access in an advertising firm. Administrative Science Quarterly, 37, 422-447.

Ibarra, H. (1995). Race, opportunity, and diversity of social circles in managerial networks. Academy of Management Journal, 38, 673-703.

Iverson, A. M., \& Iverson, G. L. (1996). Children's long-term reactions to participating in sociometric assessment. Psychology in the Schools, 33, 103-112.

Iyengar, R., Van den Bulte, C., \& Valente, T. W. (2011). Opinion leadership and social contagion in new product diffusion. Marketing Science, 30, 195-212.

Jenssen, J. J., \& Greve, A. (2002). Does the degree of redundancy in social networks influence the success of business start-ups? International Journal of Entrepreneurial Behaviour, \& Research, 8, 254-267.

Jiang, X. L., \& Cillessen, A. H. N. (2005). Stability of continuous measures of sociometric status: a meta-analysis. Developmental Review, 25, 1-25.

Johnson, D. W., Johnson, R. T., Buckman, L. A., \& Richards, P. S. (1998). The effect of prolonged implementation of cooperative learning on social support within the classroom. The Journal of Psychology, 119, 405-411.

Johnson, J. C., \& Orbach, M. K. (2002). Perceiving the political landscape: ego biases in cognitive political networks. Social Networks, 24, 291-310.

Johnson, M. P., \& Leslie, L. (1982). Couple involvement and network structure: A test of the dyadic withdrawal hypothesis. Social Psychology Quarterly, 45, 34-43. 
Finding The Missing Links, 168

Jones, R. M., Vaterlaus, J. M., Jackson, M. A., \& Morrill, T. B. (2014). Friendship characteristics, psychosocial development, and adolescent identity formation. Personal Relationships, 21, 51-67.

Josselson, R. (1992). The space between us: Exploring the dimensions of human relationships. San Francisco: Jossey-Bass.

Josselson, R. (1996). Revising herself: The story of women's identity from college to midlife. New York: Oxford University Press.

Joyner, K., \& Udry, J. R. (2000). You don't bring me anything but down: Adolescent romance and depression. Journal of Health and Social Behavior, 41, 369-391.

Junco, R. (2012). The relationship between frequency of Facebook use, participation in Facebook activities, and student engagement. Computers, \& Education, 58, 162171.

Junco, R., Heiberger, G., Loken, E. (2011). The effect of Twitter on college student engagement and grades. Journal of Computer Assisted Learning, 27, 119-132.

Juvonen, J. (2007). Reforming middle schools: Focus on continuity, social connectedness, and engagement. Educational Psychologist, 42, 197-208.

Kalish, Y., \& Robins, G. (2006). Psychological predispositions and network structure: The relationship between individual predispositions, structural holes and network closure. Social Networks, 28, 56-84.

Kalmijn, M. (1998). Intermarriage and homogamy: Causes, patterns and trends. Annual Review of Sociology, 24, 395-421. 
Finding The Missing Links, 169

Kandel, D. B. (1978). Homophily, selection, and socialization in adolescent friendship pairs. American Journal of Sociology, 48, 427-436.

Keating, N. L., O'Malley, A. J., Murabito, J. M., Smith, K. P., \& Christakis, N. A. (2011). Minimal social network effects evident in cancer screening behavior. Cancer, 117, 3045-3052.

Kenny, D. A., \& Judd, C. M. (1986). Consequences of violating the independence assumption in analysis of variance. Psychological Bulletin, 99, 422-431.

Kerns, K. (2000). Types of preschool friendships. Personal Relationships, 7, 311-324.

Kerpelman, J. L., \& Pittman, J. F. (2001). The instability of possible selves: Identity processes within late adolescents' close peer relationships. Journal of Adolescence, 24, 491-512.

Kesner, J. E. (2000). Teacher characteristics and the quality of child-teacher relationships. Journal of School Psychology, 28, 133-149.

Keßler, C. (2010). Defining and using types of relationships in social networks. 2010 Specialist Meeting-Spatio-Temporal Constraints on Social Networks, 1-2.

Kiesner, J., Poulin, F., \& Nicotra, E. (2003). Peer relations across contexts: Individualnetwork homophily and network inclusion in and after school. Child Development, 74, 1328-1343.

Kim, C. (2008). Social networks and political participation: How do networks mattter? Social Forces, 87, 961-982. 
Finding The Missing Links, 170

Kindermann, T. A. (1993). Natural peer groups as contexts for individual development:

The case of children's motivation in school. Developmental Psychology, 29, 970977.

Kindermann, T. A. (2007). Effects of naturally existing peer groups on changes in academic engagement in a cohort of sixth graders. Child Development, 78, 11861203.

Kindermann, T. A. (2011). Commentary: The invisible hand of the teacher. Journal of Applied Developmental Psychology, 32, 304-308.

Kindermann, T. A., \& Gest, S. D. (2009). Assessment of the peer group: Identifying social networks in natural settings and measuring their influences. In K. H. Rubin, W. Bukowski, \& B. Laursen (Eds.), Handbook of peer interactions, relationships, and groups (pp. 100-120). New York: Guilford.

Kindermann, T. A., McCollam, T. L., \& Gibson, E. (1996). Peer networks and students’ classroom engagement during childhood and adolescence. In Juvonen, J., \& Wentzel, K. R. (Eds.), Social motivation: understanding children's school adjustment (279-313). Cambridge, England: Cambridge University Press.

Kinzler, K. D., \& Spelke, E. S. (2011). Do infants show social preferences for people differing in race? Cognition, 119, 1-9.

Kinzler, K. D., Shutts, K., DeJesus, J., \& Spelke, E. S. (2009). Accent trumps race in guiding children's social preferences. Social cognition, 27, 623. 
Finding The Missing Links, 171

Kipke, M. D., Montgomery, S. B., Simon, T. R., Unger, J. B., \& Johnson, C. J. (1997). Homeless youth: Drug use patterns and HIV risk profiles according to peer group affiliation. AIDS and Behavior, 1, 247-259.

Kipke, M. D., Unger, J. B., O'Connor, S., Palmer, R. F., \& LaFrance, R. (1997). Street youth, their peer group affiliation and differences according to residential status, subsistence patterns, and use of services. Adolescence, 32, 655-669.

Kirch, P. V. (2010). How chiefs became kings: Divine kingship and the rise of archaic states in ancient Hawai'i. Los Angeles, California: University of California Press.

Kirke, D. M. (2004). Chain reactions in adolescents' cigarette, alcohol, and drug use: Similarity through peer influence or the patterning of ties in peer networks? Social Networks, 26, 3-28.

Klapisch-Zuber, C. (2000). L'Ombre des Ancêtres: Essai sur l'Imaginaire Médiéval de la Parenté. Paris: Fayard in Freeman, M. (2004). The Development of Social Network Analysis. Vancouver, British Columbia: Empirical Press.

Klem, A. M., \& Connell, J. P. (2004). Relationships matter: Linking teacher support to student engagement and achievement. Journal of School Health, 74, 262-273.

Knecht, A., Snijders, T. A., Baerveldt, C., Steglich, C. E., \& Raub, W. (2010). Friendship and delinquency: Selection and influence processes in early adolescence. Social Development, 19, 494-514.

Kogan, S. M., Wejnert, C., Chen, Y.-F., \& Brody, G. H. (2011). Respondent-driven sampling with hard-to-reach emerging adults: An introduction and case study with rural African Americans. Journal of Adolescent Research, 26, 30-60. 
Finding The Missing Links, 172

Kossinets, G., \& Watts, D. J. (2006). Empirical analysis of an evolving social network. Science, 311, 88-90.

Koster, M., Pijl, S. J., Nakken, H., \& Van Houten, E. (2010). Social participation of students with special needs in regular primary education in the Netherlands. International Journal of Disability, Development and Education, 57, 59-75.

Kousoulis, A. A., Kympouropoulos, S. P., Pouli, D. K., Economopoulos, K. P., \& Vardavas, C. I. (2015). From the classroom to Facebook: A fresh approach for youth tobacco prevention. American Journal of Health Promotion. In-press.

Kovacs, D. M., Parker, J. G., \& Hoffman, L. W. (1996). Behavioral, affective, and social correlates of involvement in cross-sex friendship in elementary school. Child Development, 67, 2269-2286.

Kowalski, K. (2003). The emergence of ethnic and racial attitudes in preschool-aged children. The Journal of social psychology, 143, 677-690.

Krackhardt, D. (1987). Cognitive social structures. Social Networks, 9, 109-134.

Krackhardt, D., \& Kilduff, M. (2002). Structure, culture and Simmelian ties in entrepreneurial firms. Social networks, 24, 279-290.

Krappman, L. (1996). Amicitia, drujb, shin-yu, philia, freundschaft, friendship: On the cultural diversity of a human relationship. In W. Bukowski, A. Newcomb, \& W. Hartup (Eds.), The company they keep: Friendships in childhood and adolescence. Cambridge studies in social and emotional development (pp. 19-40). Cambridge, UK: Cambridge Univ. Press. 
Finding The Missing Links, 173

Kruskal, J. B. (1956). On the Shortest Spanning Subtree of a Graph and the Traveling Salesman Problem. In: Proceedings of the American Mathematical Society, 7, 4850.

Kumbasar, E., Romney, A. K., \& Batchelder, W. H. (1994). Systematic biases in social perception. American Journal of Sociology, 100, 477-505.

Kun, Z., Guangyi, Z., Deshun, Z., Jun, Y., \& Jinrong, S. (2014). Link formation in undergraduate students' friendship network. In Behavior, Economic and Social Computing (BESC), 2014 International Conference on (pp. 1-5). IEEE.

Kurdek, L. A., \& Sinclair, R. J. (2000). Psychological, family, and peer predictors of academic outcomes in first through fifth grade children. Journal of Educational Psychology, 92, 449-457.

Kuttler, A. F., La Greca, A. M., \& Prinstein, M. J. (1999). Friendship qualities and social-emotional functioning of adolescents with close, cross-sex friendships. Journal of Research on Adolescence, 9, 339-366.

Kyratzis, A. (2004). Talk and interaction among children and the co-construction of peer groups and peer culture. Annual Review of Anthropology, 33, 625-649.

La Greca, A. M., \& Harrison, H. M. (2005). Adolescent peer relations, friendships, and romantic relationships: Do they predict social anxiety and depression? Journal of Clinical Child and Adolescent Psychology, 34, 49-61.

La Greca, A. M., Prinstein, M. J., \& Fetter, M. D. (2001). Adolescent peer crowd affiliation: Linkages with health-risk behaviors and close friendships. Journal of Pediatric Psychology, 26, 131-143. 
Finding The Missing Links, 174

Ladd, G. W. (1990). Having friends, keeping friends, making friends, and being liked by peers in the classroom: Predictors of children's early school adjustment? Child Development, 61, 1081-1100.

Ladd, G. W., \& Price, J. M. (1987). Predicting children's social and school adjustment following the transition from preschool to kindergarten. Child Development, 58, 1168-1189.

LaFreniere, P., Strayer, F. F., \& Gauthier, R. (1984). The emergence of same-sex preferences among preschool peers: A developmental ethological perspective. Child Development, 55, 1958-1965.

Laghi, F., Baiocco, R., Di Norcia, A., Cannoni, E., Baumgartner, E., \& Bombi, A. S. (2014). Emotion understanding, pictorial representations of friendship and reciprocity in school-aged children. Cognition and Emotion, 28, 1338-1346.

Lamis, D. A., Wilson, C. K., King, N. M., \& Kaslow, N. J. (2014). Child abuse, social support, and social functioning in African American children. Journal of Family Violence, 29, 881-891.

Larson, R., \& Richards, M. H. (1991). Daily companionship in late childhood and early adolescence: Changing developmental contexts. Child Development, 62, 284-300.

Larson, R., \& Richards, M. H. (1994). Divergent realities: The emotional lives of mothers, fathers, and adolescents. New York: Basic Books.

Larson, R., Csikszentmihalyi, M., \& Graef, R. (1980). Mood variability and the psychosocial adjustment of adolescents. Journal of Youth and Adolescence, 9, 469-490. 
Finding The Missing Links, 175

Laumann, E. O., \& Pippi, F. (1976). Networks of Collective Action: A Perspective on Community Influence Systems. New York: academic press.

Laundau, S., \& Milich, R. (1990). Assessment of children's social status and peer relations. In A. LaGreca (Ed.), Through the eyes of a child: Obtaining selfreports from children and adolescents (pp. 259-291). Needham Heights, MA: Allyn, \& Bacon.

Laursen, B. (1995). Conflict and social interaction in adolescent relationships. Journal of Research on Adolescence, 5, 55-70.

Lazarsfeld, P. F., \& Merton, R. K. (1954). Friendship as a social process: A substantive and methodological analysis. In Freedom and Control in Modern Society, pp. 1866, ed. M. Berger, T. Abel, C. Page.

Lazarsfeld, P.F., \& Henry, N. W. (1968). Latent Structure Analysis. Boston: Houghton Mifflin.

LeBon, G. (1897/1995). The Crowd. New Brunswick, N.J.: Transaction Pub in Freeman.

Lemann, T. B., \& Solomon, R. L. (1952). Group characteristics as revealed in sociometric patterns and personality ratings. Sociometry, 15, 7-90.

Leung, M. (1998). A user manual for SCM 4.0. Chapel Hill: University of North Carolina, CDS.

Leung, M.-C. (1996). Social networks and self enhancement in Chinese children: A comparison of self-reports and peer reports of group membership. Social Development, 5, 146-157.

Levesque, R. J. R. (1993). The romantic experience of adolescents in satisfying love relationships. Journal of Youth and Adolescence, 22, 219-251. 
Finding The Missing Links, 176

Levin, D. Z., \& Cross, R. (2004). The strength of weak ties you can trust: The mediating role of trust in effective knowledge transfer. Management science, 50, 14771490.

Lewis, K., Gonzalez, M., \& Kaufman, J. (2012). Social selection and peer influence in an online social network. Proceedings of the National Academy of Sciences, 109, 6872.

Liem, G. A. D., \& Martin, A. J. (2011). Peer relationships and adolescents' academic and non-academic outcomes: Same-sex and opposite-sex peer effects and the mediating role of school engagement. British Journal of Educational Psychology, $81,183-206$.

Lin, N. (1999). Social networks and status attainment. Annual Review of Sociology, 25, 467-487.

Lind, P. G., da Silva, L. R., Andrade Jr., J. S., \& Herrmann, H. J. (2007). Spreading gossip in social networks. Physical Review E, 76.

Lipsitz, J., Jackson, A. W., \& Austin, L. M. (1997). What works in middle-grades school reform. Phi Delta Kappan, 78, 517.

Lishner, D. A., Cooter, A. B., \& Zald, D. H. (2008). Rapid emotional contagion and expressive congruence under strong conditions. Journal of Nonverbal Behavior, $32,225-239$.

Louch, H. (2000). Personal network integration: Transitivity and homophily in strong-tie relations. Social Networks, 22, 45-64. 
Finding The Missing Links, 177

Luster, T., \& McAdoo, H. P. (1994). Factors related to the achievement and adjustment of young African American children. Child Development, 65, 1080-1094.

Luther, S., \& McMahon, T. (1996). Peer reputation among inner-city adolescents: Structure and correlates. Journal of Research on Adolescence, 6, 581-603.

Ma, H. K., Shek, D. T. L., Cheung, P. C., \& Lam, C. O. B. (2000). Parental, peer, and teacher influences on the social behavior of Hong Kong Chinese adolescents. The Journal of Genetic Psychology, 161, 65-78.

Ma, H. K., Shek, D. T. L., Cheung, P. C., \& Lee, R. Y. P. (1996). The relation of prosocial and antisocial behavior to personality and peer relationships of Hong Kong Chinese adolescents. The Journal of Genetic Psychology, 157, 255-266.

Määttä, S., Stattin, H., \& Nurmi, J-E (2006). Achievement strategies in peer groups and adolescents' school adjustment and norm-breaking behavior. Scandinavian Journal of Psychology, 47, 273-280.

Maccoby, E. E. (1990). Gender and relationships. American Psychologist, 45, 513-520.

Maccoby, E. E., \& Jacklin, C. N. (1987). Gender segregation in childhood. Advances in Child Development and Behavior, 20, 239-287.

Malecki, C. K., \& Elliott, S. N. (2002). Children's social behaviors as predictors of academia achievement: a longitudinal analysis. School Psychology Quarterly, 17, $1-23$.

Mantel, N. (1967). The detection of disease clustering and a generalized regression approach. Cancer Research, 27, 209-220. 
Finding The Missing Links, 178

Marcia, J. E. (1966). Development and validation of ego-identity status. Journal of Personality and Social Psychology, 3, 551-558.

Markowitz, F. E., Bellair, P. E., Liska, A. E., \& Liu, J. (2001). Extending social disorganization theory: modeling the relationships between cohesion, disorder, and fear. Criminology, 39, 293-319.

Marks, H. M. (2000). Student engagement in instructional activity: Patterns in the elementary, middle, and high school years. American Educational Research Journal, 37, 153-184.

Marsden, P. V. (1990). Network data and measurement. Annual Review of Sociology, 16, 435-463.

Martin, C. L., Fabes, R. A., Evans, S. M., \& Wyman, H. (1999). Social cognition on the playground: Children's beliefs about playing with girls versus boys and their relations to sex segregated play. Journal of Social and Personal Relationships, 16, 751-771.

Martin, M., Fergus, E., \& Noguera, P. (2010). The academic characteristics of Black and Latino boys that matter in achievement: an exploratory achievement model of boys in single--sex schools. Metropolitan Center for Urban Education.

Martineau, H. (1835/2000). The Positive Philosophy of Auguste Comte. Kitchner, Ontario: Batoche in Freeman, M. (2004). The Development of Social Network Analysis. Vancouver, British Columbia: Empirical Press.

Maryanski, A. R. (1987). African ape social structure: Is there strength in weak ties? Social Networks, 9, 191-215. 
Finding The Missing Links, 179

Masten, A. S., Coatsworth, J. D., Neemann, J. 1., Gest, S. D., Tellegen, A., \& Garmezy, N. (1995). The structure and coherence of competence from childhood through adolescence. Child Development, 66, 1635-1659.

Masten, A. S., Hubbard, J. J., Gest, S. D., Tellegen, A., Garmezy, N., \& Ramirez, M. (1999). Competence in the context of adversity: Pathways to resilience and maladaptation from childhood to late adolescence. Development and Psychopathology, 11, 143-169.

Mayhew, B. H., McPherson, M., Rotolo, T., \& Smith-Lovin, L. (1995). Sex and ethnic heterogeneity in face-to-face groups in public places: an ecological perspective on social interaction. Social Forces, 74, 15-52.

McAdams, D. P. (1993). The stories we live by: Personal myths and the making of the self. New York: Morrow.

McAdams, D. P. (2001). The psychology of life stories. Review of General Psychology, $5,100-122$.

McConnell, S. R., \& Odom, S. L. (1986). Sociometrics: Peer-referenced measures and the assessment of social competence. In P. Strain, M. Guralnick, \& H. M. Walker (Eds.), Children's social behavior: Development, assessment, and modification (pp. 215-286). New York: Academic Press.

McDermott, R., Fowler, J. H., \& Christakis, N. A. (2013). Breaking up is hard to do, unless everyone else is doing it too: Social network effects on divorce in a longitudinal sample. Social Forces, sot096. 
Finding The Missing Links, 180

McFarland, D. A., Moody, J., Diehl, D., Smith, J. A., \& Thomas, R. J. (2014). Network ecology and adolescent social structure. American Sociological Review, 79, 10881121.

McLean, K. C. (2005). Late adolescent identity development: narrative meaning making and memory telling. Developmental psychology, 41, 683.

McPherson, M., Smith-Lovin, L., \& Cook, J. M. (2001). Birds of a Feather: Homophily in Social Networks. Annual Review of Sociology, 27, 415-444.

Mednick, S. C., Christakis, N. A., \& Fowler, J. H. (2010). The spread of sleep loss influences drug use in adolescent social networks. PloS one, 5, e9775.

Merrill, K. W. (1999). Behavioral, social, and emotional assessment of children. Mahwah, NJ: Lawrence Erlbaum Associates.

Miller, K. E., Farrell, M. P., Barnes, G. M., Melnick, M. J., \& Sabo, D. (2005). Gender/Racial differences in jock identity, dating, and adolescent sexual risk. Journal of Youth and Adolescence, 34, 123-136.

Miller, T. W. (Ed.). (2010). Handbook of stressful transitions across the lifespan (p. xxiii). Springer.

Moller, L. C., \& Serbin, L. A. (1996). Antecedents of toddler gender segregation: Cognitive consonance, gender-typed toy preferences and behavioral compatibility. Sex Roles, 35, 445-460.

Molloy, L. E., Gest, S. D., \& Rulison, K. L. (2011). Peer influences on academic motivation: Exploring multiple methods of assessing youths' most "influential" peer relationships. The Journal of Early Adolescence, 31, 13-40. 
Finding The Missing Links, 181

Molm, L. D., Collett, J. L., \& Schaefer, D. R. (2007). Building solidarity through generalized exchange: A theory of reciprocity. American Journal of Sociology, 113, 205-242.

Molm, L. D., Peterson, G., \& Takahashi, N. (1999). Power in negotiated and reciprocal exchange. American Sociological Review, 64, 876-890.

Monroe, S. M., Rohde, P., Seeley, J. R., \& Lewinsohn, P. M. (1999). Life events and depression in adolescence: Relationship loss as a prospective risk factor for first onset of major depressive disorder. Journal of Abnormal Psychology, 108, 606614.

Monroe, W. S. (1898). Social consciousness in children. Psychological Review, 5, 68-70.

Montgomery, J. D. (1992). Job search and network composition: Implications of the strength-of-weak-ties hypothesis. American Sociological Review, 57, 586-596.

Moreno, J. (1934). Who Shall Survive? A New Approach to the Problem of Human Interrelations. New York: Beacon House.

Moreno, J. L. (1951). Sociometry, Experimental Method, and the Science of Society. Ambler, PA: Beacon House.

Mosbach, P., \& Leventhal, H. (1988). Peer group identification and smoking: Implications for intervention. Journal of Abnormal Psychology, 97(2), 238-245.

Muller, C. (1998). Gender differences in parental involvement and adolescents' mathematics achievement. Sociology of Education, 71, 336-356.

Mussen, P. H. (Ed.). (1970). Carmichael's manual of child psychology (3 ${ }^{\text {rd }}$ ed.). New York: Wiley. 
Finding The Missing Links, 182

Neal, J. W. (2008). "Kracking” the missing data problem: Applying Krackhardt's cognitive social structures to school-based social networks. Sociology of Education, 81, 140-162.

Neal, J. W., Cappella, E., Wagner, C., \& Atkins, M. S. (2011). Seeing eye to eye: Predicting teacher-student agreement on classroom social networks. Social Development, 20, 376-393.

Neal, J. W., Neal, Z. P., \& Cappella, E. (2014). I know who are my friends are, but do you? Predictors of self-reported and peer-inferred relationships. Child Development, 85, 1366-1372.

Neal, Z. P., \& Neal, J. W. (2013). Opening the black box of social cognitive mapping. Social Development, 22, 604-608.

Nehnevajsa, J. (1956). Sociometry: Decades of growth. Pp. 48-95 in Sociometry and the Science of Man, edited by Jacob L. Moreno. New York: Beacon in Freeman, M. (2004). The Development of Social Network Analysis. Vancouver, British Columbia: Empirical Press.

Nelson, M. R., \& DeBacker, T. K. (2008). Achievement motivation in adolescents: The role of peer climate and best friends. The Journal of Experimental Education, 76, 170-189.

Newcomb, A. F., \& Bagwell, C. L. (1995). Children's friendship relations: A metaanalytic review. Psychological bulletin, 117, 306. 
Finding The Missing Links, 183

Newcomb, A. F., Bukowski, W. M., \& Pattee, L. (1993). Children's peer relations: A meta-analytic review of popular, rejected, neglected, controversial, and average sociometric status. Psychological Bulletin, 113, 99-128.

Newman, B. M., \& Newman, P. R. (2001). Group identity and alienation: Giving the we its due. Journal of Youth and Adolescence, 30, 515-538.

Newman, M. E. J. (2003). Ego-centered networks and the ripple effect. Social Networks, $25,83-95$.

Newman, M. E. J., Watts, D. J., \& Strogatz, S. H. (2002). Random graph models of social networks. Proc. Natl. Acad. Sci. USA, 99, Suppl. 1:2566-72.

Newman, P. R., \& Newman, B. M. (1976). Early adolescence and its conflict: Group identity vs. alienation. Adolescence, 11, 261-274.

Nichols, J. D., \& White, J. (2014). Friendship cliques: a comparison of the motivational traits of lower/upper track algebra students. Social Psychology of Education, 17, $141-159$.

Noel, H., \& Nyhan, B. (2011). The "unfriending" problem: The consequences of homophily in friendship retention for causal estimates of social influence. Social Networks, 33, 211-218.

Northway, M. (1946). Sociometry and some challenging problems of social relationships. Sociometry, 9, 187-198. 
Finding The Missing Links, 184

Odom, S. L., \& DuBose, R. F. (1981). Peer rating assessments of integrated preschool classes: Stability and concurrent validity of the measures and efficacy of the peer model. In national convention for the Council for Exceptional Children, New York.

Ollendick, T. H., Weist, M. D., Borden, M. C., \& Greene, R. W. (1992). Sociometric status and academic, behavioral, and psychological adjustment: A five-year longitudinal study. Journal of Consulting and Clinical Psychology, 60, 80-87.

Owen, T. (1996). Aristotle: Introductory Readings. Hackett. p. 274.

Pachucki, M. A., Jacques, P. F., \& Christakis, N. A. (2011). Social network concordance in food choice among spouses, friends and siblings. American Journal of Public Health, 101, 2170-7.

Padgett, J. F., \& Ansell, C. K. (1993). Robust action and the rise of the Medici, 14001434. American Journal of Sociology, 98, 1259-1319.

Pajares, F. (1996). Self-efficacy beliefs in academic settings. Review of Educational Research, 66, 543-578.

Pajares, F., \& Schunk, D. H. (2001). Self-beliefs and school success: Self-efficacy, selfconcept, and school achievement. In R. J. Riding, \& S. G. Rayner (Eds.), Self perception (pp. 239-265). Westport, CT: Ablex Publishing in Doll, B., Spies, R. A., LeClair, C. M., Kurien, S. A., \& Foley, B. P. (2010). Student perceptions of classroom learning environments: Development of the ClassMaps Survey. School Psychology Review, 39, 203-218. 
Finding The Missing Links, 185

Pan, L., \& Santos, E.E. (2008). An anytime-anywhere approach for maximal clique enumeration in social network analysis. Systems, Man and Cybernetics, 35293535.

Pappi, F. U., \& Stelck, K. (1987). Ein Databanksystem zur Netzwerkanalyse. 253-265 in Methoden Netzwerkanalyse, edited by Franz Urban Pappi. München: Oldenberg in Freeman, M. (2004). The Development of Social Network Analysis. Vancouver, British Columbia: Empirical Press.

Parker, J. G., \& Asher, S. R. (1993). Friendship and friendship quality in middle childhood: Links with peer group acceptance and feelings of loneliness and social dissatisfaction. Developmental Psychology, 29, 611-621.

Parker, J., \& Asher, S. (1987). Peer relations and later personal adjustment: Are low accepted children “'at risk”? Psychological Bulletin, 102, 357-389.

Parkhurst, J. T., \& Hopmeyer, A. (1998). Sociometric popularity and peer-perceived popularity: Two distinct dimensions of peer status. Journal of Early Adolescence, $18,125-144$.

Pastorelli, C., Caprara, G. V., Barbaranelli, C., Rola, J., Rozsa, S., \& Bandura, A. (2001). The structure of children's perceived self-efficacy: A cross-national study. European Journal of Psychological Assessment, 17, 87-97.

Patacchini, E., \& Zenou, Y. (2008). The strength of weak ties in crime. European Economic Review, 52, 209-236.

Patterson, G. R., DeBaryshe, B. D., \& Ramsey, E. (1989). A developmental perspective on antisocial behavior. American Psychologist, 44, 329-335. 
Finding The Missing Links, 186

Paul, E. L., \& White, K. M. (1990). The development of intimate relationships in late adolescence. Adolescence, 25, 375-400.

Pearl, R., Leung, M. C., Van Acker, R., Farmer, T. W., \& Rodkin, P. C. (2007). Fourthand fifth-grade teachers' awareness of their classrooms' social networks. The Elementary School Journal, 108, 25-39.

Pearson, M. \&, Michell, M. P. L. (2000). Smoke rings: social network analysis of friendship groups, smoking and drug-taking. Drugs: Education, Prevention, and Policy, 7, 21-37.

Pearson, M., \& West, P. (2003). Drifting smoke rings: Social network analysis and Markov processes in a longitudinal study of friendship groups and risk-taking. Connections, 25, 59-76.

Pearson, M., Sieglich, C., \& Snijders, T. (2006). Homophily and assimilation among sport-active adolescent substance users. Connections, 27, 47-63.

Perneger, T. V. (1998). What's wrong with Bonferroni adjustments. BMJ: British Medical Journal, 316, 1236-1241.

Pianta, R. C. (1999). Enhancing relationships between children and teachers. Washington, DC: American Psychological Association.

Pianta, R. C., \& Stuhman, M. W. (2004). Teacher-child relationships and children's success in the first years of school. School Psychology Review, 33, 444-458.

Pijl, S. J., Koster, M., Hannink, A., \& Stratingh, A. (2011). Friends in the classroom: a comparison between two methods for the assessment of students' friendship networks. Social Psychology of Education, 14, 475-488. 
Finding The Missing Links, 187

Plato (1968). Laws. Plato in Twelve Volumes, Vol. 11. Bury translator. Cambridge: Harvard University Press.

Popielarz, P., \& McPherson, J. M. (1995). On the edge or in between: Niche position, niche overlap, and the duration of voluntary memberships. American Journal of Sociology, 101, 698-720.

Poulin, F., \& Dishion, T. J. (2008). Methodological issues in the use of peer sociometric nominations with middle school youth. Social Development, 17, 908-921.

Poulin, F., Dishion, T. J., \& Haas, E. (1999). The peer influence paradox: Friendship quality and deviancy training within male adolescent friendship. Merrill-Palmer Quarterly, 45, 42-61.

Price, D. D. S. (1976). A general theory of bibliometric and other cumulative advantage processes. Journal of the American society for Information science, 27, 292-306.

Prim, R. C. (1957). Shortest connection networks and some generalizations. In: Bell System Technical Journal, 36, 1389-1401.

Proctor, C. H. (1969). Analyzing pair data and point data on social relationships, attitudes and background characteristics of Costa Rican Census Bureau employees. Social Statistics Section, Proceedings of the American Statistical Association, 457-465.

Putallaz, M., \& Wasserman, A. (1989). Children's naturalistic entry behavior and sociometric status: A developmental perspective. Developmental Psychology, 25, 297-305.

R Core Team (2015). R: A language and environment for statistical computing. $R$ Foundation for Statistical Computing. Vienna, Austria. 
Finding The Missing Links, 188

Rapoport, A. (1953a). Spread of information through a population with socio-structural bias: I. Assumption of transitivity. The Bulletin of Mathematical Biophysics. 15, 523-533.

Rapoport, A. (1953b). Spread of information through a population with socio-structural bias: II. Various models with partial transitivity. The Bulletin of Mathematical Biophysics, 15, 535-546.

Reis, O., \& Youniss, J. (2004). Patterns in identity change and development in relationships with mothers and friends. Journal of Adolescent Research, 19, 3144.

Reitzes, D.C., Crimmins, T. J., Yarbrough, J., \& Parker, J. (2011). Social support and social network ties among the homeless in a downtown Atlanta park. Journal of Community Psychology, 39(3), 274-291.

Reynolds, A. J. (1991). The middle schooling process: Influences on science and mathematics achievement from the longitudinal study of American youth. Adolescence, 26, 132-157.

Ricard, R. J., Miller, G. A., \& Heffer, R. W. (1995). Developmental trends in the relation between adjustment and academic achievement for elementary school children in mixed-age classrooms. School Psychology Review, 24, 258-270.

Rice, R. E. (1993). Using network concepts to clarify sources and mechanisms of social influence. Progress in Communication Sciences, 12, 43-62. 
Finding The Missing Links, 189

Richards, M. H., Crowe, P. A., Larson, R., \& Swarr, A. (1998). Developmental patterns and gender differences in the experience of peer companionship during adolescence. Child development, 69, 154-163.

Richards, W. D. (1975). A Manual for Network Analysis (using NEGOPY Network Analysis Program). Stanford: Stanford University.

Richards, W. D. (1995). NEGOPY 4.30 Manual and User's Guide. Burnaby, British Columbia: School of Communication, Simon Fraser University.

Roberts, S. G., \& Dunbar, R. I. M. (2011). Communication in social networks: Effects of kinship, network size, and emotional closeness. Personal Relationships, 18, 43952.

Robins, G., Pattison, P., Kalish, Y., \& Lusher, D. (2007). An introduction to exponential random graph ( $\left.\mathrm{p}^{*}\right)$ models for social networks. Social Networks, 29, 173-191.

Rodkin, P. C., \& Ahn, H. J. (2009). Social networks derived from affiliations and friendships, multi-informant and self-reports: Stability, concordance, placement of aggressive and unpopular children, and centrality. Social Development, 18, 556576.

Rodkin, P. C., Farmer, T. W., Pearl, R., \& Van Acker, R. (2000). Heterogeneity of popular boys: Antisocial and prosocial configurations. Developmental Psychology, 36, 14-24.

Roland, E., \& Galloway, D. (2002). Classroom influences on bullying. Educational research, 44, 299-312. 
Finding The Missing Links, 190

Romney, A. K., Weller, S. C., \& Batchelder, W. H. (1986). Culture as consensus: a theory of culture and informant accuracy. American Anthropologist, 88, 313-342.

Rosenquist, J. N., Murabito, J., Fowler, J. H., \& Christakis, N. A. (2010). The spread of alcohol consumption behavior in a large social network. Annals of Internal Medicine, 152, 426-433.

Rubin, K. H., Bukowski, W. M., \& Parker, J. G. (1998). Peer interactions, relationships, and groups. Handbook of child psychology. In W. Damon (Ed. In Chief), \& N. Eisenberg (Volume Ed.). Handbook of child psychology: Vol. 3 Social, emotional and personality development (5 $5^{\text {th }}$ ed., pp. 619-700). New York: Wiley.

Rubin, K. H., Bukowski, W., \& Parker, J. G. (2006). Peer interactions, relationships, and groups. In W. Damon, \& R. M. Lerner (Series Eds.), \& N. Eisenberg (Vol. Ed.), Handbook of child psychology: Vol. 3. Social, emotional, and personality development (6th ed., pp. 571-645). New York: Wiley.

Rubin, K. H., Bukowski, W. M., Parker, J. G., \& Bowker, J. C. (2008). Peer interactions, relationships, and groups. Handbook of child psychology: Social, emotional, and personality development, 3 .

Rubin, K., LeMare, L., \& Lollis, S. (1990). Social withdrawal in childhood: Developmental pathways to peer rejection. In S. Asher, \& J. Coie (Eds.), Peer rejection in childhood (pp. 217-249). Cambridge, UK: Cambridge Univ. Press.

Runtz, M. G., \& Schallow, J. R. (1997). Social support and coping strategies as mediators of adult adjustment following childhood maltreatment. Child Abuse, \& Neglect, $21,211-226$. 
Finding The Missing Links, 191

Ryan, A. M. (2000). Peer groups as a context for the socialization of adolescents' motivation, engagement, and achievement in school. Educational Psychologist, 35, 101-111.

Ryan, A. M. (2001). The peer group as a context for the development of young adolescent motivation and achievement. Child Development, 72, 1135-1150.

Ryan, B., \& Gross, N. C. (1943). The diffusion of hybrid seed corn in two Iowa communities. Rural Sociology, 8, 15-24.

Ryan, R. M., \& Deci, E. L. (2000). Self-determination theory and the facilitation of intrinsic motivation, social development, and well-being. American Psychologist, 55, 68-78.

Ryan, R. M., Stiller, J. D., \& Lynch, J. H. (1994). Representation of relationships to teachers, parents, and friends as predictors of academic motivation and selfesteem. Journal of Early Adolescence, 14, 226-249.

Sampson, R. J., Morenoff, J. D., \& Gannon-Rowley, T. (2002). Assessing "neighborhood effects": Social processes and new directions in research. Annual Review of Sociology, 28, 443-478.

Sandstrom, M. J., \& Cillessen, A. H. (2006). Likeable versus popular: Distinct implications for adolescent adjustment. International Journal of Behavioral Development, 30, 305-314. 
Finding The Missing Links, 192

Savin-Williams, R. C (1996). Dating and romantic relationships among gay, lesbian, and bisexual youths. In R. C. Savin-Williams, \& K. M. Cohen (Eds.), The lives of lesbians, gays, and bisexuals: Children to adults (pp. 166-180). Fort Worth, TX: Harcourt Brace, \& Co.

Scellato, S., Mascolo, C., Musolesi, M., \& Latora, V. (2010). Distance matters: Geosocial metrics for online social networks. In Proceedings of the 3rd conference on Online social networks (pp. 8-18).

Schaefer, D. R., Simpkins, S. D., Vest, A. E., \& Price, C. D. (2011). The contribution of extracurricular activities to adolescent friendships: New insights through social network analysis. Developmental Psychology, 47, 1141.

Schneider, B. H., Fonzi, A., Tani, F., \& Tomada, G. (1997). A Cross-Cultural Exploration of the Stability of Children's Friendships and the Predictors of their Continuation. Social Development, 6, 322-339.

Schunk, D. H., \& Pajares, F. (2005). Competence perceptions and academic functioning. In A. J. Elliot, \& C. S. Dweck (Eds.), Handbook of competence and motivation (pp. 85-104). New York: Guilford Publications in Doll, B., Spies, R. A., LeClair, C. M., Kurien, S. A., \& Foley, B. P. (2010). Student perceptions of classroom learning environments: Development of the ClassMaps Survey. School Psychology Review, 39, 203-218.

Schuster, B. (2001). Rejection and victimization by peers. In J. Juvonen, \& S. Graham (Eds.), Peer harassment in school: The plight of the vulnerable and the victimized (pp. 290-309). New York: Guilford Press. 
Finding The Missing Links, 193

Scott, J. (2012). Social Network Analysis. Sage Publications.

Scott, W. (1955). Reliability of content analysis: The case of nominal scale coding. Public Opinion Quarterly, 19, 321-325.

Sebanc, A. (2003). The friendship features of preschool children: Links with prosocial behavior and aggression. Social Development, 12, 249-268.

Seginer, R., \& Noyman, M. S. (2005). Future orientation, identity and intimacy: Their relations in emerging adulthood. European Journal of Developmental Psychology, 2, 17-37.

Seidman, S. B., \& Foster, B. L. (1979). SONET-1. Social Networks, 2, 85-90.

Selman, R. L. (1980). The growth of interpersonal understanding: Developmental and clinical analysis. New York: Academic Press.

Serbin, L. A., Moller, L. C., Gulko, J., Powlishta, K. K., \& Colburne, K. A. (1994). The emergence of gender segregation in toddler playgroups. New Directions for Child and Adolescent Development, 1994, 7-17.

Sessa, F. M. (2007). Peer crowds in a commuter college sample: The relation between self-reported alcohol use and perceived peer crowd norms. The Journal of Psychology, 141, 293-305.

Seyfarth, R. M., \& Cheney, D. L. (2012). The evolutionary origins of friendship. Annual Review of Psychology, 63, 153-177.

Shalizi, C. R., \& Thomas, A. C. (2011). Homophily and contagion are generically confounded in observational social network studies. Sociological Methods and Research, 40, 211-239. 
Finding The Missing Links, 194

Shin, H. (2014). Examining Peer Selection and Influence Processes on Early

Adolescents' Academic Adjustment with Longitudinal Social Network Analysis

(Doctoral dissertation, Pennsylvania State University).

Shin, H., \& Ryan, A. M. (2014). Early adolescent friendships and academic adjustment:

Examining selection and influence processes with longitudinal social network analysis. Developmental Psychology, 50, 2462-2472.

Shrum, W., \& Cheek, N. H. (1987). Social structure during the school years: Onset of the degrouping process. American Sociological Review, 52, 218-223.

Shrum, W., Cheek Jr, N. H., \& MacD, S. (1988). Friendship in school: Gender and racial homophily. Sociology of Education, 61, 227-239.

Shutts, K., Roben, C. K. P., \& Spelke, E. S. (2013). Children's use of social categories in thinking about people and social relationships. Journal of Cognition and Development, 14, 35-62.

Sieving, R. E., Eisenberg, M. E., Pettingell, S., \& Skay, C. (2006). Friends' influence on adolescents' first sexual intercourse. Perspectives on Sexual and Reproductive Health, 38, 13-19.

Simmel, G. (1908/1971). On Individuality and Social Forms. Chicago: University of Chicago in Freeman, M. (2004). The Development of Social Network Analysis. Vancouver, British Columbia: Empirical Press.

Simons-Morton, B. G., \& Crump, A. D. (2003). The association of parental involvement and social competence with school adjustment and engagement among sixth graders. Journal o School Health, 73, 121-126. 
Finding The Missing Links, 195

Simons-Morton, B., \& Chen, R (2009). Peer and parent influences on school engagement among early adolescents. Youth, \& Society, 41, 3-25.

Simpson, B., \& Borch, C. (2005). Does power affect perception in social networks? Social Psychology Quarterly, 68, 278-287.

Simpson, B., Markovsky, B., \& Steketee, M. (2011). Power and the perception of social networks. Social Networks, 33, 166-171.

Sinclair, M. F., Christenson, S. L., Lehr, C. A., \& Reschly-Anderson, A. (2003). Facilitating school engagement: Lessons learned from Check, \& Connect longitudinal studies. The California School Psychologist, 8, 29-41.

Singh, J. (2005). Collaborative networks as determinants of knowledge diffusion patterns. Management Science, 51, 756-770.

Singh, K., Granville, M., Dika, S. (2002). Mathematics and Science Achievement: Effects of Motivation, Interest, and Academic Engagement. Journal of Educational Research, 95, 323-332.

Siu, S.-F. (1994). Taking no chances: A profile of a Chinese-American family's support for school success. Equity and Choice, 10, 23-32.

Skilton, P. F. (2008). Similarity, familiarity and access to elite work in Hollywood: Employer and employee characteristics in breakthrough employment. Human Relations, 61, 1743-1773.

Skinner, E. A., \& Zimmer-Gembeck, M. J. (2007). The development of coping. Annual Review of Psychology, 58, 119-144. 
Finding The Missing Links, 196

Smith, A. B., \& Inder, P. M. (1993). Social interaction in same and cross gender preschool peer groups: A participant observation study. Educational Psychology, $13,29-42$.

Smith, G. C., \& Pell, J. P. (2003). Parachute use to prevent death and major trauma related to gravitational challenge: Systematic review of randomised controlled trials. BMJ: British Medical Journal, 327, 1459.

Snijders, T. (2011). Statistical models for social networks. Annual Review of Sociology, 37, 131-153.

Snijders, T. A. B., Steglich, C. E. G., Schweinberger, M., \& Huisman, M. (2005). Manual for SIENA version 2.1. Groningen: ICS, Department of Sociology.

Snijders, T. A., \& Borgatti, S. P. (1999). Non-parametric standard errors and tests for network statistics. Connections, 22, 161-170.

Snijders, T., Steglich, C., \& Schweinberger, M. (2007). Modeling the coevolution of networks and behavior (pp. 41-71) in Longitudinal Models in the Behavioral and Related Sciences, edited by van Montfort, K., Oud, H., \& Satorra, A. Mahwah, NJ: Lawrence Erlbaum.

Steglich, C., Snijders, T. A., \& Pearson, M. (2010). Dynamic networks and behavior: Separating selection from influence. Sociological Methodology, 40, 329-393.

Steinberg, L., \& Silverberg, S. B. (1986). The vicissitudes of autonomy in early adolescence. Child Development, 57, 841-851. 
Finding The Missing Links, 197

Steinberg, L., Lamborn, S. D., Dornbusch, S. M., \& Darling, N. (1992). Impact of parenting practices on adolescent achievement: Authoritative parenting, school involvement, and encouragement to succeed. Child Development, 63, 1266-1281.

Steinfield, C., Ellison, N. B., \& Lampe, C. (2008). Social capital, self-esteem, and use of online social network sites: A longitudinal analysis. Journal of Applied Developmental Psychology, 29, 434-445.

Stipek, D. J., \& Hoffman, J. M. (1980). Children's achievement-related expectancies as a function of academic performance histories and sex. Journal of Educational Psychology, 72, 861-865.

Strayer, F. F., \& Strayer, J. (1976). An ethological analysis of social agonism and dominance relations among preschool children. Child Development, 47, 980-989.

Sudman, S. (1988). Experiments in measuring neighbor and relative social networks. Social Networks, 10, 93-108.

Sudman, S. (1988). Experiments in measuring neighbor and relative social networks. Social Networks, 10, 93-108.

Sugimura, K., \& Shimizu, N. (2010). The role of peers as agents of identity formation in Japanese first-year university students. Identity: An International Journal of Theory and Research, 10, 106-121.

Sullivan, H. S. (1953). The interpersonal theory of psychiatry. New York: Norton.

Sussman, S., Dent, C. W., Stacy, A. W., Burciaga, C., Raynor, A., Turner, G. E., et al. (1990). Peer group association and adolescent tobacco use. Journal of Abnormal Psychology, 99, 349-352. 
Finding The Missing Links, 198

Sussman, S., Pokhrel, P., Ashmore, R. D., Brown, B. B. (2007). Adolescent peer group identification and characteristics: A review of the literature. Addictive Behaviors, 32, 1602-1627.

Taylor, M. (1999). Imaginary companions and the children who create them. Oxford University Press.

Teachman J. D., Paasch K. M., Day R. D., \& Carver K. P. (1996). Poverty during adolescence and subsequent educational attainment. In Duncan, Brooks-Gunn (Eds.), Consequences of Growing Up Poor. New York: Russell Sage Foundation, pp 340-381.

Terry, R., \& Coie, J. D. (1991). A comparison of methods for defining sociometric status among children. Developmental Psychology, 27, 867-881.

The Organization for Economic Cooperation and Development (OECD; 2010). "PISA

2009 Results: What Students Know and Can Do: Student Performance in Reading, Mathematics and Science (Volume I)". Paris, France.

Thompson, G., \& Powell, M. (1951). An investigation of the rating scale approach to the measurement of social status. Educational and Psychological Measurement, 11, 440-455.

Thorne, B. (1992). Girls and boys together... but mostly apart: Gender arrangements in elementary schools. In J. Wrigley (Ed.), Education and gender equality. London, UK: Falmer Press. Pp. 115-130.

Tokar, A. (2009). Metaphors of the Web 2.0: With special emphasis on social networks and folksonomies. Frankfurt: Peter Lang. p. 57. 
Finding The Missing Links, 199

Tönnies, F. (1855/1936). Gemeinschaft und Gesellschaft. East Lansing, MI: Michigan State University in Freeman, M. (2004). The Development of Social Network Analysis. Vancouver, British Columbia: Empirical Press.

Tooker, E. (1983). The structure of the Iroquois League: Lewis H. Morgan's research and observations. Ethnohistory, 30, 141-154.

Travers, J., \& Milgram, S. (1969). An experimental study of the small world problem. Sociometry, 32, 425-443.

Tremblay, C., Hébert, M., \& Piché, C. (1999). Coping strategies and social support as mediators of consequences in child sexual abuse victims. Child Abuse \& Neglect, 23, 929-945.

Tsai, W., \& Ghoshal, S. (1998). Social capital and value creation: The role of intrafirm networks. Academy of Management Journal, 41, 464-476.

Tuma, N. B., \& Hallinan, M. Z. (1979). The effects of sex, race and achievement on schoolchildren's friendships. Social Forces, 57, 1265-1285.

Turner, J. C., Midgley, C., Meyer, D. K., Gheen, M., Anderman, E. M., Kang, Y., et al. (2002). The classroom environment and students' reports of avoidance strategies in mathematics: A multimethod study. Journal of Educational Psychology, 94, 88-106.

U. S. Department of Education, National Center for Education Statistics, Common Core of Data (CCD), "Public Elementary/Secondary School Universe Survey," 2009_ 10, Version Provisional 2a. 
Finding The Missing Links, 200

Urberg, K. A. (1992). Locus of peer influence: Social crowd and best friend. Journal of Youth and Adolescence, 21, 439-450.

Urberg, K. A., Değirmencioğlu, S. M., Tolson, J. M., \& Halliday-Scher, K. (1995). The structure of adolescent peer networks. Developmental Psychology, 31, 540-547.

Urberg, K. A., Değirmencioğlu, S. M., Tolson, J. M., \& Halliday-Scher, K. (2000). Adolescent social crowds: Measurement and relationship to friendships. Journal of Adolescent Research, 15, 427-445.

Valente, T. W. (1995). Network Models of the Diffusion of Innovations. Cresskill, NJ: Hampton Press.

Valente, T. W. (1996). Social network thresholds in the diffusion of innovations. Social Networks, 18, 69-89.

Valente, T. W., Hoffman, B. R., Ritt-Olson, A., Lichtman, K., \& Johnson, C. A. (2003). Effects of a social-network method for group assignment strategies on peer-led tobacco prevention programs in schools. American Journal of Public Health, 93, $1837-1843$.

Valente, T. W., Palinkas, L. A., Czaja, S., Chu, K. H., \& Brown, C. H. (2015). Social network analysis for program implementation. PloS one, 10, e0131712.

Vaughn, B. E., \& Waters, E. (1981). Attention structure, sociometric status, and dominance: Interrelations, behavioral correlates, and relationships to social competence. Developmental Psychology, 17, 275-288. 
Finding The Missing Links, 201

Vaughn, B. E., Azria, M. R., Krzysik, L., Caya, L. R., Bost, K. K., Newell, W., \& Kazura, K. (2000). Friendship and social competence in a sample of preschool children attending head start. Developmental Psychology, 36, 326-338.

Verbrugge, L. M. (1977). The structure of adult friendship choices. Social Forces, 56, $576-597$.

Verbrugge, L. M. (1983). A research note on adult friendship contact: A dyadic perspective. Social Forces, 62, 78-83.

Verhoeven, K. J., Simonsen, K. L., \& McIntyre, L. M. (2005). Implementing false discovery rate control: Increasing your power. Oikos, 108, 643-647.

Visser, P. S., \& Mirabile, R. R. (2004). Attitudes in the social context: The impact of social network composition on individual-level attitude strength. Journal of Personality and Social Psychology, 87, 779-795.

Vitaro, F., Brendgen, M., \& Tremblay, R. E. (2000). Influence of deviant friends on delinquency: Searching for moderator variables. Journal of Abnormal Child Psychology, 28, 313-325.

Voelkl, K. E. (1997). Identification with school. American Journal of Education, 105, 204-319.

von Eye, A. (1990). Introduction to configural frequency analysis: The search for types and antitypes in cross-classifications. New York: Cambridge University Press.

Watts, D. J. (1999). Networks, dynamics, and the small-world phenomenon. American Journal of Sociology, 105, 493-527. 
Finding The Missing Links, 202

Watts, D. J. (2003). Six degrees: The science of a connected age. New York: W. W. Norton.

Watts, D.J., \& Strogatz, S.H. (1998). Collective dynamics of 'small-world' networks. Nature, 393, 440-442.

Webb, H. J., \& Zimmer-Gembeck, M. J. (2014). Body image and body change strategies within friendship dyads and groups: Implications for adolescent appearance-based rejection sensitivity. Social Development, 24, 1-19.

Welker, W. (1977). Humor in education: A foundation for wholesome living. College Student Journal, 11, 252-254.

Wellman, B. (1926). The school child's choice of companions. The Journal of Educational Research, 14, 126-132.

Wentzel, K. R. (1991). Relations between social competence and academic achievement in early adolescence. Child Development, 62, 1066-1078.

Wentzel, K. R. (2002). Are effective teachers like good parents? Teaching styles and student adjustment in early adolescence. Child Development, 73, 287-301.

Wentzel, K. R., \& Asher, S. R. (1995). The academic lives of neglected, rejected, popular, and controversial children. Child Development, 66, 754-763.

Wentzel, K. R., \& Caldwell, K. (1997). Friendships, peer acceptance, and group membership: Relations to academic achievement in middle school. Child Development, 68, 1198-1209.

Wentzel, K. R., \& Watkins, D. (2002). Peer relationships and collaborative learning as contexts for academic enablers. School Psychology Review, 31, 366-377. 
Finding The Missing Links, 203

Wentzel, K. R., McNamara-Barry, C., \& Caldwell, K. A. (2004). Friendships in middle school: Influences on motivation and school adjustment. Journal of Educational Psychology, 96, 195-203.

Werner, N. E., \& Crick, N. R. (2004). Maladaptive peer relationships and the development of relational and physical aggression during middle childhood. Social Development, 13, 495-514.

Wu, J. C., Chen, C. L., Hou, M. C., Chen, T. Z., Lee, S. D., \& Lo, K. J. (1994). Multiple viral infection as the most common cause of fulminant and subfulminant viral hepatitis in an area endemic for hepatitis B: Application and limitations of the polymerase chain reaction. Hepatology, 19, 836-840.

Xie, H., Cairns, R. B., \& Cairns, B. D. (1999). Social networks and configurations in inner-city schools: Aggression, popularity, and implications for students with EBD. Journal of Emotional and Behavioral Disorders, 7, 147-156.

Xie, H., Swift, D. J., Cairns, B. D., \& Cairns, R. B. (2002). Aggressive behaviors in social interaction and developmental adaptation: A narrative analysis of interpersonal conflicts during early adolescence. Social Development, 11, 205224.

$\mathrm{Xu}, \mathrm{R}$. (2003). Measuring explained variation in linear mixed effects models. Statistics in Medicine, 22, 3527-3541.

Yao, E. L. (1985). A comparison of family characteristics of Asian American and AngloAmerican high achievers. International Journal of Comparative Sociology, 26, $198-207$. 
Finding The Missing Links, 204

Yazzie-Mintz, E. (2010). Charting the path from engagement to achievement: A report on the 2009 High School Survey of Student Engagement. Bloomington, IN: Center for Evaluation, \& Education Policy.

Yugar, J. M., \& Shapiro, E. S. (2001). Elementary children's school friendship: A comparison of peer assessment methodologies. School Psychology Review, 30, 568-585.

Zimmer-Gembeck, M. J. (1999). Stability, change and individual differences in involvement with friends and romantic partners among adolescent females. Journal of Youth and Adolescence, 28, 419-438.

Zipf, G. K. (1949). Human Behavior and the Principle of Least Effort. Menlo Park, CA: Addison-Wesley. 
Finding The Missing Links, 205

\section{APPENDIX A: A History of Social Network Analysis}

Although not necessary to appreciate this study, the history of social network analysis is a fascinating and interconnected endeavor, and the study of it can provide some helpful background on the current methods being examined. Freeman has written an excellent book on the topic (2004). This section represents a distillation of his work, along with some additional thoughts on the development of the mathematical side of the method.

\section{Development of the Social Network Perspective}

In Freeman's search of early social networks researchers (2004), he found that Auguste Comte was one of the first researchers to explicitly describe a network perspective on human relationships. Comte was born in Montpelier, France in 1798 and, by 1830 , began publishing a series of papers on sociology (a word coined by him). He felt that the purpose of sociology was to uncover the laws of society, and identified the two main aspects of the field as statics and dynamics. He wrote that statics were focused on the laws of social interconnection or the laws of action and reaction of the different parts of the social system (Martineau, 1835/2000 as cited in Freeman, 2004). Comte felt that the family, not the individual, was the most fundamental level, arguing that families grew into tribes, and tribes eventually grew into nations. Thus, to Comte, society was made up of several layers of networks.

This intuition is not unique to Comte. Freeman reproduced a descent list from the Book of Genesis (Freeman, 1989, p. 18), suggesting "that human beings have, since the 
Finding The Missing Links, 206

earliest days, [implicitly] recognized the importance of the ties that link social actors." (Freeman, 2004, p. 10). Similarly, Hawaiian royalty contained specialists whose role was to memorize the genealogies of current and previous monarchs, preserving the knowledge of ancestral origins (Kirch, 2010). Nonetheless, Comte was among the first to explicitly argue for the importance of social networks in the lives of people. His argument quickly caught wind.

Several sociologists viewed connections as a way to distinguish traditional versus modern societies. Ferdinand Tönnies (1855/1936, in Freeman, 2004) used the word gemeinschaft to describe traditional societies, in which social ties were based on shared values and beliefs, and the word gesellschaft to describe modern societies, where social ties were often formal, instrumental, and impersonal. Emile Durkheim (1893/1964, in Freeman, 2004) viewed traditional societies as containing rigid arrangements that linked individuals into repressive networks, while he viewed modern societies as made up of a division of labor that led individuals to form cooperative links.

Gustave LeBon espoused an entirely different structural perspective (1897/1995), arguing that when individuals amass in crowds, they lose their individual identities and imitate others around them. Thus, behaviors and ideas spread from person to person by a process he called "contagion." LeBon's idea was quite innovative, as contagion remains an active area of research, being invoked to explain the spread of new technology by leaders (Iyengar, Van den Bulte \& Valente, 2010), financial confidence (Corsetti, Pericoli, \& Sbracia, 2010), and emotions under strong test conditions (Lishner, Cooter, \& Zald, 2008). See Appendix G for a brief discussion of recent work in this area. 
Finding The Missing Links, 207

Perhaps the most direct innovator of SNA after Comte was Georg Simmel, who wrote that "society exists where a number of individuals enter into interaction" (1908/1971, p. 23 in Freeman, 2004). He argued that a collection of human beings did not simply become a society because each individual "has an objectively determined or subjectively impelling life-content", that individuals are essentially moving in their own direction. A society can only exist when there are connections between individuals, allowing for some sort of reciprocal influence, leading to a sort of collective motivation, which becomes the direction of society. Thus, to Simmel, to study society, researchers had to study the connections between the individuals that societies are made up of.

\section{Development of the Social Network Method}

Without a procedure, SNA was merely a nice idea. Pierre Huber expanded the SNA approach by introducing the study of systematic empirical data. Pierre Huber assisted his father, François Huber, with studying the social structure of honeybees by systematically examining patterns of social dominance (Huber \& Bonnet, 1792 in Freeman, 2004). More than fifty years later, the first systematic data collection on the social structure of humans would be completed on the Iroquois in New York by Henry Morgan, who desired to understand more about their society in order to model his secret society off of them. He later became so engrossed in them he published an ethnography (Tooker, 1983). In his research, he discovered that the Iroquois descent system was curiously far different from the European system, sparking a career in the study of Native American descent systems. 
Finding The Missing Links, 208

John Atkinson Hobson extended this study of social patterns to a very specific portion of contemporary society: the links between businesses (1884/1954). Hobson uncovered several businessmen who sat on the boards of multiple companies, giving them advantages by coordinating both companies. In his presentation of his findings, Hobson produced two innovations. First, he presented the links by using a table in which businessmen were listed along the side and businesses were listed along the top. He then indicated businessmen who sat on the boards of multiple companies by noting a "1" under each company in the row with the individual. Second, he produced a graphic that would become a forerunner of the modern network graph: circles representing each company that overlapped when they shared board members. Through his data and this graph, Hobson easily demonstrated that a plethora of companies were controlled by, or under the influence of, just a handful of businesses.

Educational psychologists then took up the social networks mantle and produced more innovations. John C. Almack measured the social networks of children by asking children which others they would invite to a hypothetical party, and measured the similarities between the nominated students and the ones who nominated them (1922 in Freeman, 2004). He found a remarkable degree of overlap and helped develop the notion that social choices are made, in part, due to similar characteristics, or that individuals tend to seek homophily when making their social networks.

Beth Wellman took a different approach, and systematically observed children who played together during free play (1926), directly extending Huber's approach to humans. Helen Bott then built on Wellman's method in three key ways (1928): first, she 
Finding The Missing Links, 209

chose a focal child to observe. Second, she made note of only key behaviors which had been decided ahead of time, instead of noting all behaviors. Third, she recorded her data in matrix form.

Thus, educational psychologists introduced two major innovations to the method of social networks analysis in a short span of time. Almack introduced the use of questionnaires to map out social networks, while Wellman and Bott helped develop the use of systematic observations. Both methods are important and fraught with their own advantages and disadvantages, however the field has yet to come to a consensus on which basic method should be preferred (Marsden, 1990).

\section{Graph Theory and Social Network Analysis}

Up to this point, mathematics was not particularly utilized by social networks scientists. Decisions about what constituted a social group or a tie were made by the researcher's intuition or by pre-planned criteria, not by any mathematical model. Furthermore, once a network graph was constructed, they were not generally exposed to any rigorous analyses. However, this was not due to a lack of such analyses, as more than 150 years of work had already been done in the field of graph theory, which considered the relationships between the very elements of a network graph: the node and the edge.

The origins of graph theory are often traced to a paper published by Leonhard Euler in 1736, who set out to resolve a simple problem: the city of Königsberg (now Kaliningrad, Prussia) is divided in half by the Pregel River, and in this river are two islands. Seven bridges link the two islands with both sides of the river and each other. 
Finding The Missing Links, 210

The question was simple: could a person traverse each bridge without taking the same bridge twice? Euler's ingenious approach was to reduce the topography of the city to a network: he reduced the four land masses to nodes, and he reduced the bridges to lines, or “edges". Through this graph and the subsequent calculations, he proved that there was no path which satisfied the question (Alexanderson, 2006).

More than one century later, Arthur Cayley expanded on the field of graph theory by studying the properties of many types of graphs (Harary \& Palmer, 1973) and how to enumerate them (given a certain graphical structure, how many subgraphs which meet certain conditions are possible?). This was an important question, as certain kinds of graphs have specific properties, and due to the principle of hierarchy, if a graph can be proven to be made up of smaller graphs which satisfy certain conditions, the overall graph will be known to have the same property as the smaller graphs. Such work led to algorithms used in modern research (for example, Pan and Santos' [2008] approach to the maximal-clique enumeration problem: in a network, how do we determine the largest subgroup in which all members are connected to one another?).

\section{"Who Shall Survive?" and the New Approach}

Social networks analysis and graph theory would come to a head in the research of Jacob Moreno. A Romanian immigrant and psychiatrist who spent his career in the field of SNA, Moreno was an avid writer, and published several books detailing his thoughts about social networks analysis and the composition of society. His most influential was Who Shall Survive? (1934), a book in which Moreno discussed his 
Finding The Missing Links, 211

research on an epidemic of runaways at a boarding school for girls. He shared his method of using questionnaires to measure the social networks of the girls, the characteristics of the participants he measured (such as positive and negative emotional choices, interaction patterns, and kinship ties), and he presented network graphs of the student population. All of these techniques are still used by network analysts today, virtually unchanged.

In the first edition, social networks were computed intuitively, without the aid of a mathematical model. In subsequent editions, he was assisted by mathematical sociologist Paul Lazarsfeld, who used a probabilistic approach to determine which links were reliable and which were not, starting with a baseline model in which all links were chosen at random (Freeman, 2004). This mathematical work would have two big impacts on the field.

First, the increased popularity of SNA spurred by Moreno's work led to further development in graph theory approaches to calculating the properties of a network. The problem of graphical enumeration (the origins of which were previously discussed) was tackled, with major contributions by Harary and Palmer (1973). Several researchers focused on various types of paths one could take through a network, such as the shortest path (Bellman, 1958; Dijkstra, 1959; Hart, Nilsson, \& Raphael, 1968), the shortest path if nodes are separated by physical distance (Held \& Karp, 1962), and the paths that connect all nodes (Kruskal, 1958; Prim, 1957). Other researchers focused on forbidden graph characterization problems, which were related to determining the structure of a network (Beineke, 1968; Ghouila-Houri, 1962). 
Finding The Missing Links, 212

Second, Lazarsfeld's work contributed to the development of probabilistic approaches to graph theory. In 1959, two papers were published independently of each other, proposing a method to tackle a problem in networks analysis: how can researchers account for random and probabilistic processes in networks? In both papers, the authors proposed networks with randomly connected nodes and set out to determine their properties, such as how to compute the probability of an edge between any given pair of nodes (Erdős \& Rényi, 1959; Gilbert, 1959). Such graphs are often used as a sort of "null graph" to compare an empirical graph with. If the graphs agree, the empirical graph is probably generated through mostly random processes. If they disagree, the empirical graph may have some sort of structure worth determining (Newman, Watts, \& Strogatz, 2002).

Another application of random graphs is in the analysis of extremely large graphs, which often require an unfeasibly large amount of computing power to analyze (Aiello, Chung, \& Lu, 2000). According to Jiri Nehnevajsa (1956), Lazarsfeld's work with Moreno and the resulting model "...stands at the roots of probabilistic analysis in the field [of social network analysis].” (p. 61). The relationship between mathematics and SNA was solidified by then, as Freeman notes: "from the start, contributions to social network analysis were often couched in mathematical terms" (2004, p. 147). Likely more so than most other fields of social research, mathematics continues to have a strong influence in social network analysis. 
Finding The Missing Links, 213

\section{Modern Social Network Analysis}

Today, the SNA approach is thriving. There are several network-centric computer programs, such as SONET (Seidman \& Foster, 1979), NEGOPY (Richards, 1975), SONIS (Pappi \& Stelck, 1987), and UCINET (Borgatti, Everett, \& Freeman, 2002). An organization for social networks researchers called the International Network for Social Network Analysis has been operating since 1977, with its own newsletter called Connections (Freeman, 2004). The SNA crowd has several journals solely dedicated to network analysis, such as Social Networks, the Journal of Social Structure, Social Network Analysis and Mining, the International Journal of Social Network Mining, and Social Network Analysis.

The considerations of today do not involve the basic structure of the field, but instead in refining the method in ways to fill certain niches (for example, in a wonderful case of returning to its roots, using current social network research methodology in studying the networks of non-human animals [Sih, Hanser, \& McHugh, 2009]). Several data collection and network analytic methods have been designed for precisely that reason: to capitalize on the types of data available and to create the types of networks most relevant to researchers' questions. 
Finding The Missing Links, 214

APPENDIX B: Network Data Collection Methods

In gathering data for social networks in schools and elsewhere, three

considerations must be made: how network members will be found, how network information will be obtained, and which types of ties will be measured (Hanneman \& Riddle, 2005). There are no set standards for any of these; all are dependent on the population of interest and the resources at the researcher's disposal.

\section{Sampling Strategies}

To identify participants, four methods are commonly used: the saturated network, snowballing, egocentric networks with alter connections, and egocentric networks without alter connections (Hanneman \& Riddle, 2005).

Saturated networks. To obtain a saturated network, the researcher must contact as many members of the population of interest as possible and ask who they associate with. This is obviously most desirable, as it gives the clearest picture. In addition, having the entire network is necessary for any statistics dealing with the network as a whole (such as distance, centrality, and positional equivalence) as well as others dealing with individuals (such as betweenness). Obtaining the saturated network is simplest when the population is known and well-defined (such as all students in a particular school, all registered members of a club, or all employees of a company).

However, if the population is large, obtaining information on all individuals can be expensive and time-consuming. Furthermore, if the population is hidden (such as gangs), identifying who to contact may be impossible. Due to these difficulties, 
Finding The Missing Links, 215

researchers in schools rarely gather data from all members of the population (though not always. For example, researchers in Sweden gathered social network information from $100 \%$ of a population of 14-15 year old students [Määttä, Stattin, \& Nurmi, 2006]), although they can achieve fairly high sampling rates from middle- and high-school students such as gathering social networks data from $88.8 \%$ of the student population (Espelage, Holt, \& Henkel, 2003), 90.1\% (Gest, Domitrovich, \& Welsh, 2005), and 93.5\% (Ellis \& Zarbatany, 2007). However, lower sampling rates are more common, such as 68.1\% (Xie, Swift, Cairns, \& Cairns, 2002), 68.0\% (Farmer et al., 2008), 64.5\% (Estell, Farmer, Cairns, \& Clemmer, 2003), 61.3\% (Duffy \& Nesdale, 2009) and even $48.0 \%$ (Xie et al., 1999). Due to the co-dependent nature of social networks a near census is required to accurately present the structure of the network. Thus, the accuracy of a saturated network can suffer at what would conventionally be considered modest sampling rates.

Furthermore, the resulting saturated network should not be regarded as a network of the population, but rather as a network of the respondents (unless, of course, the entire population was sampled). This is notable when there are differences between the respondents and non-respondents, as it can result in a biased network. For example, in Gest and colleagues' study (2005), the researchers gathered social network data from elementary school students over a period of years. However, they found that nonrespondents and students who dropped out of the study tended to be rated by their teachers as having "weaker academic skills and more aggressive and disruptive behavior 
Finding The Missing Links, 216

problems" than students who participated and stayed with the study. The researchers noted that the effect size of the differences ranged from 0.25 to 0.50 .

Snowballing. The snowballing method is a respondent-driven method that was initially developed to measure social network relationships (Coleman, 1958; Goodman, 1961), but has since grown into favor with researchers of difficult-to-reach populations (such as criminals or the homeless). The method begins with the researcher identify a few "seed" participants who they can directly recruit. Each participant is then given uniquely numbered coupons used to recruit others. New recruits are then given new coupons with which to recruit still more, and so on. All recruits are compensated for participating in the study and for recruiting new participants. The coupon method limits the amount of people an individual can recruit, which accomplishes two things: it limits the bias a person with a large or unique subgroup can induce on the sample, and it encourages a long chain of referrals.

A major assumption of this method is that "if referral chains are sufficiently long, the composition of the final sample with respect to key characteristics and behaviors will become independent of the seeds from whom it began" (Kogan, Wejnert, Chen, \& Brody 2011, p. 34), that the sample characteristics and network structure will resemble the population after enough waves. As an example, imagine a population in which male members will refer other males $70 \%$ of the time and other females $30 \%$ of the time and that the researchers choose only males for the seeds. In the first recruitment waves, the sample will probably be heavily biased towards males, and will probably be more than 
Finding The Missing Links, 217

$70 \%$ male. However, according to the law of large numbers, over enough waves, the proportion of males and females should settle around their expected values, $70 \%$ and $30 \%$, respectively. Ideally, the seeds will be as heterogeneous as possible within the population, to capture the widest variety of participants. However, a "ripple effect" provides a challenge to this assumption (see Newman, 2003).

Using the snowballing method, there are two potential sources of bias (Kogan et al., 2011). The first is a differential network size bias, in which large subgroups can bias the sample. The second is a differential recruitment effectiveness bias, which is another bias caused by recruitment effectiveness varying by group. For example, if a researcher was mapping a social network of individuals with anxiety disorders, those with social phobia might be relatively ineffective at recruiting others with social phobia, biasing the sample network against individuals with social phobia.

Although the snowballing method is a helpful strategy for measuring hidden populations, it does contain some drawbacks (Hanneman \& Riddle, 2005). The first is that this method will not be able to identify isolates. A second drawback is that it may overstate the connectedness and solidarity of populations, if recruiters only recruit people they know and are similar to (biasing the network toward homophily).

Ego-centric networks. Ego-centric networks are networks which revolve around some focal actor (called "ego"). Their construction is simple enough; researchers ask each participant who is in their social network. The researchers can go one step further and ask the participant to note who in their network is connected to one another 
(Hanneman \& Riddle, 2005). An assumption with this method is that all ego-centric networks are independent of one another.

Unless the population under study is densely connected, ego-centric measurements are not likely to yield a picture of the overall network. As such, networkwide measures, such as network density and modularity, will not be available. However, as each ego-centric network is a sample of the overall population network, some of these network-wide measures may be estimated (depending on the quantity of information collected by the researchers. Obviously, more analyses may be performed when participants give the connections between their network members). In addition, such networks still give a sense of the kind of networks people are embedded in, such as how many people individuals are connected to and how close-knit the networks are.

Ego-centric networks can be helpful when the unit of analysis is not the connection between individuals, but the individual herself. For example, Jansson and Spreen (1998) studied the ego-centric networks of heroin users who attended and did not attend regular drug assistance sessions to determine differences in social contact. Meanwhile, Christakis and Fowler (2007) used ego-centric network information to determine an individual's risk of becoming obese by how many people in their personal network were obese and what kind of relationship they had with ego.

\section{Collection Methods}

With the participants selected, there are three common methods for determining participants' network data: administering surveys, conducting observations, and using 
Finding The Missing Links, 219

archived information. Each carries their own pitfalls and has appropriate circumstances for use.

Surveys. By far the most common method, surveys of participants' social networks are relatively inexpensive and simple to analyze. However, as surveys rely on participants to self-disclose their personal networks, surveys must be designed to elicit this as well as possible, and as accurately and reliably as possible. To this end, there is an important consideration in the use of surveys: whether networks should be based on free recall or on name recognition from a premade list.

In social network studies, researchers most often request participants to freely recall network members, such as friends or acquaintances (Masden, 1990). However, this method carries a risk that people may forget network members, resulting in an incomplete network. The amount of network members who are forgotten and the effects this has on the resultant personal network has been studied.

Several researchers have examined the accuracy of the free recall of network members in college students, first by asking participants to freely recall friends and acquaintances, then by showing participants a list of students enrolled in the college and asking the participants who, if anyone, they forgot about. Using this method, Hammer found that participants freely recalled only $79 \%$ of persons they knew very well, saw more than once per week, and saw in the last week (1984). Sudman found somewhat better recall rates, with participants accurately remembering $61 \%$ of their acquaintances, $91 \%$ of their friends, and $92 \%$ of their close friends (1988). A study of students living in a university dormitory examined students' ability to recall friends who also lived in the 
Finding The Missing Links, 220

dormitory. The recall rate was similar to the other studies, with students freely remembering $80 \%$ of their friends who lived in the dormitory (Brewer \& Webster, 1999).

Other research has focused on more formal relationships and found similar results. Members of a student government at the University of Ljubljana (in Slovenia) were asked to freely recall who else on the student government they informally discussed government matters, before being presented with a list of all members of the student government. On average, student government members freely recalled $81 \%$ of fellow government members that they informally discussed government matters with (Hlebec, 1993). In the University of California, Irvine a researcher asked graduate students to name other students in their program. The average student named about $81 \%$ of the fellow students, with more chronologically distant cohorts being forgotten more often (Brewer, 1993). In the public relations department of a southwestern U.S. university, a researcher asked employees to name other employees, and the average employee was able to with a $75 \%$ accuracy rate. No study found any significant demographic differences in recall, nor any consistent demographical characteristics of those who were forgotten.

Others who were remembered by participants tended to be more central in personal networks (Brewer, 2000). Hammer found that the most important characteristics to being remembered was that others be close to the participant, seen often, has a reciprocal relationship (i.e. the participant and the other both regard each other as friends), and has long-lasting interactions (1980; 1984). 
Finding The Missing Links, 221

Thus, on average, friends were freely recalled with an accuracy rate of $80-90 \%$, acquaintances $60 \%$, and co-workers and fellow students $75-80 \%$. As such, it may be more prudent to regard freely recalled friends and co-workers not as a complete list of all people known by the participant, but a sample (Hammer, 1984).

Due to the inaccuracies in recall, and partially due to the differences between those recalled and those forgotten, there are differences in the personal networks of participants when they are based on free recall or on recognition from a list. Brewer and Webster found differences in the networks' number of cliques, betweenness/closeness/ degree/information centralization, and closeness centralities for individuals (1999). However, network size and density tends to hold up well whether the network is based on free recall or recognition, with correlations ranging between .89 and .93 .

However, there may be advantages to free recall-based networks. Hlebec and Ferligoj examined the relationship between naturally occurring mood and personal networks constructed by free recall or recognition (2001). The authors interviewed students from four college classes about their mood and asked students to list friends and acquaintances by either free recall or recognition from a list of students (the method was fixed for each class. All students in two classes used free recall, and all students in the other two classes used recognition). The authors returned one week later and repeated the method.

They found that, generally, when students' moods were different than before, there was greater instability in personal network size, relative to students whose moods were fairly similar to the week prior. However, the degree of instability differed by how 
Finding The Missing Links, 222

the networks were constructed: when networks were constructed using free recall, personal network size was mostly unaffected by mood. Most of the differences in network size due to mood were observed in networks constructed by recognition. The authors attributed this finding to being because freely recalled ties were stronger (a finding supported by Brewer \& Webster, 1999), and less vulnerable to fluctuations in mood. Thus, although freely recalled networks may be smaller and less representative of a participant's social circle, they may also be more reliable than networks based on name recognition.

Observations. Several of the first social networking studies were performed using observations. As mentioned previously, Wellman extended animal observations to children (1926), while Bott systematized Wellman's approach (1928). Observations are rarely used in mapping out entire social networks, but are instead used for specific social interactions, such as the dominance hierarchies of children (Strayer \& Strayer, 1976; Vaughn \& Waters, 1981) and social support obtained by the homeless (Reitzes, Crimmins, Yarbrough, \& Parker, 2011). Observations are typically carried out by selecting a focal individual and examining the interactions that person has with others. Then, network connections are evaluated based on the observed frequency of certain, preselected behaviors between the focal individual and others.

Observations offer a sort of secure feeling of accuracy and reliability that cannot be guaranteed by surveys, in which lists may be affected by whether they involve free recall of network members or recognition of names from a list (Brewer, 2000) and by participant mood (Hlebec \& Ferligoj, 2001). However, observations are very time 
Finding The Missing Links, 223

consuming to carry out and analyze, and observers must be trained for a long period of time to ensure an acceptable level of consistency. Furthermore, observers may only draw conclusions from what they can see in the environments they have access to. In other words, social networks derived from observations will only be public interaction networks, which may not be what the researchers are actually interested in. Finally, observers must observe interaction periods long enough to gain a reliable record of the interaction patterns. Observing for too short a period can result in conclusions being drawn on atypical interaction data, and observing for too long may wear out the researchers' resources.

Archives. Social networks based on archival data are similar to social networks derived from observations, in that some criteria for a link between entities is determined ahead of time, and then searched for in previously collected data, news reports, or historical documents. Such an approach is obviously not preferred, as it renders the researcher dependent on only the information that was already gathered to make decisions on. However, sometimes the information can be obtained by sources the researcher would likely not have had access to, such as court documents revealing social network information on heavy electrical equipment companies engaging in illegal activity (Baker \& Faulkner, 1993) or information on how social networks contribute to breakthrough roles for Hollywood employees (Skilton, 2008). They are also necessary to construct social networks for populations in the past, such as seventeenth-century villages in the Ottoman Empire (Barkey \& Van Rossem, 1997). 
Finding The Missing Links, 224

\section{Types of Relationships}

In social networks studies, friends are typically used as the relationship that ties two actors together (Keßler, 2010), however, there are plenty of types of relationships to base a network tie on, such as friend, kin, coworker, fellow student, etc. Hanneman and

Riddle note that a social network should not be regarded as a map of all relations between all actors, but just a map of a select one or few relations: "When we collect social network data about certain kinds of relations among actors we are, in a sense, sampling from a population of possible relations" (2005; p. 10). This is an important point, as characteristics of the network have been shown to differ depending on the type of tie the network was based on.

Network structure was found to differ amongst three networks made up by the users of a website called "Essembly" (Hogg, Wilkinson, Szabo, \& Brzozowski, 2008). In this website, users post controversial issues (e.g., "Overall, free trade is good for American workers") and invite others to agree or disagree and engage in debate. The users were members of three networks, as defined by the type of relationship: a social network of people they personally knew (identified by users as "friends"), an ideological network of like-minded individuals ("allies"), and a network of people they disagreed with ("nemeses"). The researchers analyzed the structure of each network and found significant differences: friends were the most modular at $73 \%$ (indicating that the friends network contained the most intragroup connections, as there were $73 \%$ more connections amongst members of small networks than if all ties had been distributed at random), allies had the shortest average path length of 3.77 (indicating that, on average, two users 
Finding The Missing Links, 225

were separated by 3.77 paths), and nemeses had the highest density of network connections at $0.3 \%$ (indicating that $0.3 \%$ of all possible ties between network members were present, that the network was the most densely connected of the three. Thus, people tended to disagree with each other more often than they agreed).

Furthermore, the effect of ties between network members was found to differ by the relationship between members. Roberts and Dunbar found that the costs of maintaining kin relationships was lower than the costs of maintaining friendships, reflected by the finding that the time of last contact was greater for kin than for friends, suggesting that more frequent contact was necessary to prevent friendships from eroding than kinships (2011). In addition, in a study of political activist recruitment, Lim found that the type of relationship was more important for recruiting protest participants than the strength of the network tie, with the most influential relationships being those formed in civic associations, and not the closest relationships (2008). Finally, an investigation into eating behaviors revealed that level of similarity in such behaviors differed by the type of relationship (among spouses, friends, brothers and sisters), with spouses displaying the most concordance (Pachucki, Jacques, \& Christakis, 2011). Such findings are no doubt be important for studies of homophily.

In light of these findings, researchers would be advised to consider measuring multiple relationships and generating networks based on each. Insights revealed from differences between these networks may yield a more rich understanding of the phenomena under study. At the very least, researchers should carefully consider which type of relationship is most meaningful for the study. 
Finding The Missing Links, 226

\section{APPENDIX C: Other Network Identification Methods}

\section{Sociometry}

Sociometric methods enjoy popularity amongst researchers who investigate the effects of people who occupy the fringes of their social environments (whether they are isolated, disliked, or very popular). In sociometric studies, at their most basic, actors are asked to indicate other actors they have a personal preference to be with or away from (liking or disliking). These need not be based on personal liking, any shared activity is open to study (i.e., who one wishes to co-author a paper with). The nominations are collapsed across all respondents to calculate individual actors' Social Preference scores (whether an actor is generally liked, disliked, or neither) and Social Impact scores (how salient an actor is to the group under study). In this section, I will briefly discuss the development of the sociometric method, three of the most common approaches (standard scores, probabilities, and peer ratings), reliability and validity, and limitations to the method.

Methods. Sociometry was developed by Jacob Moreno (1951) as a way of overcoming the problem of a deep discrepancy between the public and private behavior of participants of social arrangements, inherent in observational research. In school research, researchers initially only asked students of other students in their class they preferred to be with, but not those they preferred to avoid, due to ethical concerns that certain "unpopular" students would experience greater animosity from classmates after the study (Gronlund, 1959 in Newcomb, Bukowski, \& Pattee, 1993; Northway, 1946). Other researchers found that relying only on ratings of liking confounded students who 
Finding The Missing Links, 227

were disliked and students who were merely ignored, so they solicited nominations of dislike as well (Lemann \& Solomon, 1951; Thompson \& Powell, 1951).

This concern of increased animosity has been investigated. Hayvren and Hymel (1984) observed classmate interactions in two preschool classrooms in the weeks before and after a survey of children's most- and least-preferred playmates. They found that, in the weeks after the survey, students tended to have significantly more positive interactions with others they liked, but their frequency of negative interactions with students they did not like were not significantly changed. Iverson \& Iverson (1996) similarly performed a sociometric study on a set of fifth-grade children and interviewed 45 of them after summer vacation. One third of the now sixth-graders had discussed their nominations with others (despite being told not to) but did not report treating or having been treated any differently after the study. Thus, research tentatively does not support fears that soliciting negative nominations will lead to poor treatment of unliked children.

The aggregate liking and disliking scores were eventually used to classify the social desirability of students into a high-status group (those who were mostly liked), a low-status group (those who were mostly disliked), and an average group (Lemann \& Solomon, 1952). Binomial probabilities were calculated for the number of likes and dislike a student received, and only students who received a higher than chance amount of likes were placed in the high-status group, while those who received a higher than chance amount of dislike were placed in the low-status group (the rest were placed in the average group). 
Finding The Missing Links, 228

However, an important element was missing from Lemann \& Solomon's study: a measure of social visibility. Their classification scheme confounded two types of average-status students: those who were equally liked and disliked, and those who went unnoticed and accumulated no likes or dislikes. Dunnington (1957) added the number of likes and dislikes for each student together to form a social visibility score, and Peery (1979) had the innovation to place social visibility and social preference in a twodimensional framework.

Finally, Coie and colleagues (1982) developed the system that enjoys wide use today. This system, known as the "Standard Score" approach, uses four scores, all standardized by grade level: number of likes, number of dislikes, social impact (number of likes plus the number of dislikes) and social preference (number of likes minus the number of dislikes). Standardized scores on these variables are used to assign a student to one of five categories: popular students have a social preference $z$-score of more than one, a liked $z$-score of more than zero, and a disliked $z$-score of less than zero. Rejected students had a social preference $z$-score of less than negative one, a liked $z$-score of less than zero, and a disliked $z$-score of more than zero. Neglected students had an impact $z$ score of less than negative one, and liked and disliked $z$-scores of less than zero.

Controversial students had an impact $z$-score of greater than one, and liked and disliked scores of greater than zero. Remaining students were classified as "average".

Alternative procedures. The above method was criticized by Newcomb and Bukowski (1983) who charged that, although it allowed for comparability between peer groups, differences in distribution were not accounted for by standardization, and so 
Finding The Missing Links, 229

group assignments may be subject to biases (under-representation in the case of peer groups with little variation and over-representation in the case of peer groups with a great deal of variation).

Their proposed approach was, instead, to assign based on rarity: a "rare number" of nominations was an amount of nominations whose binomial probability of occurring by chance was .05 or lower. Children were then classified into one of five groups based on whether they had a "rare" like and/or dislike score, and a "rare" impact score. Their classification scheme mirrored Coie and colleagues (1982): popular children had a rare like score and a dislike score below the mean, rejected children had a rare disliked score and a like score below the mean, neglected children have a rare low impact score, and controversial children rare liked and/or disliked score (if only one is rare, the other must be greater than average). Remaining children were categorized as average. This method had a disadvantage of being difficult to apply, especially when children were allowed to make unlimited nominations (though with today's computing power that may no longer apply).

Ultimately, there appears to be little difference between either the standard score or probability methods, as they tend to have fairly similar psychometric characteristics. Research by Newcomb and Bukowski, and by Terry and Coie, showed the standard score and probability methods tend to respectively identify $11 \% / 7 \%$ of children as popular, $13 \% / 11 \%$ as rejected, $9 \% / 15 \%$ neglected, $7 \% / 3 \%$ as controversial, and $60 \% / 65 \%$ as average (1983; 1991). 
Finding The Missing Links, 230

An alternative to the nomination procedure was developed by Asher and colleagues (1979). In this procedure, respondents are asked to rank how much they like other members of the social group under study (or would like to engage in a particular activity with). Individuals' scores are then averaged to arrive at a social preference score. Although nomination procedures dominated the literature during the 1980's and 1990's, the use of continuous scores derived from ratings is becoming more prevalent (Jiang \& Cillessen, 2005). This shift is attributed to improvements in computing power (allowing the management of more complex databases and relatively easy use of advanced statistics) and an increased preference of continuous measures in longitudinal studies.

Major findings. Research in sociometry has primarily focused on determining the predictors and consequences of receiving a certain sociometric status. The rejected status has been associated with the highest risk for negative developmental outcomes (Ollendick, Weist, Borden, \& Greene, 1992; Parker \& Asher, 1987), such as peer victimization (Boivin, Hymel, \& Hodges, 2001; Schuster, 2001), poor academic performance (Hatzichristou \& Hopf, 1996), and dropping out of school (Ollendick et al., 1992).

Approximately half of the children who receive a rejected status are aggressive (Dodge, 1983; Newcomb et al., 1993). These children tend to engage in hostile and unprovoked aggression (Coie, Dodge, Terry, \& Wright, 1991), respond aggressively to vague gestures (Feldman \& Dodge, 1987), and endorse use of aggression to resolve conflicts or gain desired objects (Coie et al., 1991). The other half of rejected children do not tend to be aggressive (French, 1998). Instead, these children have sometimes been 
Finding The Missing Links, 231

found to demonstrate more shy or withdrawn behaviors (Cillessen, van Ijzendoom, van Lieshout \& Hartup, 1992; Rubin, LeMare, \& Lollis, 1990) than non-rejected children, and to engage in unusual or abnormal behavior (Bierman, Smoot, \& Aumiller, 1993). Overall, non-aggressive rejected children appear to be at a lower risk for negative social developmental outcomes than aggressive rejected children (Bierman \& Wargo, 1995). Perhaps not surprisingly, popular children are opposite the rejected children in several ways. Peers tend to describe popular children as helpful, considerate, cooperative, and socially outgoing (Coie et al., 1990), a sentiment backed up by observers (Coie \& Kupersmidt, 1983; Newcomb et al., 1993). Some popular children display a mix of prosocial and dominance behaviors (Luther \& McMahon, 1996; Parkhurst \& Hopmeyer, 1998; Rodkin, Farmer, Pearl, \& Van Acker, 2000) suggesting two subtypes: one of primarily prosocial children, and another of prosocial and dominant/assertive children. Popular children are at a lower risk of internalizing symptoms (Sandstrom \& Cillessen, 2006) than non-popular children, and are more likely to have more adaptive interactions with friends (Allen, Porter, McFarland, Marsh, \& McElhaney, 2005).

The previous two types of children have received the bulk of the empirical attention. With the exception of being low in visibility (by definition), neglected children have proven difficult to find any consistent trends for, other than the fact that they are hard to find consistent trends for (Smith \& Brownell, 2003). Peers will sometimes rate neglected children as somewhat less likeable than others and as shy or withdrawn (Ollendick et al., 1992), and that they lack prosocial behavior (Harrist et al., 1997; Hatzichristou \& Hopf, 1996). However, teachers are likely to rate neglected children 
Finding The Missing Links, 232

more positively, viewing them as motivated in school, functioning independently, and demonstrating prosocial classroom behavior (Wentzel \& Asher, 1995). Correspondingly, research indicates that neglected children have GPAs higher than their rejected and controversial peers (Chen, Rubin, \& Li, 1997; Wentzel, 1991).

Finally, controversial children, who are highly visible and liked and disliked by peers, tend to carry characteristics of both rejected and popular children. Controversial children are often rated by their peers as more aggressive and are seen as arrogant or snobby (Hatzichristou \& Hopf, 1996), yet also seem to engage in relatively high rates of prosocial behavior (Newcomb et al., 1993) and are rated as more sociable (Bagwell et al., 2000). They are somewhat less at risk for negative developmental outcomes, especially dropping out of school, than their rejected peers (Newcomb et al., 1993), but in later ages they appear to play a large role in influencing expression of deviant behaviors in others (Bagwell et al., 2000).

Reliability. Continuous measures have shown good reliability, as assessed by test-retest and longitudinal measures. Jiang and Cillessen performed a meta-analysis of 77 studies, comprising 93 samples and 18,339 participants, looking into the short- and long-term stability of four continuous dimensions of sociometric status: acceptance, rejection, social preference and peer ratings (2005). The authors found good test-retest reliability amongst all four dimensions of sociometric status (reliability correlations of $.72, .70, .82$ and .78 for acceptance, rejection, social preference, and liking, respectively) from studies with less than three months between sociometric measures. Longer-term studies showed lower reliabilities than the short-term studies (as is common), but 
Finding The Missing Links, 233

nonetheless fairly impressive effect sizes: $.53, .52, .58, .52$ for the four categories respectively. These are large effect sizes, as referred to by Cohen's (1977) standards (large $r>.40)$.

Limitations. Jiang and Cillessen (2005) also examined the effects of three study characteristics (time between test and retest, average age of participants, and the year the study was published) on effect sizes for each sociometric category. All study characteristics were statistically significantly related to the effect size of each sociometric category, but their relationships often varied by category. The length of the interval between test and retest was negatively associated with effect size for all sociometric categories (such that reliability was highest when there was minimal time between test and retest, which is to be expected). Meanwhile, average age of participants was positively associated with effect size for all categories except liking, where it was negatively related. Finally, the year in which the study was published was negatively related to acceptance and liking, but positively related to rejection and social preference.

Other researchers have examined the effects of the measurement design on findings. Poulin and Dishion measured 664 sixth graders from three middle schools and examined the effects of alphabetized rosters, cross-sex nominations, and out-ofclassroom nominations on sociometric status (2008). They found significant correlations between position on the roster and number of nominations for being liked least $(r=-.31)$ and liked most $(r=-.14)$. Number of nominations for acquaintances and best friendships were not significantly related to roster position. To control for this roster effect, the 
Finding The Missing Links, 234

authors suggested regressing number of nominations on roster position and using the residuals as the students' number of nominations.

In addition, $48 \%$ of "like least" (LL) and 16\% of "like most" (LM) nominations were contributed by other-sex grade mates. Looking at individual students, the correlation between number of nominations from same-sex vs. other-sex grade mates was $\mathrm{r}=.54$ for $\mathrm{LL}$ and $\mathrm{r}=.58 \mathrm{LM}$. The authors noted that these correlations suggest "that nominations received from other-sex grade mates might bring in unique information." (Poulin \& Dishion, 2008, p. 915). The findings were similar for nominations by classroom: the correlation between number of individual nominations for in-class vs. outof-class peers was $r=.39$ for $L L$ and $r=.51$ for $L M$.

Although not limitations of the method per se, these findings give important implications for researchers to consider: special consideration should be paid to the use of rosters. In addition, who participants accept, reject and prefer seems to crystallize with age, but liking seems to become more fluid.

Finally, the significant relationship between year and effect sizes suggests that society and/or research is changing in ways that influences the stability of participants' relationships with one another. Thus, researchers will need to consider and control for the many ways in which social statuses are formed and maintained (such as via cell phones and online social networking). In addition, the implied changes of society also warns that research today may not be directly comparable to research from many years ago.

However, as discussed previously when considering the effects of different types of relationships, sociometry approaches only yield a limited range of relationships to 
Finding The Missing Links, 235

investigate, those of liking, disliking, and salience. Surely, other participant characteristics can be examined.

\section{Social Crowds}

Another way of socially organizing individuals is by the crowds they are associated with. Social crowds consist of members who may or may not be directly connected, but who share certain salient characteristics. Much social crowd research in the United States is carried out in middle and high schools and typically identify students as belonging to social crowds such as jocks, nerds, burnouts, preppies, etc. (although such crowds have also been observed in college settings [Sessa, 2007]). Names of social crowds often reflect what members have in common, such as shared beliefs, ethnicity, preferences for certain activities, or common interests in clothes or music (Brown \& Lohr, 1987; Brown et al., 2008; Sussman et al., 1990; Sussman et al., 2007).

Adolescents have many reasons to associate with a social crowd. On the surface, crowds provide an excellent place to make friends and, at the very least, tend to be pleasant people to be around (Urberg, 1992; Urberg, Degirmencioglu, Tolson, \& Halliday-Scher, 2000). In addition, social crowds build a sense of autonomy in adolescents by helping them develop independence from their parents (Brown \& Lohr, 1986) and by reinforcing appropriate behavior for the world beyond school (Ashmore, Del Boca \& Beebe, 2002), though some crowds may also reinforce inappropriate and dangerous behavior in the form of drug habits (Barber, Eccles, \& Stone, 1994; La Greca, Prinstein, \& Fetter, 2001), dropping out of school (Farmer et al., 2003) and sexual 
Finding The Missing Links, 236

behaviors (Miller, Farrell, Barnes, Melnick, \& Sabo, 2005). For shy youths, it is suggested that association with certain off-putting crowds (such as goths and punks) may be a mechanism to avoid unwanted social interactions (Besic \& Kerr, 2009; the authors found that, although shy youths were successful in avoiding social interactions with strangers, they were not any happier for it).

Looking deeper, crowds may also be integral to adolescent identity development. Newman and Newman (1976) argue that, in terms of Erik Erikson's theory of Psychosocial Development, the central crisis of early adolescence (ages 13-17) is one of "group identity vs. alienation." The authors explain that, first, adolescents must identity with a social crowd, and Newman and Newman predict that this crowd must be of an acceptable social status for them to develop good self-esteem (a hypothesis which has found support by Brown and Lohr [1986] who determined that the self-esteem of students in middle and high schools was strongly affected by the social status of the crowd they were nominated by peers as belonging to, and that the students with the lowest average self-esteem were those who did not belong to a group at all [though it was higher when they perceived that they belonged to a group]. In addition, La Greca and Harrison [2005] found that depression and social anxiety showed a similar pattern). Once adolescents understand their group identity, they come to understand how they differ from the group, thus developing an individual identity. Newman and Newman (2001) have since extended this theory toward the development of ethnic identity.

Methods. The effects of social crowds must be studied by first identifying the crowds that adolescents belong to. There are three commonly used methods for 
Finding The Missing Links, 237

identifying social crowds (Sussman et al., 2007). The first is self-identification, in which students are simply asked which crowd they feel they belong to. The second is peer nomination, in which students in a school are asked to place each of their peers into a crowd (often from a list designed ahead of time). The third involves the use of ethnographic methods (such as interviews, focus groups and observations) so the researcher herself may decide what the crowds are and who belongs in each.

Sussman and colleagues reviewed 44 studies on the social crowds of middle- and high-school students (2007). Of those studies, 39 used one of the three main methods (the other five surveyed students and used various statistical procedures to separate students into crowds based on similar characteristics). The authors found that self-identification was by far the most commonly used method, with 24 of the studies employing it. Although it is certainly the easiest of the three methods to use, this is rather surprising, given that an obvious drawback to this method is that social-desirability biases might tamper with results (especially given the advantages of associating oneself with a highstatus group). In addition, Brown, Mory, and Kinney (1994) argue that the social crowd is defined by stereotypes attributed by others. If they are correct, it would seem that students' peers would be the only people knowledgeable about social crowds. In this review, eight papers used peer-nominations.

However, peer report should not be viewed as an unfailing measurement (Urberg et al., 2000). First, peers must know of the crowds in the school, the relevant stereotypes, and other students. Gaps in their knowledge will be reflected in the data. In addition, each student interprets and ascribes stereotypes differently, which affects the crowd they will 
Finding The Missing Links, 238

assign a student to. For instance, Urberg gives an example in which Burnouts may view Preps as stuck-up, but Jocks may view Preps as friendly. Ultimately, there will be a limit in the level of consensus attainable. Finally, some social crowds tend to be socially close together (such as the aforementioned Jocks and Preps), while other crowds tend to be socially distant (such as Goths and Punks). As such, students may only be familiar with their crowd and related crowds, and able to give an accurate assessment, but unfamiliar with more distant crowds. They may incorrectly assign students or lump all socially distant crowds together.

Urberg and colleagues (2007) investigated the concordance of self- versus peernominations for social crowds and found a reasonable degree of overlap (66\%), which was stronger for females and older adolescents. In addition, relations of crowds to various behaviors did not vary between self- and peer-nominations. Thus, the authors recommended either measure could be used for many purposes. Given the aforementioned concerns, more research will nonetheless be needed to determine the finer details of either method.

Finally, the ethnographic methods, which were used in seven of the 44 studies reviewed have the advantage of a lack of bias (or, at least, a perceived lack of bias) and the ability to uncover a wealth of deep information. However, they involve the most work by far, and the researcher must be careful to learn everything the students have to know about the social crowds at their school: what crowds there are, what the stereotypes are for each one, and which students these stereotypes apply to. As is common in social 
Finding The Missing Links, 239

science research, a combination of procedures will probably turn out to be the most accurate approach.

Major findings. The review by Sussman and colleagues (2007) revealed that five general categories of social crowds were found in most social crowd research, regardless of the method used, what the authors called the "Elites", "Athletes", "Deviants", "Academics", and "Others". It should be noted that these are not specific crowds researchers have found in schools, but are instead general categories. For example, Deviants can include social crowds such as goths, punks, burnouts, stoners, toughs, etc. Nonetheless, crowds within these general categories tend to have several common characteristics.

According to the authors, Elites tend to be high in peer status (i.e. popular) and social involvement, and are somewhat involved in academics. They are somewhat likely to engage in alcohol and risky sexual behaviors. In the review, they were described in 34 of the 44 studies.

Athletes were similar to Elites, in that they were generally high in peer status and social involvement, however they were lightly involved in academics. In studies of social crowds, Elites and Athletes were often confounded or merged together, possibly due to their similar social characteristics. Nonetheless, Athletes were described in 33 of the reviewed studies.

Deviants were the most consistently found crowd, with 37 of the studies describing them (including all three of the studies not conducted in the U.S.). Deviants tended to be moderate in their peer status and social involvement, and they were often 
Finding The Missing Links, 240

opposed to academics. A multitude of studies consistently found them to be most likely to use tobacco, alcohol and drugs, and engage in risky sexual and violent behaviors, when compared with any other cluster of social crowds. Studies of life-satisfaction amongst this group have sometimes found Deviants to be the lowest in life-satisfaction, and sometimes a different group is. However, in no study was the life-satisfaction of Deviants ever high.

Academics, which were described in 27 studies, were in the middle of peer status, low on social involvement, and tended to be highly involved in academics. They were most likely to graduate college by age 24 .

The last category was Others. This was essentially a catch-all category for those students who were otherwise uncategorized, and were often given a plurality of names in other studies such as normals, averages, regulars, nobodies, outsiders, loners, nerds, outcasts and floaters. Generally, Others were low in peer status, social involvement and academics. In some studies they were thought of as average students who did not stand out from the group enough to be categorized into a specific crowd, while in others they were viewed as students so disengaged from the social scene they did not associate with a group. Others were described in 41 studies.

Limitations. In Sussman and colleagues' review (2007), a widespread problem in the design of the studies was noted: generally, researchers were interested in a certain social crowd, and so they set out to determine which students were in that crowd and which were not. Thus, their study generally compared the characteristics of a single, welldefined social crowd (often Deviants) with another crowd of everyone else, which the 
Finding The Missing Links, 241

authors dubbed the "Others". Found that 41 of the 44 studies described, the characteristics of Others always changed from study to study and they were generally given a plurality of names like such as normals, averages, regulars, nobodies, outsiders, loners, nerds, outcasts and floaters. Adding to the confusion, researchers of some studies thought of them as average students who did not stand out from the group enough to be categorized into a specific crowd, while others viewed them as students so disengaged from the social scene they did not associate with a group. Thus, this crowd, which is a very common crowd to compare some target crowd to, is often poorly defined and conceptualized, limiting its usefulness.

In addition, in only two of the 44 studies were students allowed to select more than one social crowd (Kipke, Montgomery, Simon, Unger, \& Johnson, 1997; Kipke, Unger, O’Connor, Palmer, \& LaFrance, 1997). As it is likely that students identify either themselves or classmates as belonging to at least two social crowds (for example, an Academic Deviant or an Elite Athlete), the secondary and tertiary crowds that students perceive themselves and others as belonging to should be measured and controlled for, to improve the power of analyses and clarify the characteristics of the social crowds. Although much expanded from the sociometry method, social crowds still have some shortcomings to the social networks researcher, as they fail to yield any sense of the structure of the population under study. Who knows who? Which groups are influential? What is the effect of group size on individual outcome? 
Finding The Missing Links, 242

\section{APPENDIX D: Preschoolers' Friendships}

The friendships of preschoolers and young children often start out as being based around shared activities (Bigelow, 1977) with children engaging in parallel play, then imitation of one another (Hanna \& Meltzoff, 1993), and finally progressing to cooperative play (Urberg \& Kaplan, 1989). From here, preschool friends tend to fall into one of three friendship types (Kerns, 2000). The first involves friends who are harmonious but relatively independent during play, while the second involves friends with low levels of harmony or interactive play. The third and most common involves pairs of friends who are harmonious, responsive, and interactive during their play. Indeed, recreation seems to become the basis for the friendship's identity, as preschoolers often define their friendships around play (Bigelow, 1977; Selman, 1980). When conflicts erupt between friends (which happen no less often than between non-friends), they tend to be shorter-lived, less intense, and with more equitable outcomes than among nonfriends (Hartup et al., 1988).

Male and female preschoolers prefer playmates who are physically attractive, intelligent, emotionally stable, have a positive personal self-concept, and have a positive social self-concept (Drewry \& Clark, 1985). Personal similarity is important, but sometimes in unexpected ways. Fawcett and Markson (2010) found that preschoolers showed an attraction to dolls based on similarity in hair color, but not shirt color, and in similarity in behaviors, both relevant (such as toy preference) and irrelevant (such as food preference) for play. 
Finding The Missing Links, 243

Gender and race are commonly studied variables in young children's friendship preferences. Toddlers tend to show same-gender preferences, with such preferences occurring earlier in girls (LaFreniere et al., 1984). However, cross-gender friendships are not uncommon, with one study showing one third of preschool peer groups containing males and females (Smith \& Inder, 1993). When cross-gender friendships are found, girls are observed to be more liked and more likely to be chosen by boys than boys are to be chosen by girls (Djon \& Berscheid, 1974; Hayes, 1978). Although early explanations for this divide focused on differences in boys' and girls' behaviors, playstyles, and interests (Eisenberg, Tyron, \& Cameron, 1984; Moller \& Serbin, 1996; Serbin, Moller, Gulko, Powlishta, \& Colburne, 1994), this does not account for the gender-segregation observed in activities common to both genders, such as the sandbox, puzzles, and on the swings (Maccoby \& Jacklin, 1987). More recently, preschooler social cognitions of gender that often conceptualize a cross-gender friendship as incompatible or abnormal have been shown to account for some of the preference (Martin, Fabes, Evans, \& Wyman, 1999).

Race is generally viewed as a less prominent factor in friendships than gender. In Kinzler and Spelke's (2011) study of social relations and race amongst young children, they found that 10-month-old infants were equally likely to steal a toy from an other-race infant as they were a same-race. Furthermore, 2.5-year-old children showed no same/other racial preferences in giving toys to other children. However, by age 5, children verbally expressed clear preferences for same-race individuals. Part of this finding may be due to an early lack of awareness of race in social reasoning, which does not begin to emerge until around four years of age (Shutts, Roben, \& Spelke, 2013). 
Finding The Missing Links, 244

Ultimately, when racial preferences emerged, they tended to be associated with a preference of one's own race, and not so much rejection of other races (Kowalski, 2003).

In addition, it has been demonstrated that at least some of this racial preference may actually be due to unfamiliarity with a particular accent, and not specifically race (Kinzler, Shutts, DeJesus, \& Spelke, 2009). 
Finding The Missing Links, 245

\section{APPENDIX E: Adolescent Friendships}

Amongst adolescents, friendships continue to provide social integration, feelings

of worth, and, to a lesser extent, emotional intimacy (Furman \& Buhrmester, 1992). In addition, when family support is lacking or the adolescent lives in a stressful environment, coping is aided by friends (when such friends are strong and protective). This effect can be observed with even a single friend (Hodges, Boivin, Vitaro, \& Bukowski, 1999). Indeed, adolescent adjustment and performance is related to both the amount and quality of friends. Friendships also become far more important, as the amount of time spent with friends increases substantially, along with a subsequent drop in the amount of time spent with family (Larson \& Richards, 2001). Friendships also become more emotionally intimate, causing social crowds (which once virtually controlled who a child could and could not be with) to lose much of their importance and hierarchy, and to become more permeable (Gavin \& Furman, 1989). The strength with which friends become such an important part of an adolescent's life has led to some indications that adolescence may well be a sensitive period for sociocultural processing (Blakemore \& Mills, 2014). Ultimately, the increased closeness and time spent with friends will set the stage for a number of enduring social consequences, including the beginnings of romantic relationships and identity development.

\section{Romantic Relationships}

Adolescence is an important period for the development of romantic relationships. These special relationships seem to have come into their own among researchers in the 
Finding The Missing Links, 246

past few years (Collins, 2003), after decades of academic neglect due to a common perception that adolescent relationships were transitive and superficial (Brown, Feiring, \& Furman, 1999). More recent research has shown that adolescent relationships are far from transitive, with $20 \%$ of 14 -year-olds reporting having been in a relationship for at least 11 months, $35 \%$ of $15-16$-year-olds, and around $60 \%$ of $17-18$-year-olds in one national study (Carver, Joyner, \& Udry, 2003). Adolescent relationships are also far from superficial and unimportant, with adolescents describing romantic relationships in terms similar to how adult romantic relationships are often described (Levesque, 1993; Connolly, Craig, Goldberg, \& Pepler, 1999). Furthermore, researchers have found that being in a relationship is associated with several developmentally-relevant events (for an in-depth review see Furman \& Shaffer, 2003). On the beneficial side, having a romantic relationship (especially a high quality one) is associated positively with romantic selfconcept and, in turn, feelings of self-worth (Connolly \& Konarski, 1994; Harter, 1999; Kuttler, La Greca, \& Prinstein, 1999). Furthermore, longitudinal research has demonstrated that self-perceived competence in romantic relationships emerges as a consistent element of general competence (Masten et al., 1995). However, compared to unattached adolescents, those in romantic relationships report experiencing more conflict (Laursen, 1995), and demonstrate more intense mood swings than are more normal for this period of development (Larson, Csikszentmihalyi, \& Graef, 1980; Larson \& Richards, 1994; Savin-Williams, 1996). Joyner and Udry (2000), in examining data from a national study of adolescents, even found that adolescents in romantic relationships scored between 10-20\% higher on the CES-D depression scale. However, Collins (2003) 
Finding The Missing Links, 247

suggested that break-ups may be more to blame for the link between adolescent romantic relationships and depression. In line with this sentiment, romantic break-ups are the single most common trigger for the first episode of a major depressive disorder (Monroe, Rhode, Seeley, \& Lewinsohn, 1999). Thus, romantic relationships seem to be neither momentary nor insignificant, rather they are major life events associated with real benefits and real challenges, and their ending is unlikely to be of any minor concern. Their beginnings lie in the participants' peer networks.

As previously noted, adolescents spend increasing amounts of time with their friends, and less so with their families. This increased time with friends gradually progresses to cross-gender friendships at around the time adolescents normally begin dating (Richards, Crowe, Larson, \& Swarr, 1998), and, ultimately, to dyadic romantic relationships (Connolly et al., 2004; Feiring, 1999). Thus, romantic relationships most commonly begin in the context of a large, loosely connected group of friends pairing off with one another (Connolly, Furman, \& Konarski, 2000).

Before attainment of a romantic partner, friends are influential in a number of ways. These include people with which to discuss sexual issues (Dilorio, Kelley, \& Hockenberry-Eaton, 1999), social support during the pursuit of a romantic partner (Connolly \& Goldberg, 1999), a place to build one's social competence (which is ultimately associated with attainment of a romantic partner; Furman, Ho, \& Low, 2008), an emotionally intimate context with which to experience and manage emotions (Connolly et al., 2004), and models for one's romantic partner (Connolly \& Goldberg, 
Finding The Missing Links, 248

1999) as well as for interactions in a romantic relationship (Furman \& Shomaker, 2008;

Furman, Simon, Shaffer, \& Bouchey, 2002).

Once in a romantic relationship, adolescents tend to shift importance from their friends to their partner (Dunphy, 1963; Johnson \& Leslie, 1982; Sullivan, 1952), typically by spending less time with them (Zimmer-Gemeck, 1999), although they do not normally eliminate their friends entirely (Richards et al., 1998). In addition, the degree of companionship the attached adolescent derives from their friendships decreases (Barry, Madsen, Nelson, Carrol, \& Badger, 2009). Despite their reduced status, friends still provide social support and assistance during periods of difficulty (Connolly \& Goldberg, 1999), and qualities of their friendship influences qualities of the romantic relationship (Collins \& Van Dulmen, 2006; Furman et al., 2002), as well as when the adolescent's first sexual intercourse occurs (Sieving, Eisenberg, Pettingell, \& Skay, 2006).

\section{Identity Development}

Erik Erikson argued that ego-identity development is the central developmental task of adolescence $(1963,1968)$, a view which has come to be widely accepted. Marcia operationalized Erikson's considerations about adolescent identity by developing four statuses with which to classify one's identity, based on one's past and present active exploration of life's alternatives as well as one's level of commitment to their personal values (Adams \& Montemayor, 1983; Marcia, 1966). The four statuses, which may be proceeded through in any particular order, skipped, or revisited, are: 1) Identity Achievement, in which the person has formed commitments of their own after a period of 
Finding The Missing Links, 249

exploration; 2) Moratorium, in which the person is exploring and has made no commitments; 3 ) Foreclosure, in which commitments are formed without an adequate level of exploration; and 4) Diffusion, in which very little exploration has been conducted and the person has few, if any, commitments. Marcia's model is not without its criticisms (see Miller, 2010), but he has provided a fairly well-supported paradigm around which to organize study.

Research has indicated that friends play an important role in the adolescent's identity development. Having strong interpersonal relationships, including friendships, is associated with identity achievement (Archer \& Waterman, 1983; Cooper \& Grotevant, 1987). Furthermore, mutually identified same-sex friends were significantly more similar in many aspects of identity than non-mutual or randomly paired adolescents (Akers, Jones, \& Coyl, 1998). Specifically, mutual friends were significantly more similar on global measures of foreclosure and diffusion, though not on achievement or moratorium. Within the content areas of dating, education, friendship, lifestyle, and occupation, mutual best friends were highly significantly more similar for all four identity statuses (in other words, 20 pairwise comparisons, consisting of comparisons of the four identity statuses over five content areas, were highly significant $[\mathrm{p}<.001$ for each case $])$. Furthermore, mutual friends were generally more similar on interpersonal identity areas than ideological, which was suggested to be because "adolescents tend to devote greater personal effort toward exploration and resolution of interpersonal issues than toward ideological issues" (Akers et al., 1998, p. 195). 
Finding The Missing Links, 250

Friendship conflict and support have been found to impact adolescent identity development. Reis and Youniss found that persistent conflicts with friends were associated with losses in identity (2004), while, Jones and colleagues demonstrated that friendship conflict was positively associated with moratorium and diffusion, and support negatively associated with diffusion (Jones, Vaterlaus, Jackson, \& Morrill, 2014).

According to Azmitia, "conflicts were shown to be of increasing importance throughout adolescence, as unsolved conflicts are associated with deterioration of the self' (2002, p. 174).

Besides through friendship features, friends themselves influence one another's identity development. Josselson indicated that adolescents can experiment with various ways of being with encouragement and support from friends and peers (1996), and that a sense of self is validated in what is reflected back by others (1992). Meanwhile, Kerpelman and Pittman suggested that identity exploration may be initiated by feedback from peers regarding who they could be in the future (2001). Several mechanisms by which friends influence one another's identity development have been suggested.

In their qualitative study of first-year Japanese university students, Sugimura and Shimizu determined that students influence one another's identity development through five processes (2010). The first was identity concern, or the provision of sympathy and confirmation for others. They found that adults could provide this too, but it was likely to be deeper and more helpful coming from others in the same situations. The second were identity goals, providing goals to aspire toward. Identity clues were the third process, or the provision of ideas that a friend could use in their identity development. The fourth 
Finding The Missing Links, 251

was companionship and the enthusiasm shared by two friends exploring the same ideas together. The final process was bridging, in which friends help one another translate ideologies into more practical forms than can be used in the world at large.

Another suggested mechanism concerns the stories friends tell one another. Adolescents increasingly tell one another stories from their lives as they grow older (McLean, 2005), which serve functions to make sense of one's past, present, and their projected future (McAdams, 1993; 2001). In her study, McLean (2005) found that some adolescents told stories simply for entertainment value, but she noted that this may well represent a different kind of meaning, a sort of meaning oriented around the valued audience for one's stories.

Friendship also has an indirect route by which it may influence identity development. In one study, identity achievement was associated with several features of romantic relationships (companionship, worth, affection, and emotional support), but was associated with nothing for friendships (Barry et al., 2009). The authors suggested that, due to the disengagement of attached adolescents from their social circles, friends may lose some of their influence simply due to distance, with many of their functions being picked up by the romantic partner. These findings certainly follow other researchers who argue that progress toward a mature identity and mature intimacy happen simultaneously (Paul \& White, 1990; Seginer \& Noyman, 2005). Thus, an indirect path is implied by which friends may influence identity development through friends helping one another into romantic relationships and influencing their progression. As best as can be determined, there has been no research into the identity development of the adolescents 
Finding The Missing Links, 252

who are not in long-term relationships (40\% of 17-18 year-olds [Carver et al., 2003]), where it would seem likely that friends would have more influence over one another. 
Finding The Missing Links, 253

\section{APPENDIX F: Unilateral Friendships}

In the long history of friendship research, friends have almost always been conceived as mutual and balanced (Carley \& Krackhardt, 1996; Rubin, Bukowski, Parker, \& Bowker, 2008). Unilateral nominations, then, are something of a "forbidden zone" in this field. This is curious because, for one thing, they are commonly found. Studies of preschoolers show that about 80 and $90 \%$ of all children have at least one unilateral friend (Gershman \& Hayes, 1983; Gleason \& Hohmann, 2006; Vaughn et al., 2000), while studies of school-aged children demonstrate similar levels, at between 80 (Guay et al., 1999) and 85\% (George \& Hartmann, 1996). However, researchers often do not know what to make of them. Did one of the respondents simply forget to name one of their friends? Is this a case of wishful thinking? Does this represent a sort of unbalanced friendship (Berndt, 2002)? Interestingly, despite that researchers tend not to consider unilaterally nominated friends as "true friends", they do not generally combine these dyads in with non-friends either, typically opting to completely ignore them (Baines \& Blatchford, 2009; Koster, Pijl, Nakken, \& Van Houten, 2010; Parkers, Kovacs, \& Hoffman, 1996; Schneider, Fonzi, Tani, \& Tomada, 1997), a move that may lose a representative picture of friendship in school (Gleason \& Hohmann, 2006).

There is some evidence to view unilateral friends as something between nonfriends and mutual friends. On the one hand, unilateral friends are liked significantly more than non-friends by both the nominee and the recipient (Hundley \& Cohen, 1999), and have their own reasons for liking them over non-friends (Gershman \& Hayes, 1983). During times of conflict, although unilateral friends will tend to feud in a manner more 
Finding The Missing Links, 254

similar to non-friends than mutual friends (with less concern for a balanced outcome), unilateral friends often will continue to socialize with one another afterwards, unlike with non-friends (Hartup et al., 1988).

Nonetheless, children seem to show an understanding that their unilateral friendships differ from their reciprocal ones. Children like their reciprocal friends the most (Hundley \& Cohen, 1999) and for different reasons than for unilateral friends (Gershman \& Hayes, 1983; Hayes, Gershman, \& Bolin, 1980;). When asked to represent their reciprocal and unilateral friendships as drawings, children in reciprocal friendships generally depict themselves as more similar and cohesive with their reciprocal friends, and with a better understanding of their partner's emotions (Laghi et al., 2014). Reciprocal friendships tend to be more stable over both a six-week period (Clark \& Drewry, 1985) and over the course of a school year (Gershman \& Hayes, 1983).

Thus, a unilateral association appears to be a distinct kind of relationship, not on the same level as "true" mutual friends, but more than non-friends. The friendship literature would do best to reconsider its stance on unilateral relationships, as they do not represent a mere curiosity, but may well be an important source of influence. Despite that some researchers intentionally exclude unilateral friendships from studies of influence (e.g., Vitaro, Brendgen, \& Tremblay, 2000), researchers have found evidence of influence between unilateral friends on measures of deviant behavior (Werner \& Crick, 2004) and on competence and motivation (Altermatt \& Pomerantz, 2003). Although such influences tend to be stronger for reciprocal friends than for unilateral friends, the existence of an influence at all is worth noting, especially for the fact that children tend to 
Finding The Missing Links, 255

have more unilateral than reciprocal friends (thus, the amount of influencers may compensate for their individual moderate strength). Researchers have yet to consider the influence of the entire group of unilateral friends as a whole, which may well yield an influence worth taking note of. 
Finding The Missing Links, 256

\section{APPENDIX G: Methods Comparison}

Due to the diversity in social networks research methods, selecting the most appropriate one can be a daunting task. As such, comparisons have been performed of different variations of the methods, examining similarities and differences in the resulting social networks. As sociometric ratings and social crowds are conceptually distinct from friendships, comparisons tend to embrace differences between the methods as sources of rich data. On the other hand, peer nominated social networks are often conceptualized as an alternative to self-reported friendships, even if that goes against Cairns and colleagues' original intention $(1985 ; 1998)$, and are viewed as a source of much of the same information. As such, comparisons of the methods tend to interpret differences between the methods as inaccuracy on the part of peer-nominated measures. The prominence of self-reported friendships is also reflected by the comparisons: every single comparison that was found compared the social network methodology of choice (sociometric ratings, social crowds, or peer-nominated networks) with self-reported friendships. This section will begin with a brief review of these comparisons, then there will be a discussion of the potential inaccuracy of this attitude and what peer-nominated data may be able to report on that the others cannot.

\section{Sociometry}

Comparisons of friendships and sociometric data have generally shown a positive relationship with being liked having friends. Erdley and colleagues (1998) compared agreement rates between reciprocal nominations and various combinations of sociometric 
Finding The Missing Links, 257

ratings and unilateral nominations encountered in the literature. She found that, out of a total of 90 students across three fourth- and two fifth-grade classrooms, only 60 pairs of reciprocal nominations were found. However, when compared with a unilateral nomination from one student and a liking score of five out of five from the other student (a criteria which was met by 84 pairs of students), the two methods showed a high level of agreement according to kappa statistics $(\kappa=.80)$. Agreement rates were much lower when reciprocal nominations were compared with unilateral nominations and either mutual liking scores of four or five $(\kappa=.67)$ or unilateral nominations and mutual average liking scores of at least four $(\kappa=.60)$. Removing unilateral nominations and simply comparing reciprocal nominations with mutual liking scores of four of five, agreement was much lower $(\kappa=.52)$.

Similarly, Gest, Graham-Bermann, and Hartup (2001) found a highly significant correlation of $r=.46$ with being liked most and the number of one's friends (the correlation between being liked least and number of friends was $r=-.20$, still significant at $p<.01)$. In addition, in examining sociometric statuses, the authors found that popular students were also most likely to have at least one reciprocated friendship (69\%) followed closely behind controversial students (64\%). Just over half of average students had at least one reciprocated friendship (55\%). Only about one third of rejected children had friends (39\%), while neglected children were the lowest with nearly half as many $(21 \%)$.

Thus, both studies indicate a relationship between being liked in general and having friends. However, unpopular children still have friends, and friends do not always 
Finding The Missing Links, 258

like each other perfectly (nor do pairs who like each other perfectly consider each other to be friends).

\section{Social Crowds}

Urberg examined the self-reported social crowds and reciprocally nominated friendships of all eleventh grade students at a high school (1992). She found that, of the three social crowds in her study (Preps, averages, and burnouts), reciprocally nominated friendships came from within the student's crowd a majority of the time $(51.5 \%$ for burnouts, $60 \%$ for preps, and $70 \%$ for averages). Preps and burnouts also had friends from the averages about one third of the time (33.3\% and $37.2 \%$, respectively), and rarely ever from each other $(6.7 \%$ of preps had a friend who saw themselves as a burnout, while $11.3 \%$ of burnouts had a friend who saw themselves as a prep). Averages were evenly split in whether friends saw themselves as preps $(14.3 \%)$ or burnouts $(15.7 \%)$. In a follow-up study involving two schools, Urburg and colleagues (1995) found a smaller proportion of in-crowd friendships, at just $21.8 \%$ and $33.6 \%$. This apparent discrepancy may be due to the higher amount of social crowds found in both schools (six and eight, respectively).

Finally, in another study of middle- and high-school social crowds, Urburg and colleagues (2000) found that, while members of all seven crowds had friends from within their own crowd at a rate that was higher than chance (and that friends were most likely to come from within the crowd), members of crowds were often friendly with members other crowds, with all but one crowd (jocks) having friends from between one to three other crowds. The most self-interested crowd were burnouts, who were 10.5 times more 
Finding The Missing Links, 259

likely than chance to have same-crowd friends, while the least were averages, whose same-crowd friend rate did not differ from chance. A comparison of the crowd sizes and rate of same-crowd friendship revealed a Pearson's correlation of $r=-.62$ (which leaps to $r=-.92$ if burnouts are excluded as outliers).

These studies indicate that, although same-crowd friendships should be expected, friendships are not exclusive to the crowd a person is part of. In addition, the inverse relationship between social crowd size and rate of same-crowd friendship falls in line with other research on homophily (see the homophily section in the next unit for more details), indicating that people are more likely to seek out others like them when few others are like them. 
Finding The Missing Links, 260

APPENDIX H: Controlling for Network Dependencies

Three broad strategies tend to be used in controlling for network dependencies. First, network structure can be incorporated via covariates. For example, in Gulati and Gargiulo's (1999) study of interorganizational networks, analyses consisted of multiple regressions which included covariates representing individual firms' centrality, network density, interdependence, etc. This strategy suffers from the assumption that the observations are otherwise independent of one another and, as such, any hypothesis testing is bound to give incorrect standard errors (Proctor, 1969; Laumann \& Pappi, 1976). Although it certainly served its purpose before better methods were developed, this strategy is not considered to be suitable anymore.

A second approach is to control for certain aspects of network dependencies, though without modeling them explicitly. Among the most common versions of this approach is a permutational procedure developed by Krackhardt (1987) adapted from the Quadratic Assignment Procedure (QAP). In this version, actors are randomly rearranged in the network while maintaining the pattern of ties (permuted) and they form a sort of "null”, randomly designed network. Each possible permutation of networks is calculated and multiple regressions are carried out for them, forming a distribution of correlations, against which the observed correlation can be compared. From here, significance can be inferred by an observed correlation's percentile placement along the distribution of correlations from the randomly generated networks. As the number of permutations is determined by a factorial $(\mathrm{N} !)$, the amount of networks can quickly become unfeasible with even a modest amount of actors. However, the mean and standard deviation of the 
Finding The Missing Links, 261

distribution can be calculated based on the cell values of the social network matrices (Mantel, 1967; Hubert \& Schultz, 1976) and the significance level of the observed correlation can be determined by the appropriate region beyond the Z-score under the normal curve (thus, normality of the null correlations is assumed in this approach. A nonparametric approach to an excessively large network could also be to use bootstrapping [see Duval, 1993]).

An alternative to Krackhardt's approach deals with what are known as Conditionally Uniform Models. In this case, the researcher compares their network to other similar networks, and she compares her observed statistics to statistics computed from these other networks that obey the same network dependencies. This approach can be helpful to test relatively complicated structural properties while controlling for a few fairly simple network dependencies, while the QAP approach can be useful when research interest is only on the effects of explanatory variables, and not on network modeling or structure.

The final variation is to specifically model the structural dependencies between tie variables, instead of treating them as nuisances (as the previous approaches did). In this method, a probability model predicting which actors will make ties with one another is formulated by the researcher, and the current network is perceived as a random draw from that model (Snijders, 2011). The model is then used to derive statistical procedures and their properties for hypothesis testing. There are two basic classes of approaches that apply this strategy. 
Finding The Missing Links, 262

The first class of approaches defines each actor as a latent (i.e. unobserved) variable (Lazarsfeld \& Henry, 1968). Consequently, each actor's observed variables have a simple probability distribution, given the latent variables. Models that fall under this approach include the stochastic block model (Daudin, Picard, \& Robin, 2008; Holland, Laskey, \& Leinhardt, 1983), distance models (Freeman, 1992; Hoff, Raftery, \& Handcock, 2002), and directed models (Holland \& Leinhardt, 1981; van Dujin, Snijders, \& Zijlstra, 2004).

In the second class, each network tie is explicitly defined as a random variable, and the model that has been defined to predict the network is used to assign a probability to the existence of each tie (Robins, Pattison, Kalish, \& Lusher, 2007). These probabilities can be compared to the observed network for a sense of the fit of the model, and then parameters can be estimated from there. 
Finding The Missing Links, 263

APPENDIX I: Stochastic Actor-Based Modeling

In this design, mathematically described in Snijders, Steglich, and Schweinberger (2007), an explicit probability model is defined for the joint evolution of a social network and the traits of its inhabitants (Pearson et al., 2010). These models assume changes happen in continuous time, one at a time, at stochastically (randomly, but somewhat predictably) determined moments. Such a model assumes that components are not independent of one another. Thus, network interdependence and incompletely observed data can be accounted for with this design. Furthermore, the freedom afforded by explicitly specifying network evolutionary processes allows for the assessment and controlling of primary mechanisms (such as homophily and influence) and alternative mechanisms (such as structural contextual processes). Finally, this model is agent-based, allowing for the analysis of individual actors on the outcomes of target individuals.

To start, the researcher must specify a list of rules of network change (predictors of a tie between two actors) and of behavior change (predictors of some actor behavior under study, such as substance use). For example, in their study of substance use amongst sport-active adolescents, Pearson and colleagues (2006) specified several processes of network evolution, including outdegree (ties sent from ego to alters), reciprocity, and network closure, and several processes of substance use, including differences by gender, usage of other substances (if an adolescent is using a particular type of substance, they are also likely to be using another particular type of substance), and assimilation (influence to use substances by partners who are already using them). 
Finding The Missing Links, 264

These rules are then used to predict the probabilities of connections between actors in the dataset, and the values of target behaviors in the actors. These probabilities are then compared to the observed data to determine the accuracy of the model in describing the generation of the social network. Thus, if a predicted social network closely resembles the observed social network, then the model used to generate the predicted social network can be presumed to be a good one. Next, parameter estimates and standard errors for each of the rules can be examined to determine statistical significance. As long as homophily and influence processes were specified, they will be included in significance analyses.

One particular advantage of this approach comes in its ability to model multi-step processes. For example, in the previously mentioned scenario of two actors who are unconnected and behaviorally dissimilar at time 1, but who have formed a connection and become behaviorally similar to one another in time 2, this model can break the actors' changes into individual events and assess the probabilities of different sequences. Thus, if the model indicates that the actors' behaviors have a low probability of changing, followed by a low probability of them forming a relationship (given their behavior changes); but that there is a high probability of them forming a relationship, followed by a high probability of them changing their behaviors to the present values (given the newly formed connection), the analysis would ultimately indicate that the behavioral similarity of this particular dyad was more likely due to influence than selection. All of these analyses can be performed by the SIENA program (Snijders, Steglich, Schweinberger, \& Huisman, 2005). 
Finding The Missing Links, 265

Although relatively recent, this procedure has already seen use in social networking studies of adolescent substance users (Pearson et al., 2006), adolescent delinquency (Knecht, Snijders, Baerveldt, Steglich, \& Raub, 2010), extra-curricular activities and friendships (Schaefer, Simpkins, Vest, \& Price, 2011), Facebook users' music preferences (Lewis et al., 2012), viral marketing campaigns (Hinz, Skiera, Barrot, \& Becker, 2011), and collaboration networks (Balland, 2012). Nonetheless, the youth of this method comes with a caveat that not all of the mathematics have been worked out yet. Specifically, the distribution of the estimators remains to be determined, the statistical efficiency of the estimator has yet to be thoroughly proven, and the moment equations which the method depends on can sometimes fail to produce a solution (Franzese, Hayes, \& Kachi, 2014). For these reasons, this method will not be employed in this thesis. 